\title{
The Influence of Porous Media Heterogeneity on Smouldering Remediation
}

Jiahao Wang, The University of Western Ontario

Supervisor: Gerhard, Jason I., The University of Western Ontario

Co-Supervisor: Grant, Gavin P., Savron, a subdivision of Geosyntec Consultants

A thesis submitted in partial fulfillment of the requirements for the Master of Engineering

Science degree in Civil and Environmental Engineering

(C) Jiahao Wang 2020

Follow this and additional works at: https://ir.lib.uwo.ca/etd

Part of the Environmental Engineering Commons

\section{Recommended Citation}

Wang, Jiahao, "The Influence of Porous Media Heterogeneity on Smouldering Remediation" (2020). Electronic Thesis and Dissertation Repository. 6790.

https://ir.lib.uwo.ca/etd/6790

This Dissertation/Thesis is brought to you for free and open access by Scholarship@Western. It has been accepted for inclusion in Electronic Thesis and Dissertation Repository by an authorized administrator of Scholarship@Western. For more information, please contact wlswadmin@uwo.ca. 


\begin{abstract}
Smouldering remediation is a promising technique for destroying organic contaminants in soil. Forced airflow is vital to supporting the smouldering reaction and to propagate it through the contaminated zone. This research focuses on investigating the effects of permeability heterogeneity on smouldering. A series of unique column experiments, combined with numerical model simulations, were conducted. The results suggest that smouldering can successfully propagate through layers in series despite more than a 1000:1 permeability contrast. However, extinction can occur in the finer layer when smouldering propagates through layers in parallel with a permeability ratio above $3: 1$. Extinction may occur due to insufficient airflow in the fine layer or due to conductive heat losses from the fine to coarse layers. However, for more complex heterogeneity, smouldering extinction can be eliminated. Overall, this research provides unique insights into managing heterogeneous soils to ensure the successful application of smouldering remediation.
\end{abstract}

\title{
Keywords
}

Smouldering, remediation, permeability, heterogeneity, airflow, heat transfer 


\section{Summary for Lay Audience}

Industrial processes have led to pollution of the environment, including soils and groundwater near former factories. Hydrocarbons, such as tars and petroleum products, are among the most significant challenges in the field of subsurface cleanup. Smouldering combustion, like glowing red charcoal in a barbeque, is a new approach for the destruction of these pollutants. Injecting air into the soil is needed to support this cleanup technique and most soils occur in layers. Therefore, understanding the factors that affect airflow and smouldering patterns in layered soils will help improve the smouldering cleanup of these sites.

A series of smouldering column experiments, combined with computer modelling, were conducted to study the impact of soil layering. The results show how most air flows through the more conductive soil layer, and this can make treatment of the finer layers difficult in certain cases. Besides, loss of heat between layers can cause the treatment to fail in some cases. However, in other cases, smouldering can be successful in all layers, despite large differences in soil types. By explaining the key differences between these cases and how they affect smouldering treatment, it is expected that more polluted sites will be successfully cleaned up in the future. 


\section{Co-Authorship}

The thesis was written in accordance with regulations and guidelines for integrated-article format by the Faculty of Graduate and Postdoctoral Studies at the University of Western Ontario. The candidate collected, analyzed and interpreted relevant data in all experiments under the supervision of Dr. Jason I. Gerhard and with input from Dr. Gavin P. Grant.

Chapter 3: The Influence of Porous Media Heterogeneity on Smouldering Remediation

This Chapter will be submitted to a peer-reviewed journal after the thesis has been completed. Proposed authorship: Jiahao Wang, Jason I. Gerhard and Gavin P. Grant.

Contributions:

Jiahao Wang: $\quad$ performed all laboratory experiments, analysis and interpretation of experimental results, conducted numerical simulations and wrote the chapter.

Jason I. Gerhard: initiated research topic, supervised experiments, modified numerical model, assisted in data interpretation, and reviewed and revised the chapter.

Gavin P. Grant: provided guidance on experimental design, assisted in data interpretation, and reviewed and revised the chapter. 


\section{Acknowledgments}

Back to 2017, I would have never thought today I am sitting in the RESTORE office writing a thesis at Western in Canada, 10,000 km far away from my hometown. During these two years with such an amazing group, I have known many great friends who are the most intelligent, enthusiastic and kind people I have ever met, who keep encouraging me to push myself to step out of the comfort zone and grow up in a surprising direction.

Thank you, Zhejiang Zhouhuan Environmental Engineering Design Co., LTD, I would be nowhere without your Postgraduate Scholarship. I cannot thank you enough for your angel support, as you gave me the possibility to pursue my dream.

I must express my deepest gratitude to my supervisor and mentor Dr. Jason Gerhard, who gave me the invaluable opportunity to come Canada to see the world of smouldering, spent countless hours guiding me on how to become a good researcher and to write improved English sentences. Your optimism, open-mindedness and encouragement helped pull me out of the mud every week in our research meetings; I am not sure where I would be without your unlimited support.

To my co-supervisor, Dr. Gavin Grant, I appreciate your great suggestions from your industrial perspective, which really helped move this project full steam ahead. And thank you for your approachability and valuable time from your busy schedule.

I greatly thank Josh and Rebecca for introducing me to the thrilling world of smouldering experimentation and modelling; your enthusiasm and expertise just make everything enjoyable.

Special thanks to my RESTORE group, in particular, Tarek, Yi and Marco, for being honest and thoughtful mentors and showing me that your future is decided by how you live here and now. Thanks, Taryn, for introducing me to live in a great Canadian lifestyle. Thanks, Shuyang, for helping me in Python and sharing with me each night in the office. And I would like to thank Alex for making great cupcakes for our birthdays; you really make RESTORE our home.

I would like to thank Chu, Haoyi, Zhenchao, Zijun, Wenjuan and Zheying for the important company during the grad school adventure who I have shared many laughs and pains.

Finally, I must thank a friend who was my original motivation to come to Canada and has since changed my life forever. 
Dedication

For my parents 


\section{Table of Contents}

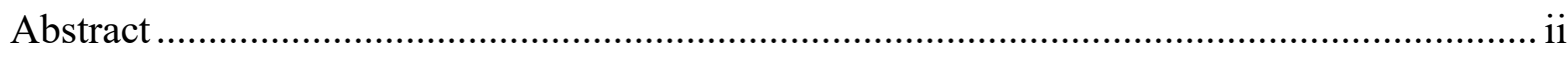

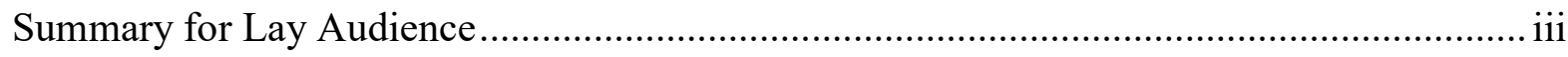

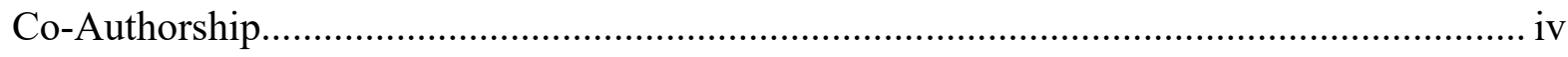

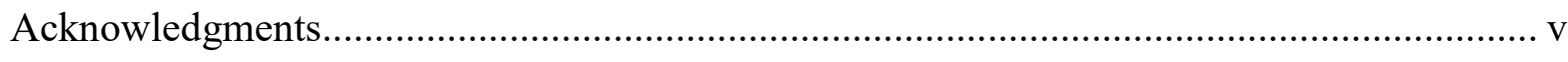

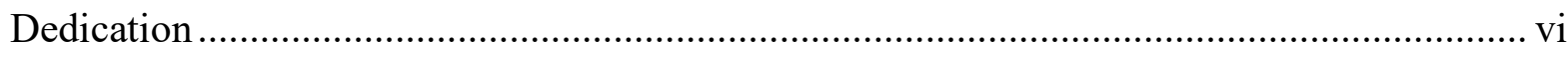

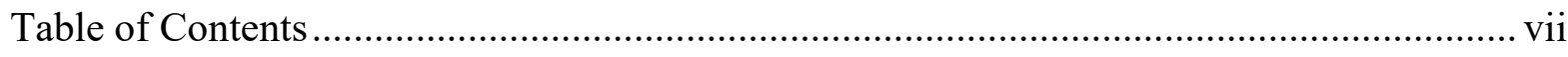

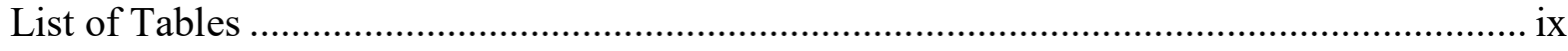

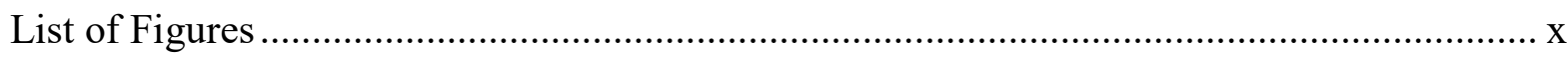

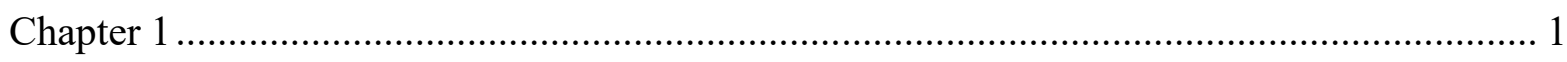

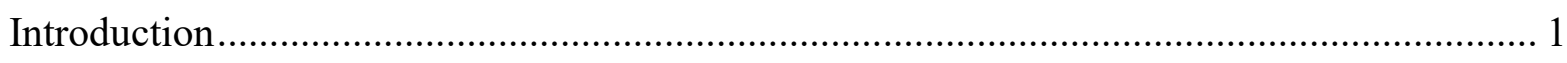

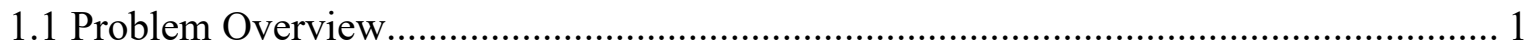

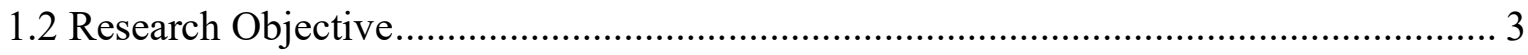

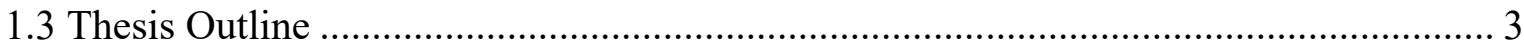

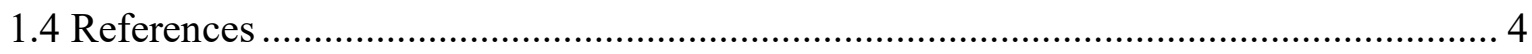

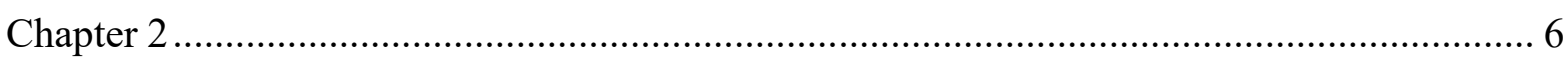

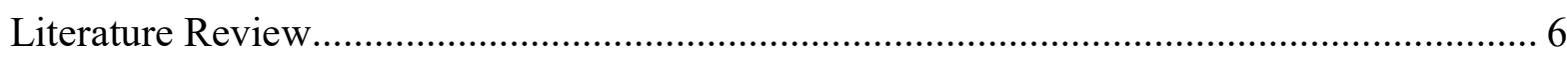

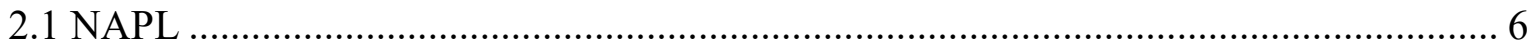

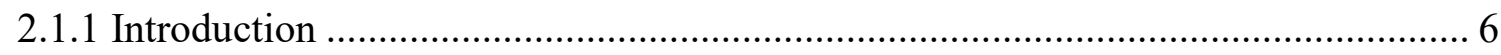

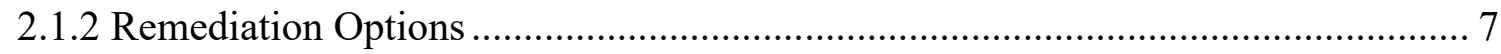

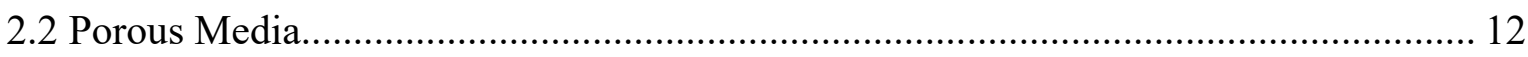

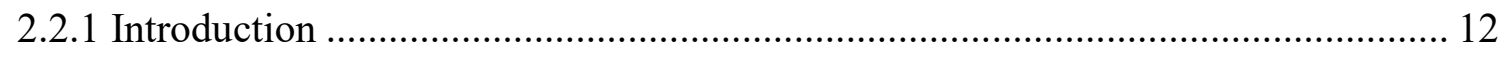

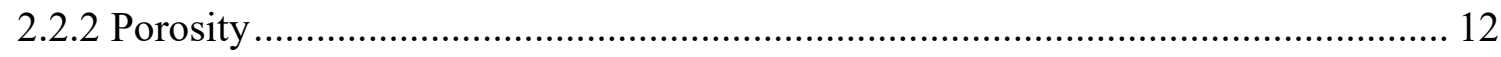

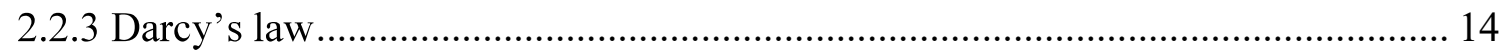

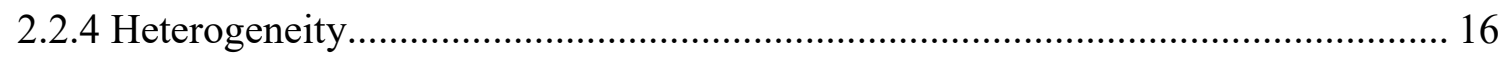

2.2.5 Heat Transfer Through Porous Media .............................................................. 18

2.3 Self-sustaining for Active Remediation (STAR) ................................................ 21

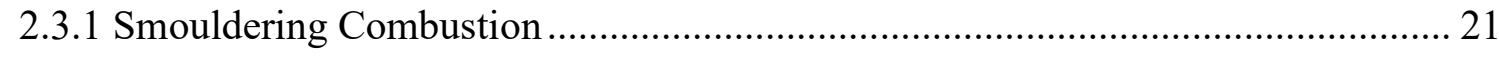

2.3.2 STAR as a NAPL Remediation Technology ................................................. 23

2.3.3 Parameters Affecting Smouldering Performance ............................................. 26

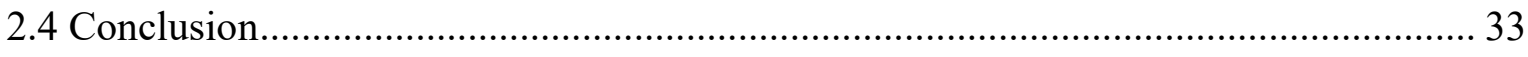

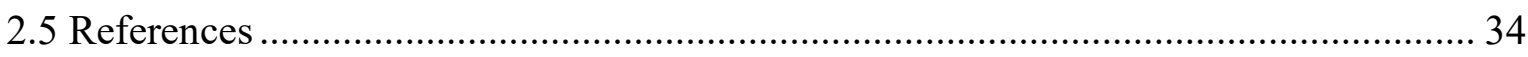


Chapter 3

The Influence of Porous Media Heterogeneity on Smouldering Remediation........................ 41

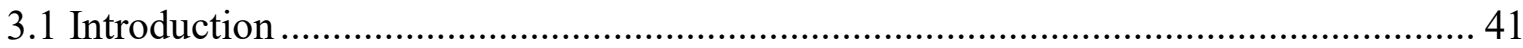

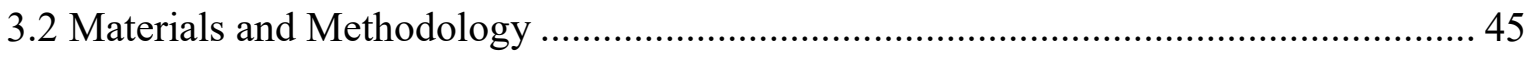

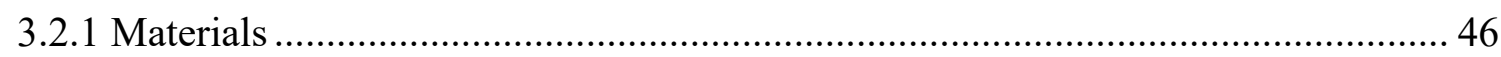

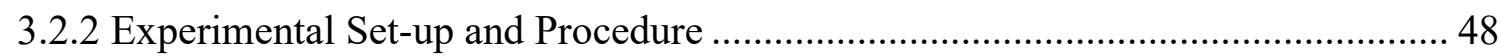

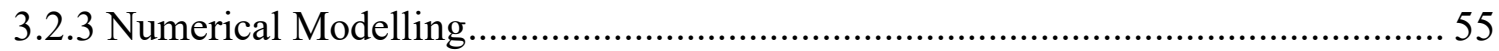

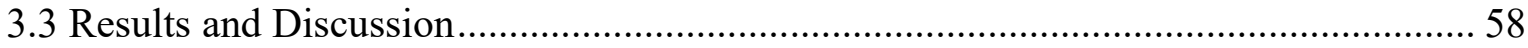

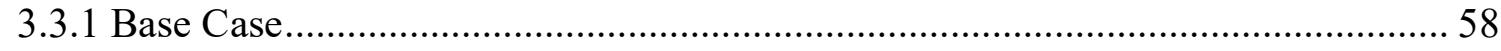

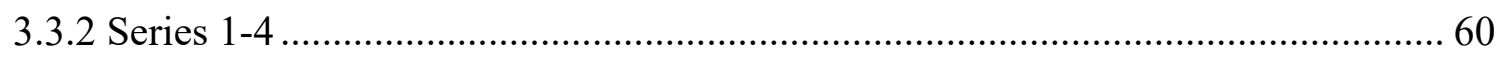

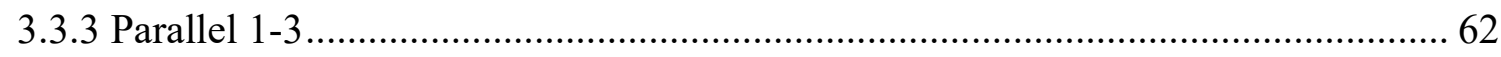

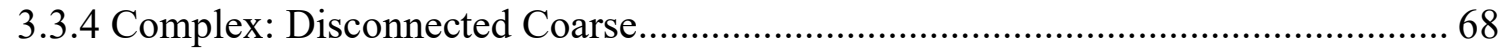

3.3.5 Simulation of Parallel 1-3 and two Complex cases ............................................. 70

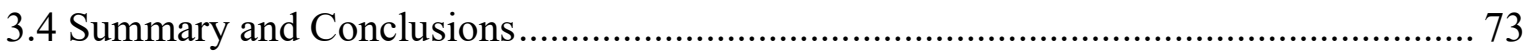

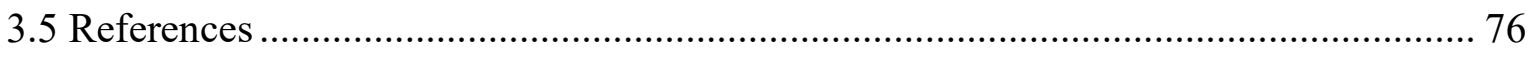

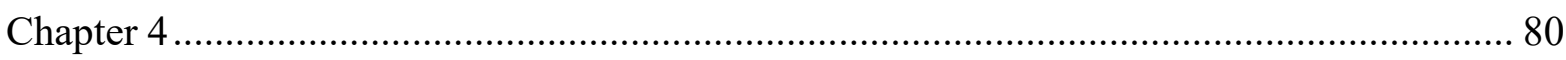

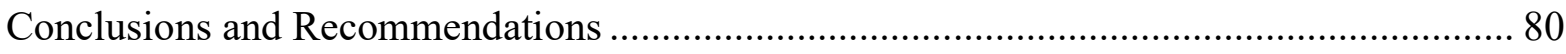

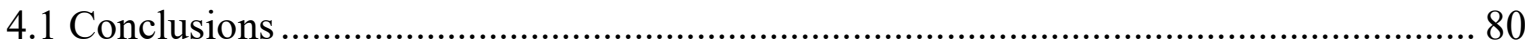

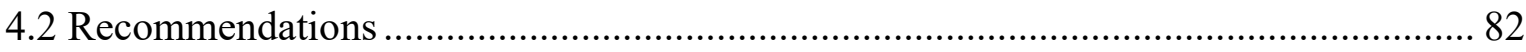

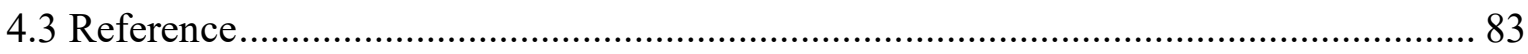

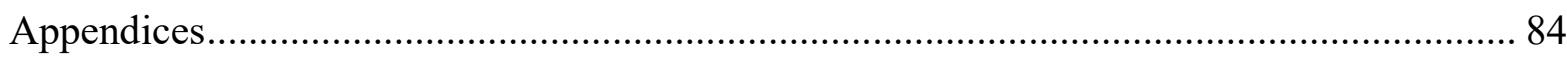

Appendix A: Supporting Information for Sand Properties ............................................... 84

Appendix B: Repeatability of Smouldering Reaction ………………………………..... 88

Appendix C: Experimental Set-up and Excavation …………………………………….... 90

Appendix D: Pressure Changes during Experiments .......................................................... 93

Appendix E: Summary of Principles of ISSM ............................................................... 104

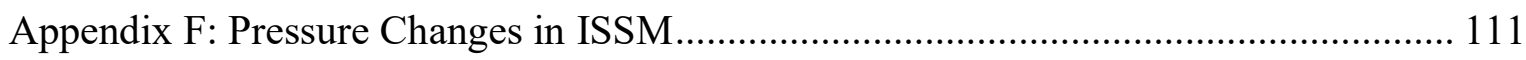

Appendix G: Comparison of ISSM and Experimental Results ....................................... 113

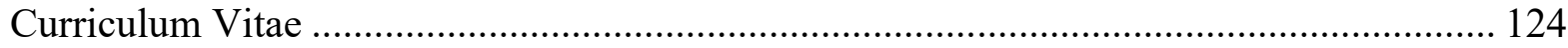




\section{List of Tables}

Table 3.1: Measured Permeability of Seven Sands Used ............................................... 48

Table 3.2: Summary of Smouldering Experiments.......................................................... 49

Table A-1: Permeability and Porosity of Different Porous Media .................................... 85

Table A-2: Repeatable Permeability Test for Sand \#33 ….......................................... 85

Table B-1: Comparison of Upward and Downward Smouldering ...................................... 89 


\section{List of Figures}

Figure 2.1: Conceptual air sparging process diagram (ESTCP, 2001).............................. 8

Figure 2.2: Conceptual schematic of an in-situ permeable reactive barrier (Maitra, 2019).... 9

Figure 2.3: Heater Vacuum Well (Stegemeier and Vinegar, 2001). 10

Figure 2.4: Examples of natural porous materials: (a) beach sand, (b) sandstone and (c) limestone (Nield and Bejan, 2013).

Figure 2.5: The illustration of the representative elementary volume (Nield and Bejan, 2013).

Figure 2.6: Laboratory apparatus to demonstrate Darcy's law (Domenico and Schwartz, 1998).

Figure 2.7: Airflow pattern in coarse uniform sand with fine uniform sand lenses (Reddy and Adams, 2001).

Figure 2.8: Conduction, convection, and radiation heat transfer modes (Bergman et al., 2011).

Figure 2.9: A porous medium. (a) The medium and its properties; (b) Series thermal resistance representation; (c) Parallel resistance representation (Bergman et al., 2011). $\varepsilon$ is the porosity of the medium.

Figure 2.10: A charcoal barbeque is a common example of smouldering from Nielsen (2006).

22

Figure 2.11: Conceptual model of STAR: (a) preheating, (b) ignition, (c) self-sustained smouldering and (d) the end of treatment (Solinger, 2016). 25

Figure 2.12: Schematic representation of the reaction system used in the smouldering of waste (Yermán et al., 2016). 26

Figure 2.13: Peak temperature and smouldering velocity as a function of Darcy flux for smouldering of feces mixed with sand (Yermán et al., 2016).

Figure 2.14: Schematic of the notation used for the propagation of combustion reaction in a two-layered porous medium. $v i$ and $v j$ are the air injection velocities in two layers, $V i$ and $V j$ are the dimensionless front velocities normalized with injection velocities in two layers (Akkutlu and Yortsos, 2005).

Figure 2.15: Simulation (low permeability regions): (a) model domain set-up, (b) distribution of air (vector size range: $0.00-0.430 \mathrm{~m} / \mathrm{s}$ ) and position of the smouldering reaction at $625 \mathrm{~s}$ (10.4 min) following ignition, (c) contour plot depicting the position of the smouldering reaction at $125 \mathrm{~s}(2.1 \mathrm{~min})$ intervals from $\mathrm{t}=0 \mathrm{~s}$ to $1500 \mathrm{~s}$ (25.0 $\mathrm{min})$ (MacPhee et al., 2012).

Figure 3.1: Analogies of the experiments conducted using circuits with (a) resistors in series, and (b) resistors in parallel. R1 is a low resistance (representing the high permeability lens, coarse sand) and R2 is a high resistance (representing the low permeability lens, fine sand). The current flow is analogous to the airflow and the voltage drop is analogous to the pressure drop. 
Figure 3.2: Experimental set-ups for reaction zones in all experiments: (a) Base Case, (b) Series 1-4, (c) Parallel 1-3 and (d) Complex: Disconnected Coarse. The height of each layer is summarized in Table 3.2 .

Figure 3.3: (a) Column A and (b) Column B set up for the smouldering tests.................... 51

Figure 3.4: Illustration of Model set-ups of (a) Parallel 1-3 and (b) Complex: Disconnected Coarse and (c) Complex: Connected Coarse simulations............................................... 57

Figure 3.5: Temperature, pressure, emission and mass loss rate profiles for Base Case....... 59

Figure 3.6: Temperature and pressure profiles for Series 1-4: (a) Series 1, (b) Series 2, (c) Series 3 and (d) Series 4. Black temperature curves are for TCs located in coarse layer while red curves are for TCs located in the fine layer. Note that, while the temperature axes are the same, the pressure axes have different scales in the four figures.

Figure 3.7: Average peak temperature and self-sustained smouldering velocity for each individual layer, plotted with its measured permeability within Series 1-4. 62

Figure 3.8: Temperature, pressure, emission and mass loss rate profiles for Parallel 1....... 63

Figure 3.9: Temperature, pressure, emission and mass loss rate profiles for Parallel 2....... 65

Figure 3.10: Temperature, pressure, emission and mass loss rate profiles for Parallel 3...... 67

Figure 3.11: Temperature, pressure, emission and mass loss rate profiles for Complex: Disconnected Coarse.

Figure 3.12: Air and pressure distributions (red dash-line) in simulations: (a) Parallel 1, (b) Parallel 2, (c) Parallel 3 and (d) Complex: Disconnected Coarse. Each arrow is a velocity vector, with its magnitude expressed by length and direction expressed by orientation. Red dash lines represent pressure contours.

Figure 3.13: Final status of smouldering reaction in simulations: (a) Parallel 1, (b) Parallel 2, (c) Parallel 3 and (d) Complex: Disconnected Coarse. 72

Figure A-1: Particle size distribution curve for silica sand \#12, \#505 and \#106................. 84

Figure A-2: Permeability changes as the mass fraction of fine materials............................. 86

Figure A-3: Porosity changes as the mass fraction of fine materials................................. 86

Figure B-1: Experiments of (a) Column A with Upward Smouldering, (b) Column B with Upward Smouldering and (c) Column B with Downward Smouldering............................ 88

Figure C-1: (a) Smouldering Column A (Base Case, Parallel 1-3 and Complex: Disconnected Coarse) and (b) Column B (Series 1-4). 90

Figure C-2: Loading process for Parallel 1-3 and Complex: Disconnected Coarse, the coarse layer (yellow particles) and fine layer (white particles) were divided by a cardboard sheet, which was taken out before the smouldering. 90 
Figure C-3: Excavation of (a) Parallel 1, (b) Parallel 2, (c) Parallel 3 and (d) Complex: Disconnected Coarse after smouldering treatment, in which white particles were fine materials and small black particles were unburnt GAC left in the porous media................. 92

Figure D-1: Temperature and pressure profiles for Homogeneous 1............................... 93

Figure D-2: Changes of the dynamic viscosity, density and kinematic viscosity of air during smouldering process for Homogeneous 1........................................................................ 96

Figure D-3: Changes of pressure drop and pneumatic conductivity during smouldering

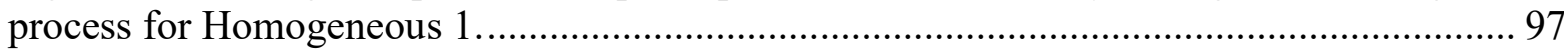

Figure D-4: Temperature and pressure profiles for Homogeneous 2. ............................. 98

Figure D-5: Changes of pressure drop and pneumatic conductivity during smouldering process for Homogeneous 2 .

Figure D-6: Temperature and pressure profiles for Heterogeneous tests: (a) Heterogeneous 1

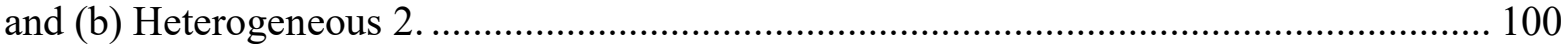

Figure D-7: Temperature and pressure profiles for Heterogeneous tests: (a) Heterogeneous 3

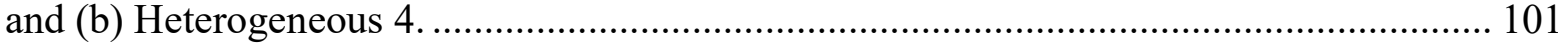

Figure D-8: Temperature and pressure profiles for Heat Test...................................... 102

Figure D-9: Changes of pressures and pneumatic conductivity for Heat Test. .................. 103

Figure E-1: Process flow diagram for the ISSM.......................................................... 104

Figure E-2: Illustration of the front expansion model. ............................................... 107

Figure E-3: Schematic of ellipse dimensions and parameter used in Richards' equations to

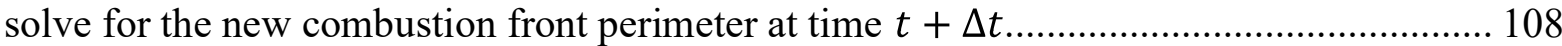

Figure F-1: Air and pressure distributions (red dash-line) in Parallel 1-3 and two Complex cases: (a) Parallel 1, (b) Parallel 2, (c) Parallel 3, (d) Complex: Disconnected Coarse and (e) Complex: Connected Coarse. Each arrow is a velocity vector, with its magnitude expressed by length and direction expressed by orientation. Red dash lines represent pressure contours.

Figure G-1: Comparison of ISSM and Experimental results for Parallel 1 at 3060 seconds.

Figure G-2: Comparison of ISSM and Experimental results for Parallel 1 between 60-30000 seconds.

Figure G-3: Comparison of ISSM and Experimental results for Parallel 2 between 60-30000 seconds.

Figure G-4: Comparison of ISSM and Experimental results for Parallel 3 between 60-30000 seconds.

Figure G-5: Comparison of ISSM and Experimental results for Complex: Disconnected Coarse between 60-30000 seconds. 


\section{Chapter 1}

\section{Introduction}

\subsection{Problem Overview}

Non-aqueous phase liquids (NAPLs) are liquid contaminants that are immiscible with water and can be sources of long-term groundwater pollution in the subsurface because of accidental spills or improper disposal (NRC, 1997; Aggelopoulos et al., 2015). Due to widespread industrial activities in the $20^{\text {th }}$ century and a lack of environmental regulations, sites around the world are contaminated by NAPLs (Gaylor et al., 2000; Gerhard et al., 2007; U.S. EPA, 2009; Kueper et al., 2014). According to the U.S. National Research Council (NRC) review, there are roughly 100, 000 NAPL contaminated sites in the U.S., where complete remediation strategies will not be possible within a reasonable time frame (NRC, 2013; Kueper et al., 2014). This is largely due to the lack of robust and economical remediation technologies available. Most current NAPL remediation technologies are capital intensive, where an assessment from United States Environmental Protection Agency showed that more than \$209 billion dollars will be needed over the next 30 years to remediate around 300, 000 sites in U.S. (U.S. EPA, 2004).

One promising alternative remediation technology is Self-sustaining Treatment for Active Remediation (STAR), which destroys NAPL contaminants embedded in subsurface porous media via smouldering combustion. Smouldering is a slow, controllable, and flameless form of combustion, sustained by the heat generated from heterogeneous oxidation where oxygen directly interacts with the surface of condensed-phase fuels (Ohlemiller, 1985; Switzer et al., 2009). Multiple laboratory experiments and successful field applications have shown that smouldering can be a cost-effective remediation technology for NAPL contaminated sites 
(Pironi et al., 2009, 2011; Switzer et al., 2009, 2014). However, like other remediation technologies, STAR is sensitive to subsurface heterogeneity, especially to permeability differences (i.e., how easily a fluid flows through porous media). For example, Scholes et al. (2015) showed that, during a pilot field test of STAR, preferential smouldering occurred through a highly permeable waste-brick layer.

Currently, few studies have examined the effects of permeability heterogeneity on smouldering propagation. Akkutlu and Yortsos (2005) developed an analytical solution for in situ combustion (an oil recovery technology based on smouldering) in adjacent parallel layers with different permeabilities. Akkutlu and Yortsos (2005) showed conductive heat transfer between two layers, termed "thermal coupling", greatly retarded smouldering propagation velocity in the high permeability layer and slightly increased propagation velocity in the low permeability layer. MacPhee et al. (2012), Hasan et al. (2015), and Solinger et al. (2019) developed a twodimensional, phenomenological in situ smouldering numerical model (ISSM) to approximate the smouldering reaction evolution under various heterogeneous conditions. As observed by Scholes et al. (2015), these studies showed that permeability heterogeneity, from low permeability lenses, can encourage preferential smouldering due to air channelling and leave sections untreated. However, as the ISSM did not simulate temperature evolutions throughout space and time, the contributing temperature effects were not explored (e.g., changing thermophysical properties like density or viscosity with temperature, or thermal coupling) (MacPhee et al., 2012).

Although existing research suggests that intrinsic permeability and heterogeneity may significantly influence smouldering remediation, no studies have examined the effects experimentally or systematically investigated the consequences of permeability heterogeneity. 


\subsection{Research Objective}

The objective of this work is to address this knowledge gap and better understand the role of intrinsic permeability and heterogeneity on smouldering treatment. To achieve this, a laboratory-based experimental study was conducted to explore the practical limits of smouldering treatment with layers of varying permeabilities and orientation (i.e., layers in series, in parallel and combined series and parallel) relative to the direction of smouldering. All smouldering tests used established experimental procedures and data analysis techniques to characterize the smouldering performance, although particular focus in this work on implementing air pressure measurements throughout the layers provided a new understanding of these beneficial datasets. Simulations of some experimental cases with the ISSM provided additional support in understanding the experimental results.

Overall, this work provides unique insights into how intrinsic permeability and heterogeneity affect smouldering propagation. This insight has significant implications towards implementing STAR in real-world field sites, which often exhibit heterogeneity, and in reactor-based applications where the degree of heterogeneity can be controlled. In a broader sense, these results are expected to help improve basic understanding of smouldering science as well as the application of other remediation and porous media technologies associated with heated airflow in layered systems, such as thermal desorption.

\subsection{Thesis Outline}

This thesis is written in an integrated article format in accordance with the guidelines and regulations stipulated by the Faculty of Graduate Studies at the University of Western Ontario. Each chapter in the thesis is described below. 
Chapter 2 is a review of the relevant literature and presents an overview of existing remediation technologies for NAPL contamination. Relevant mass and heat transfer through porous media are reviewed. Furthermore, an introduction to smouldering combustion and STAR is presented, in which key parameters that affect smouldering are reviewed.

Chapter 3 presents all the results from the laboratory experiments and ISSM simulations that investigated the effect of intrinsic permeability and heterogeneity on smouldering propagation. This chapter is written in a manuscript format for future submission to a peerreviewed journal.

Chapter 4 summarizes all key observations, conclusions and limitations in this study, including recommendations for future research.

Appendices provide supplemental information, which are referenced throughout the thesis.

\subsection{References}

Aggelopoulos, C. A., Tsakiroglou, C. D., Ognier, S., \& Cavadias, S. (2015). Non-aqueous phase liquid-contaminated soil remediation by ex situ dielectric barrier discharge plasma. International Journal of Environmental Science and Technology, 12(3), 10111020.

Akkutlu, I. Y., \& Yortsos, Y. C. (2005). The Effect of Heterogeneity on In-Situ

Combustion: Propagation of Combustion Fronts in Layered Porous Media. SPE Journal, 10(04), 394-404.

Gaylor, D. W., Culp, S. J., Goldstein, L. S., \& Beland, F. A. (2000). Cancer Risk Estimation for Mixtures of Coal Tars and Benzo(a)pyrene. Risk Anal, 20(1), 81.

Gerhard, J. I., Pang, T., \& Kueper, B. H. (2007). Time Scales of DNAPL Migration in Sandy Aquifers Examined via Numerical Simulation. Groundwater, 45(2), 147.

Hasan, T., Gerhard, J. I., Hadden, R., \& Rein, G. (2015). Self-sustaining smouldering combustion of coal tar for the remediation of contaminated sand: Two-dimensional experiments and computational simulations. Fuel, 150, 288-297.

Kueper, B. H., Stroo, H.F., Vogel, C. M., \& Ward, C. H. (2014). Chlorinated Solvent Source Zone Remediation. New York: Springer. 713 p. 
MacPhee, S. L., Gerhard, J. I., \& Rein, G. (2012). A novel method for simulating smoldering propagation and its application to STAR (Self-sustaining Treatment for Active Remediation). Environmental Modelling \& Software, 31, 84-98.

NRC (National Research Council). (1997). Innovations in ground water and soil cleanupFrom concept to commercialization. Washington, D.C: National Academies Press. $292 \mathrm{p}$.

NRC (National Research Council). (2013). Alternatives for managing the nation's complex contaminated groundwater sites. Washington, DC: The National Academies Press. 422 p.

Ohlemiller, T. J. (1985). Modeling of smoldering combustion propagation. Progress in Energy and Combustion Science, 11(4), 277-310.

Pironi, P., Switzer, C., Rein, G., Fuentes, A., Gerhard, J. I., \& Torero, J. L. (2009). Smallscale forward smouldering experiments for remediation of coal tar in inert media. Proceedings of the Combustion Institute, 32(2), 1957-1964.

Pironi, P., Switzer, C., Gerhard, J. I., Rein, G., \& Torero, J. L. (2011). Self-sustaining smoldering combustion for NAPL remediation: laboratory evaluation of process sensitivity to key parameters. Environmental Science \& Technology, 45(7), 29802986.

Scholes, G. C., Gerhard, J.I., Grant, G.P., Major, D.W., Vidumsky, J.E., Switzer, C., \& Torero, J.L. (2015). Smoldering remediation of coal-tar-contaminated soil: pilot field tests of STAR. Environmental Science \& Technology. 49, 14334-14342.

Solinger, R., Grant, G.P., Scholes, G.C., Murray, C., \& Gerhard, J.I. (2019). STARx hottpad for smouldering treatment of waste oil sludge: proof of concept and sensitivity to key design parameters. Accepted for publication in Waste Management \& Research.

Switzer, C., Pironi, P., Gerhard, J. I., Rein, G., \& Torero, J. L. (2009). Self-sustaining smoldering combustion: A novel remediation process for non-aqueous-phase liquids in porous media. Environmental Science \& Technology, 43(15), 5871-5877.

Switzer, C., Pironi, P., Gerhard, J. I., Rein, G., \& Torero, J. L. (2014). Volumetric scale-up of smouldering remediation of contaminated materials. Journal of Hazardous Materials, 268, 51-60.

U.S. EPA (U.S. Environmental Protection Agency). (2004). Cleaning up the nation's waste sites: Markets and technology trends. EPA-542-R-04-015. Office of Solid Waste and Emergency Response. 338 p.

U.S. EPA (U.S. Environmental Protection Agency). (2009). Assessment and Delineation of DNAPL Source Zones at Hazardous Waste Sites. EPA-600-R-09-119. National Risk Management Research Laboratory. 20 p. 


\section{Chapter 2}

\section{Literature Review}

\subsection{NAPL}

\subsubsection{Introduction}

NAPLs are liquid contaminants which exist as a separate, immiscible phase when in contact with water (U.S. EPA, 1995). Common examples of NAPLs include petroleum hydrocarbons, chlorinated solvents, pesticides, herbicides, creosote, coal tar and polychlorinated biphenyls (Mercer and Cohen, 1990). Within the last 70 years, these compounds were heavily used throughout the industrialized world (Kueper et al., 2014). However, due to a lack of understanding regarding the fate and transport of these contaminants in the subsurface, along with underestimating the risks associated with their toxicity, environmental regulations significantly lagged behind their production, use, and disposal in the industry (Pankow and Cherry, 1996). As a result, improper disposal and accidental spills have led to widespread NAPL contamination at hazardous waste sites around the world. These NAPL contaminated sites pose a serious threat to humans and surrounding natural ecosystems (Pankow and Cherry, 1996).

Unfortunately, once NAPLs enter the subsurface, they are very challenging to remediate. These remediation challenges are due to the multiphase interactions between NAPLs, air, water and soil, and subsurface heterogeneity, which explain why traditional remediation technologies and strategies are capital-intensive and time-consuming. For example, thick confining layers (e.g., clay) and bedrock formations with low permeability may initially be a sink for contaminants, where dissolved NAPL will diffuse into these formations. However, as remediation progresses and the surrounding water concentrations decrease, 
the dissolved NAPL that first diffused in these formations may back diffuse into the surrounding groundwater. This back diffusion may significantly lengthen the time to complete remediation and is a major challenge for remediation practitioners (Coutelieris and Delgado, 2012). Therefore, it is challenging to remediate a NAPL contaminated site completely in a cost-effective manner, particularly at large-scale sites (Kueper et al., 2014).

\subsubsection{Remediation Options}

With the improvement of environmental legislation since the 1970s (especially with the Superfund legislation (Hird, 1993)), there was a surge of activities towards improving disposal practices at operating industrial facilities, and remediation of previously contaminated sites (Rosenbaum, 2019). This led to a strong need for innovative NAPL remediation technologies. According to U.S. EPA (2002), over a 17-year period from 1982 through 1999, more than 2200 Records of Decision (RODs) had been signed for 1451 Superfund sites, including 787 contaminated groundwater sites. As for the remediation strategies at these sites, U.S. EPA (2002) showed that pump and treat (P\&T) had been the most popular remediation strategy but decreased from $92 \%$ in 1986 to $30 \%$ in 1999, whereas alternative methods, such as monitored natural attenuation, increased from $8 \%$ in 1986 to $44 \%$ in 1998 . Likewise, other in situ treatment technologies including air sparging and bioremediation increased from 9\% (1995) to 35\% (1999) (U.S. EPA, 2002). These trends showed that even though P\&T was still the most popular remediation strategy, other alternative technologies were gaining widespread acceptance and are worthwhile to review. 


\section{(1) Air Sparging}

Air sparging is an in situ remediation technology that is best suited for volatile organic compounds, which are commonly found adsorbed to soils and dissolved in the groundwater, e.g., lower chlorinated ethenes (trichloroethylene, dichloroethene) and lower chlorinated ethanes (trichloroacetic acid and dichloroacetic acid) (ESTCP, 2001). Air is bubbled into the subsurface, below the water table within the NAPL plume, and the contaminant mass is transferred from the dissolved and adsorbed phase to their vapour phase (U.S. EPA, 2017). Because of buoyancy, the air bubbles move upward towards the vadose zone, where the air is usually collected for central processing, typically with a soil vapour extraction (SVE) system (Figure 2.1). When air sparging is combined with SVE, the SVE system will provide a negative pressure zone above the target zone through soil vapour extraction wells, extracting the vapour phase of contaminants as well as controlling the movement of vapour plume.

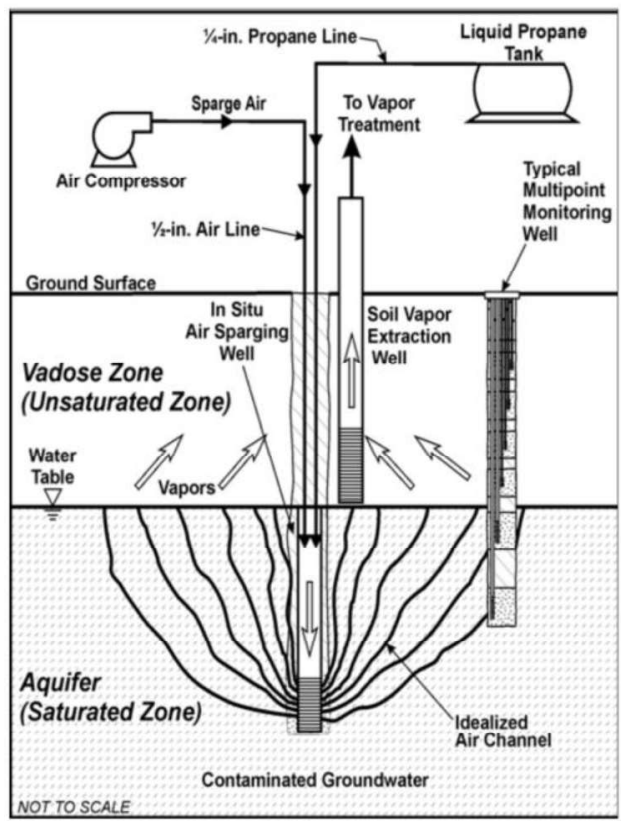

Figure 2.1: Conceptual air sparging process diagram (ESTCP, 2001). 
(2) Permeable Reactive Barrier

A permeable reactive barrier (PRB) is an engineered zone consisting of reactive media, which is placed in the aquifer flow path to passively remediate contaminants as the groundwater flows through it, see Figure 2.2 (Naftz et al., 2002).

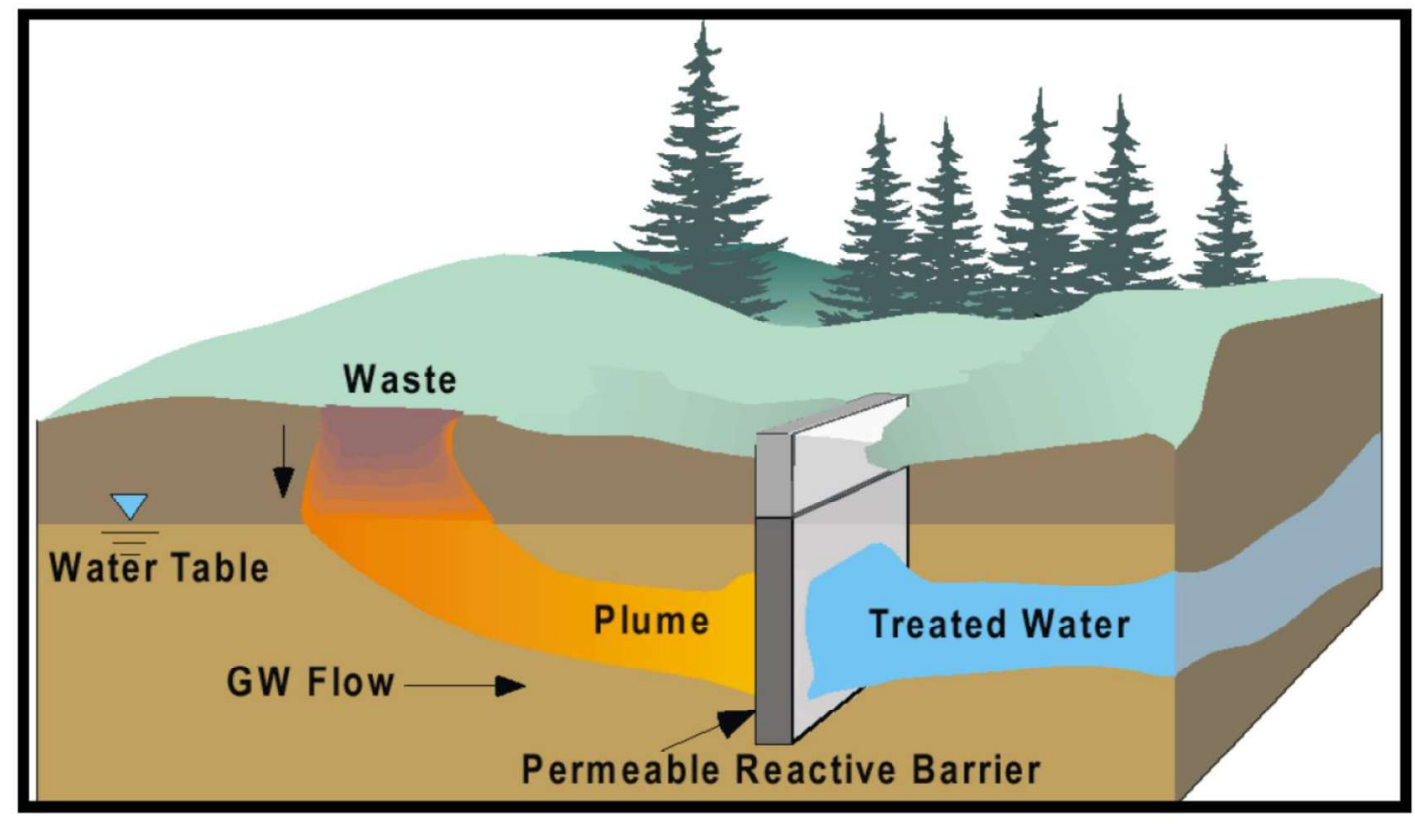

Figure 2.2: Conceptual schematic of an in-situ permeable reactive barrier (Maitra, 2019).

Besides the physical processes in the barrier, including precipitation and sorption, researchers reported that biological reactions are important mechanisms of contaminant removal in PRBs since contaminants directly contact with media (Atlas and Philp, 2005; Thiruvenkatachari et al., 2008; Obiri-Nyarko et al., 2014). Various bio-enhancement methods have been proposed to improve the bio-remediation activities in PRB (Maitra, 2019). For example, reactive barriers containing oxygen-releasing compounds have been applied successfully to stimulate aerobic bio-degradation of monoaromatic hydrocarbons such as benzene, toluene, ethylbenzene, and xylenes (BTEX) (Scherer et al., 2000). In short, 
PRB can be an efficient in situ remediation technology, which is able to remove both organic and inorganic contaminants from the groundwater, including heavy metals and chlorinated compounds.

\section{(3) Thermal Conduction Heating}

Thermal conduction heating is a soil remediation process where heat and vacuum are applied simultaneously, as shown in Figure 2.3 (Stegemeier and Vinegar, 2001). During the process, energy radiating from thermal wells diffuses into the adjacent treatment area, typically to peak temperatures between $800{ }^{\circ} \mathrm{C}$ and $900{ }^{\circ} \mathrm{C}$. As soil is heated, the contaminants in the soil are volatilized or destroyed by oxidation or pyrolysis reactions. The emissions (e.g., vaporized water, volatile matter, hydrocarbon) are drawn out via the vacuum inside the heater and collected for further treatment. In practice, most contaminants are destroyed at high temperatures before they reach the surface area (Stegemeier and Vinegar, 2001).

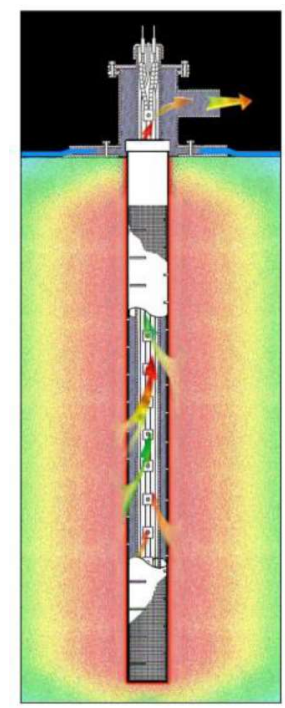

Figure 2.3: Heater Vacuum Well (Stegemeier and Vinegar, 2001). 
In summary, all remediation technologies have unique strengths for various site conditions. For example, air sparging is a good technology to remove light non-aqueous phase liquids (LNAPLs), as it is easy to install in the field and requires a short treatment period for LNAPLs, compared to other remediation technologies (U.S. EPA, 2017). PRBs are well suited to handle complicated plume structures by stretching the PRB over these complexities (U.S. EPA, 2000; Maitra, 2019). Thermal conduction heating is a fast treatment technology with a high removal rate for most volatile and semi-volatile organic pollutants, and can be enhanced by injecting air near the heated area, that is, combined with air sparging (Stegemeier and Vinegar, 2001). However, each technology also has unique limitations. Air sparging is hard to apply in the confined aquifers or stratified soils because of the high pressures required. Furthermore, air sparing may also cause unexpected migration of contaminants (U.S. EPA, 2017). As PRBs are intended to treat plumes, they are not well suited to treat insoluble or immobile contaminants. Additionally, the efficiency of PRBs depends on the groundwater flow rate. Therefore, areas with low flow, e.g., due to minimal water head changes or low soil permeability, require a long time to treat a slowly travelling plume (Maitra, 2019). Because thermal conduction heating needs to heat the whole target site to a certain temperature, it is limited by the thermophysical properties of soils, weather conditions, and it may require a high density of wells and a large energy input.

Altogether, even though there are promising remediation technologies for specific site conditions, no technology can deal with all contaminants at all sites. There are no silver bullets. Therefore, effective remediation relies on a strong understanding of the 
contaminant chemistry and subsurface conditions to best choose (possibly multiple) suitable remediation technologies (U.S. EPA, 2018).

\subsection{Porous Media}

\subsubsection{Introduction}

A porous medium is defined as a material that consists of a solid matrix with an interconnected network of pores (Nield and Bejan, 2013). It is widely accepted that most materials in the real world are, to some extent, porous, so it is difficult to find a perfectly "non-porous" material (Coutelieris and Delgado, 2012). Common examples of natural porous media are beach sand, sandstone, and limestone. The characteristics of porous media are mainly determined by the structure of the pores, in which the interconnectedness of the pores allows single or multiple fluids to pass through the medium.
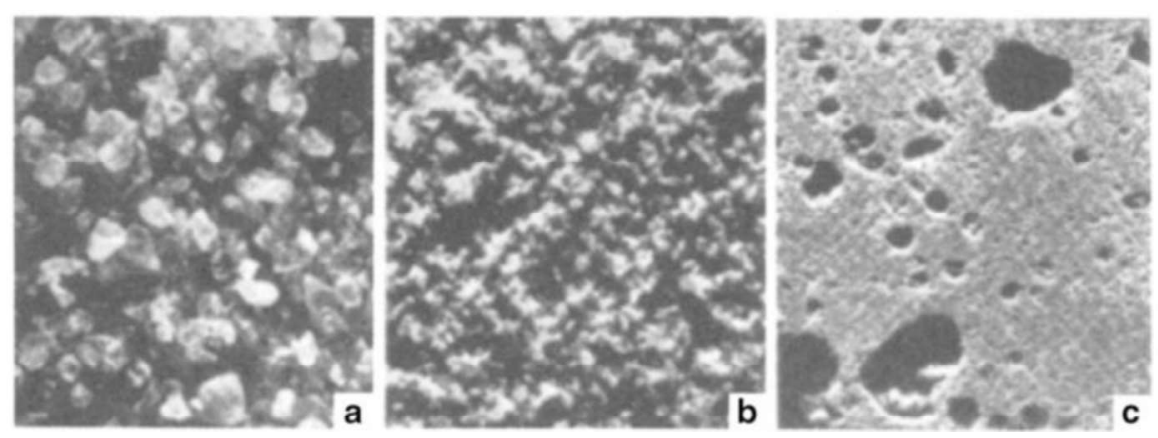

Figure 2.4: Examples of natural porous materials: (a) beach sand, (b) sandstone and (c) limestone (Nield and Bejan, 2013).

\subsubsection{Porosity}

As the flow and other transport processed through a porous medium are complicated at the pore scale, it is often convenient to average the medium properties over a larger scale containing many pores either following the spatial or statistical approach. In the spatial 
approach, a representative elementary volume (REV) is used as a macroscopic scale to describe meaningful properties over a sufficiently large volume, which is widely used (Domenico and Schwartz, 1998). In the statistical approach, an ensemble of possible pore structures that are macroscopically equivalent are averaged. However, this approach does not maintain the heterogeneous characteristics, as the differences are averaged out (Nield and Bejan, 2013).

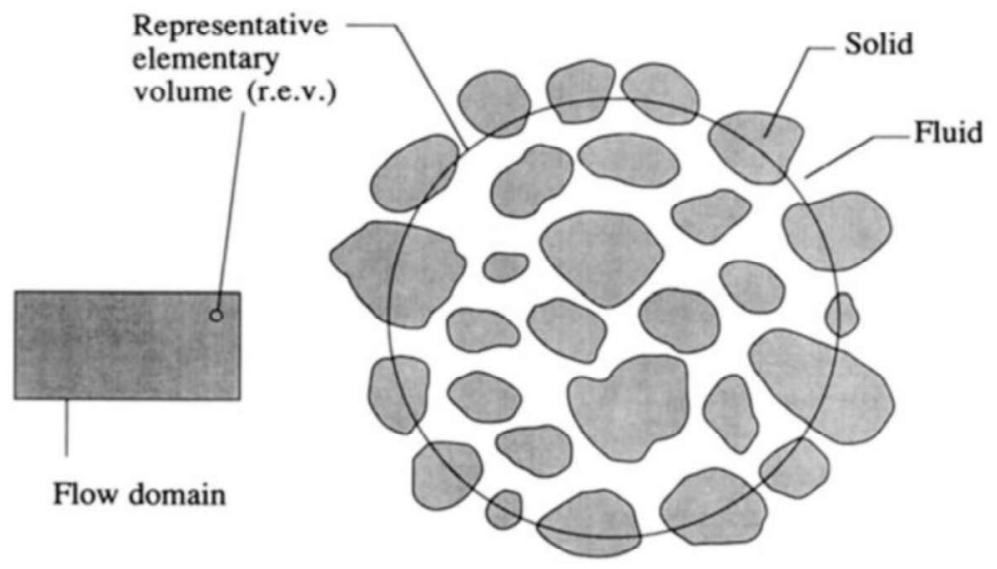

Figure 2.5: The illustration of the representative elementary volume (Nield and Bejan, 2013).

The porosity $\emptyset$ is a REV scale parameter that defines as the volume fraction of pores; thus, $1-\emptyset$ is the volume fraction of the solid matrix. Usually, it is assumed that all pores are interconnected inside the porous media; however, it is more common to find isolated and disconnected pores. An "effective porosity" can be defined based on the fraction of connected pores (Nield and Bejan, 2013). In a natural porous medium, the porosity rarely exceeds 0.6 , for example, the porosity of silica sand can vary from 0.2 (poorly sorted) to 0.5 (well sorted). However, engineered porous media vary much more widely depending on the application, such as metallic foams with the porosity around 1. In addition, the nonuniformity in the porosity near the boundaries (e.g., confining solid or free surface) can 
play an important role in the fluid transport rate through the porous media because of the different particle arrangement near the boundaries compared to the bulk media. When the boundary is a confining solid, the larger porosities close to the surface reduce the local resistance to fluid flow, increasing the local fluid velocity within this area, i.e., channelling (Kaviany, 1991).

\subsubsection{Darcy's law}

The process of fluid flow through porous media is of great interest to hydrogeologists and geotechnical engineers (Whitaker, 1986). The first one-dimensional empirical equation was experimentally determined by Darcy in 1856, named as Darcy's law, which generally describes groundwater flow in most natural settings, though it breaks down at large Reynold's numbers, e.g., in karst formations with large pores or high velocity zones near well screens (Schwartz and Zhang, 2003).

As shown in Figure 2.6, an energy gradient, as observed as the different heights the fluid rises at two points (piezometric head), will drive flow, where the amount of energy lost is due to friction as the fluid flows through the porous media (Schwartz and Zhang, 2003). From 35 seminal experiments, Darcy (1865) found that the flow rate through a porous medium was proportional to the piezometric head difference, expressed mathematically as:

$$
Q=K A \frac{h_{1}-h_{2}}{L} \text { or } q=-K \frac{d h}{d L}
$$

here $Q$ is volumetric flow rate $\left(\mathrm{m}^{3} / \mathrm{s}\right), K$ is hydraulic conductivity $(\mathrm{m} / \mathrm{s}), A$ is crosssectional area $\left(\mathrm{m}^{2}\right), h_{1}-h_{2}$ is the difference piezometric head (m), $L$ is the length of the sand column (m), $q$ is fluid flux $(\mathrm{m} / \mathrm{s}), d h / d l$ is the piezometric head gradient within a certain length of the sand sample. Hydraulic conductivity is also a REV scale parameter, 
which refers to the ability of a medium to transmit fluids and is dependent on properties of the fluid (i.e., density and viscosity) and the medium (permeability).

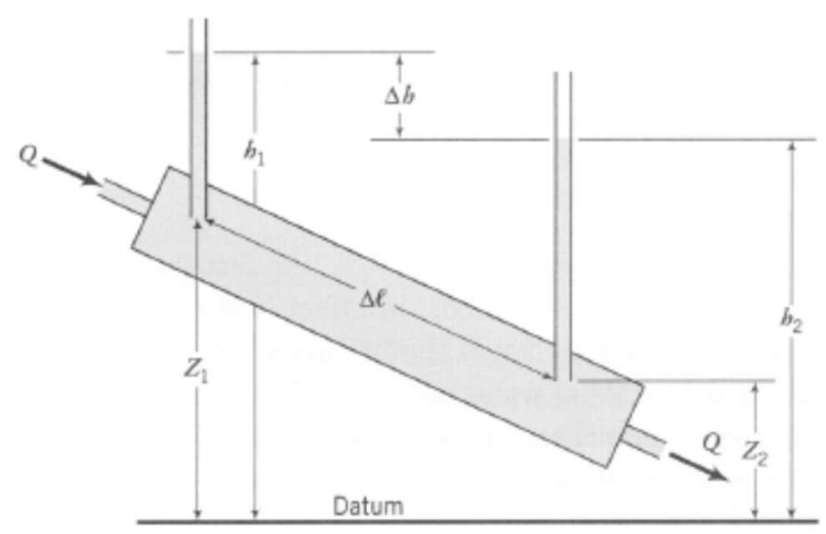

Figure 2.6: Laboratory apparatus to demonstrate Darcy's law (Domenico and Schwartz, 1998).

Intrinsic permeability is embedded within hydraulic conductivity, revealing the effect of porous media structure, e.g., interconnectedness of pores, size of pores, on the fluid flow. The relationship between hydraulic conductivity and intrinsic permeability is defined by (Schwartz and Zhang, 2003):

$$
K=\frac{k \rho g}{\mu}
$$

here $k$ is intrinsic permeability $\left(\mathrm{m}^{2}\right)$ of the porous medium, $\rho$ and $\mu$ are density $\left(\mathrm{kg} / \mathrm{m}^{3}\right)$ and viscosity $(\mathrm{kg} / \mathrm{m} / \mathrm{s})$ of the fluid, respectively, and $g$ is gravitational acceleration $\left(\mathrm{m} / \mathrm{s}^{2}\right)$.

The Kozeny-Carman equation, derived by Kozeny (1927) and Carman (1937,1956), highlights the relationship between permeability, and porosity and particle size of the material, which assumes a porous medium is comprised of perfectly round spheres with uniform diameters: 


$$
k=\frac{\emptyset^{3}}{36 \kappa(1-\varnothing)^{2}} d^{2}
$$

here $\kappa$ is the $\mathrm{KC}$ constant, $\emptyset$ is the porosity of the material and $d$ is the mean diameter for hypothetical spherical solid particles $(\mathrm{m})$ (Xu and $\mathrm{Yu}, 2008)$. Five is a typical value widely used for $\kappa$, whereas experiments, numerical calculation and numerous theoretical models indicate that the Kozeny-Carman equation does not lead to a constant $\kappa$, which is affected by the porosity, microstructure of pores and capillaries (Happel and Brenner, 1986; Kaviany, 1991).

\subsubsection{Heterogeneity}

The classical definition in Equation 2 considers that the porous medium has uniform properties in a given direction, i.e., isotropic, and is the same from point to point, i.e., homogeneous. In real life, most porous media do not satisfy these conditions and are, to some degree, heterogeneous and anisotropic (Schwartz and Zhang, 2003). Based on the data compiled by Scheidegger (1974), Bejan and Lage (1991), it showed permeability difference of common porous materials can easily be up to four orders of magnitude (e.g., $10^{-10} \mathrm{~m}^{2}$ for sand and $10^{-14} \mathrm{~m}^{2}$ for silica powder). Research on heterogeneity in natural geological systems has shown substantial soil variabilities even at the same site. For example, Pryor (1973) found that samples from the same river bar had permeabilities ranging from $2.5 \times 10^{-15} \mathrm{~m}^{2}$ to more than $1 \times 10^{-10} \mathrm{~m}^{2}$.

The influence of permeability heterogeneity is a major challenge for mapping contamination, as it governs the transport behaviour of pollutants. In aquifers, contaminant plume distributions are often difficult to predict in highly heterogeneous formations due to the complex advection and dispersion driven by heterogeneous soil 
properties (Levy and Berkowitz, 2003; Berkowitz et al., 2006; Janković et al., 2006; Hunt et al., 2011; Fiori et al., 2013). As a specific example, Page et al. (2007) studied the role of heterogeneity on mass flux from DNAPL source zones in a series of intermediate-scale tank experiments, and it was observed that DNAPL preferentially migrated between the sand lenses of greatest permeability by pore-scale fingering.

Aside from contaminant transport, permeability heterogeneity is also critical for remediation strategies, especially those that involve the injection of a fluid, such as chemical oxidation and air sparging. During the chemical oxidation process, oxidants (e.g., potassium permanganate) are injected underground into contaminated areas. During injection, the permeability heterogeneity effectively governs where the oxidants are transported, resulting in an intensive dose in high permeability areas, whereas little treatment in the low permeability areas (Seol et al., 2003). The problem is very similar for air sparging, as another fluid, i.e., air, is injected into the subsurface below the lowest known point of contamination. Reddy and Adams (2001) found that when the permeability ratio $\left(k_{\text {coarse }} / k_{\text {fine }}\right)$ between two adjoining layers or lenses is greater than 10:1, such as fine materials embedded in coarse sand, air bypasses the lower permeability areas, resulting in limited contaminant removal in the fine layers (Figure 2.7). In this scenario, contaminant removal from the low permeability areas will be driven by diffusion due to the concentration gradient induced by air sparging, which is slow and therefore lengthens the treatment time and increases remediation cost (Reddy and Adams, 2001). 


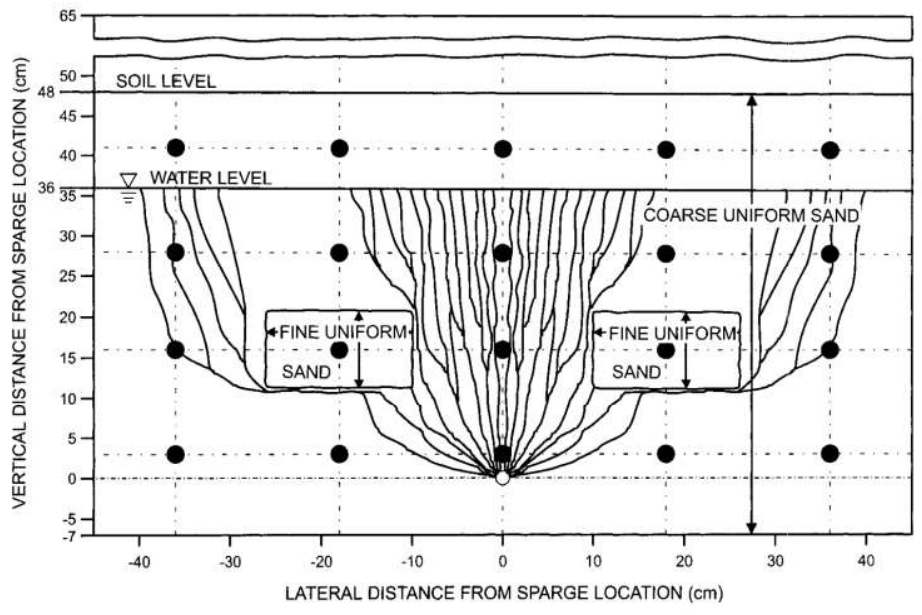

Figure 2.7: Airflow pattern in coarse uniform sand with fine uniform sand lenses (Reddy and Adams, 2001).

\subsubsection{Heat Transfer Through Porous Media}

Heat transfer occurs anywhere that a temperature difference exists, and occurs as conduction, convection and radiation (Figure 2.8).

\begin{tabular}{|l|l|l|}
\hline $\begin{array}{l}\text { Conduction through a solid } \\
\text { or a stationary fluid }\end{array}$ & $\begin{array}{l}\text { Convection from a surface } \\
\text { to a moving fluid }\end{array}$ & $\begin{array}{l}\text { Net radiation heat exchange } \\
\text { between two surfaces }\end{array}$ \\
\hline & & Moving fluid, $T_{\infty}$ \\
\hline
\end{tabular}

Figure 2.8: Conduction, convection, and radiation heat transfer modes (Bergman et al., 2011).

Conduction refers to the heat transfer due to molecular collisions and energy is transferred from the more energetic to the less energetic particles (Bergman et al., 2011). The rate of conduction heat transfer is expressed as:

$$
q_{x}^{\prime \prime}=-k \frac{d T}{d x}
$$


here $q_{x}^{\prime \prime}\left(\mathrm{W} / \mathrm{m}^{2}\right)$ is the heat flux (heat transfer rate) in the $\mathrm{x}$-direction per unit area perpendicular to the direction of heat transfer, which is proportional to the temperature gradient, $d T / d x$, and $k$ is the thermal conductivity $(\mathrm{W} / \mathrm{m} / \mathrm{K})$ that is a characteristic of the material. Research shows that the thermal conductivities of dry soils are relatively similar, and generally only differ by a factor of two (Stegemeier and Vinegar, 2001; Zanoni et al., 2017). However, like all materials, the thermal conductivity does change with temperature, where Zanoni et al. (2017) showed that the thermal conductivity of coarse grain sand particles linearly increased with temperature from $36^{\circ} \mathrm{C}$ to $265^{\circ} \mathrm{C}$.

Convection heat transfer occurs between a fluid in motion and a bounding surface. In addition to the diffusion of energy from colliding molecules, heat is also transferred by the bulk, or macroscopic, motion of the fluid (Bergman et al., 2011). Most of the heat transfer occurs near the fluid-surface interface in the heat-transfer boundary layer (Bergman et al., 2011). Based on the nature of flow, convection heat transfer is generally classified into two types: (1) forced convection when the flow is caused by external means (e.g., a fan, a pump); (2) natural convection when the buoyancy effect drives convective flows. The appropriate rate equation of convection is expressed as:

$$
q^{\prime \prime}=h\left(T_{s}-T_{\infty}\right)
$$

where $q^{\prime \prime}$ is the convection heat flux $\left(\mathrm{W} / \mathrm{m}^{2}\right)$, proportional to the temperature difference between the surface area and fluid, $T_{s}$ and $T_{\infty}$, and $h\left(\mathrm{~W} / \mathrm{m}^{2} / \mathrm{K}\right)$ is the convection heat transfer coefficient, which depends on the conditions in the boundary layer (e.g., the nature of the fluid motion). The convection heat transfer coefficient increases as the local fluid velocity increases (ASHRAE, 1997; Brecht et al., 2005). 
Radiation is energy emitted by matter that is at nonzero temperature, which is transported by electromagnetic waves (Bergman et al., 2011).

In porous media, heat transfer is more complicated as it occurs as between multiple phases (e.g., between water and sand in a saturated medium) (Figure 2.9a). If the temperature difference between phases is small, then the material is in local thermal equilibrium and can estimate the heat transfer rate:

$$
q_{x}=\frac{k_{e f f} A}{L}\left(T_{1}-T_{2}\right)
$$

where $k_{\text {eff }}(\mathrm{W} / \mathrm{m} / \mathrm{K})$ is an effective thermal conductivity, considering both thermal conductivities of the fluid $k_{f}$ and solid porous medium $k_{s}$. The value of effective thermal conductivity for a porous medium can be bracketed by considering the composite walls in Figure 2.9b and 2.9c (Bergman et al., 2011; Nield and Bejan, 2013). Equation 6 is only valid for the estimation of heat transfer rate in the porous medium if convection as well as radiation heat transfer within the porous medium are negligible. Radiation heat transfer inside porous media was incorporated using the diffusion approximation by Dosanjh et al. (1987). 


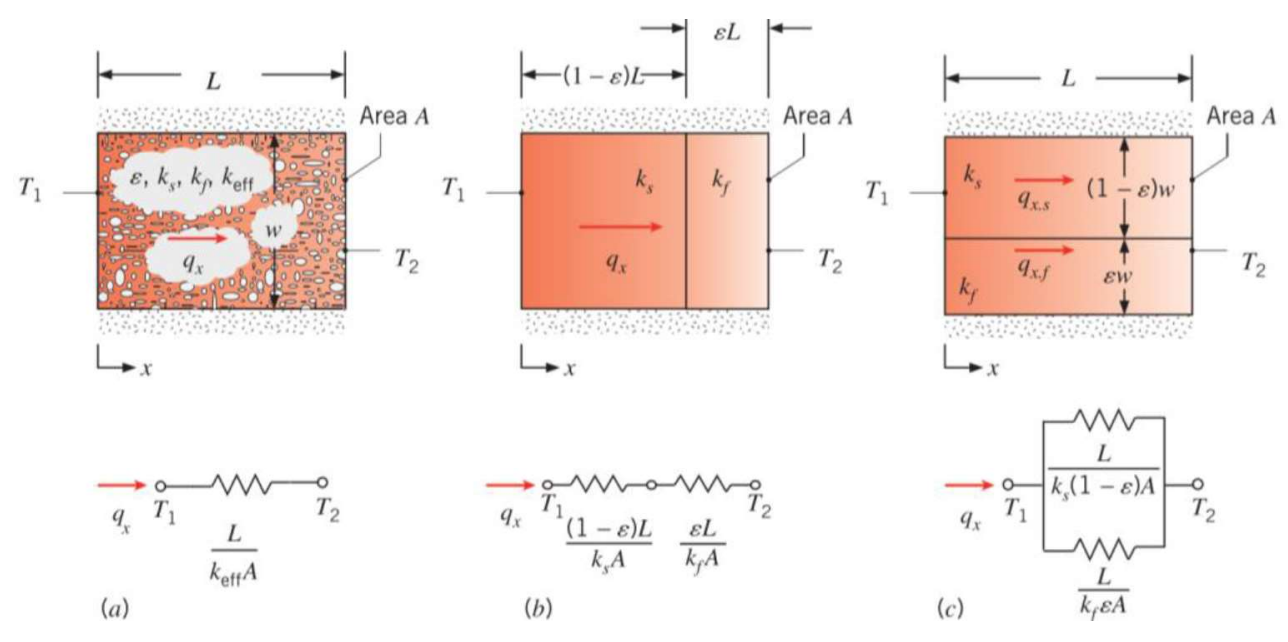

Figure 2.9: A porous medium. (a) The medium and its properties; (b) Series thermal resistance representation; (c) Parallel resistance representation (Bergman et al., 2011). $\varepsilon$ is the porosity of the medium.

Understanding the heat transfer and energy balance in porous media is important for thermal remediation technologies, such as thermal conduction heating, since they require excessive energy inputs to increase the in situ temperature to a desired value. In addition to the simple conduction and convection mechanisms introduced above, in practice, the actual temperature changes are affected by the changing thermophysical properties, e.g., heat capacities and conductivities, and by phase change processes, especially water evaporation and condensation in remediation applications (Stegemeier and Vinegar, 2001). Furthermore, temperature dependence of fluid properties (e.g., density and viscosity) and effects of heterogeneous geology also add to the complexity in understanding heat transfer through porous media for thermal remediation applications.

\subsection{Self-sustaining for Active Remediation (STAR)}

\subsubsection{Smouldering Combustion}

Smouldering is a slow, controllable and flameless form of combustion, which is sustained by several heterogeneous, exothermic reactions where oxygen directly attacks the surface 
of a solid or liquid fuel (Ohlemiller, 1985; Switzer et al., 2009). As for liquid fuels, a solid porous medium is critical for smouldering, self-sustained smouldering requires a high specific surface area and a permeable pathway to deliver the oxidant (air) (Akkutlu and Yortsos, 2003; Rein, 2009). The key difference between smouldering and flaming combustion is that flaming combustion is the oxidation of the gas phase around the fuel (Rein, 2009), whereas the fuel remains in the condensed phase when smouldering. One of the most common examples of smouldering combustion is the burning observed in charcoal barbeques (Figure 2.10).

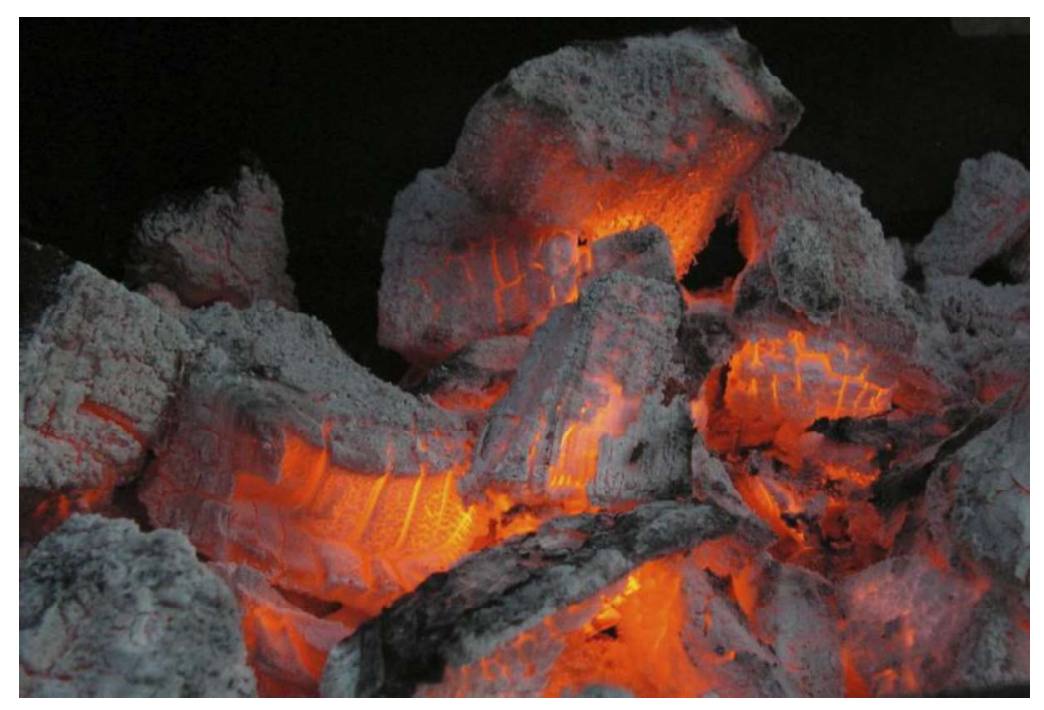

Figure 2.10: A charcoal barbeque is a common example of smouldering from Nielsen (2006).

Smouldering of complex fuels likely contains many chemical reactions, but the overall combustion can usually be simplified by considering the two most important chemical pathways: i.e., pyrolysis and oxidation (Rein, 2013):

Pyrolysis:

Fuel (Solid) + Heat $\rightarrow$ Pyrolysate $($ gas $)+$ Char $($ Solid $)+$ Ash (Solid) 
Char Oxidation:

Char (Solid) $+\mathrm{O}_{2} \rightarrow$ Heat $+\mathrm{CO}_{2}+\mathrm{H}_{2} \mathrm{O}+$ Ash (Solid) + Other Compounds

Pyrolysis is the thermochemical degradation of a fuel by heating without oxidation in an oxygen-limited environment, which results in gaseous (pyrolysate), and solid products (char) (Blasi, 1993). Typically, long chain molecules are broken into smaller molecules during pyrolysis, where the char is a carbon rich porous solid, and the pyrolysate is a complex mixture of molecules including volatile organic compounds (VOCs), light hydrocarbons, polyaromatic hydrocarbons, etc. Since pyrolysis is an endothermic reaction, the products are sensitive to the heating rate and peak temperature (Sinha et al., 2000), which are generally governed by the energy generation rate from the oxidation of char smouldering.

Oxidation is an exothermic degradation of the solid char produced from pyrolysis, or of the virgin fuel, that drives smouldering. If the fuel is completely oxidized, only water vapour and carbon dioxide will be produced (Rein, 2013). However, smouldering is an inherently incomplete form of combustion because of its characteristics (e.g., lower peak temperature) and complex natural conditions, and thus some typical incomplete combustion products are produced, e.g., carbon monoxide and unburnt light hydrocarbons (Rein, 2009, 2013).

\subsubsection{STAR as a NAPL Remediation Technology}

As discussed previously, conventional remediation methods used at NAPL contaminated site are challenging and typically involve physical excavation or chemical injection that are costly, labour intensive, and risky with the potential for secondary pollution. As an 
alternative, STAR, is a patented, smouldering-based technology developed to treat primarily heavy hydrocarbon NAPL contaminated sites.

As many heavy hydrocarbon NAPLs exhibit higher calorific values than other materials that are successfully burned in industries, these compounds may support robust smouldering combustion (Beever, 1986). As NAPL contaminated sites contain liquid fuel (NAPL) embedded in the soil (inert porous medium), this configuration readily supports smouldering combustion. This is because the porous medium increases the exposed surface area of NAPLs for oxidation, the soil permeability provides a pathway for the air to travel to the reaction zone, and the thermophysical properties of the sand store the energy released from smouldering and efficiently transfer it to unburnt NAPL to facilitate self-sustained smouldering (Switzer et al., 2009). Therefore, STAR takes advantage of the chemical energy embedded within the NAPLs, as well as the physical properties of the porous media to propagate a smouldering reaction through contaminated soil for remediation purposes. However, as showed in Equation 8, oxidation is limited by the supply of oxygen, and the energy is transferred from the oxidation zone to the unburnt fuel zone. Therefore, smouldering propagation is controlled by oxygen supply rate and heat transfer within the porous media (Ohlemiller, 1985; Torero and Fernandez-Pello, 1996).

Figure 2.11 shows a conceptual model of STAR. First, a localized region of contaminated soil is pre-heated near the base heating element until its ignition temperature (Figure 2.11a). Next, the forced air is injected into the pre-heated soil and ignites the contaminants; at the same time, the energy released from the smouldering is transferred to pre-heat and ignite unburnt fuels via conduction, convection, and radiation as the smouldering reaction propagates upwards (Figure 2.11b). The heating element is turned off once the smouldering 
reaction is deemed to be self-sustained, and the reaction continues to propagate through the contaminated soil, driven by a forced air flux (Figure 2.11c). Since the smouldering is sustained by the chemical energy released from the contaminants, the reaction selfterminates when all contaminants are destroyed, or if conditions occur ahead of the smouldering reaction that may cause extinction (e.g., large moisture contents) (Figure 2.11d).

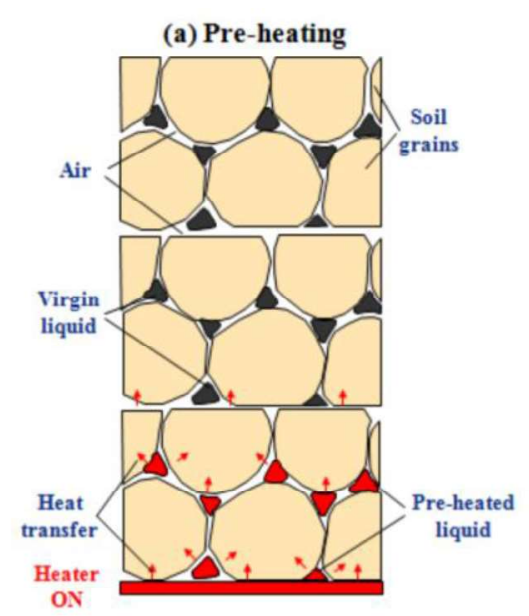

(c) Self-Sustained Smouldering

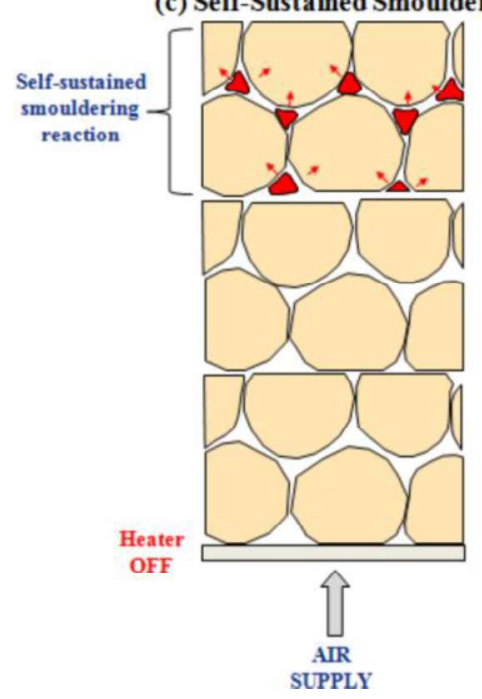

(b) Ignition
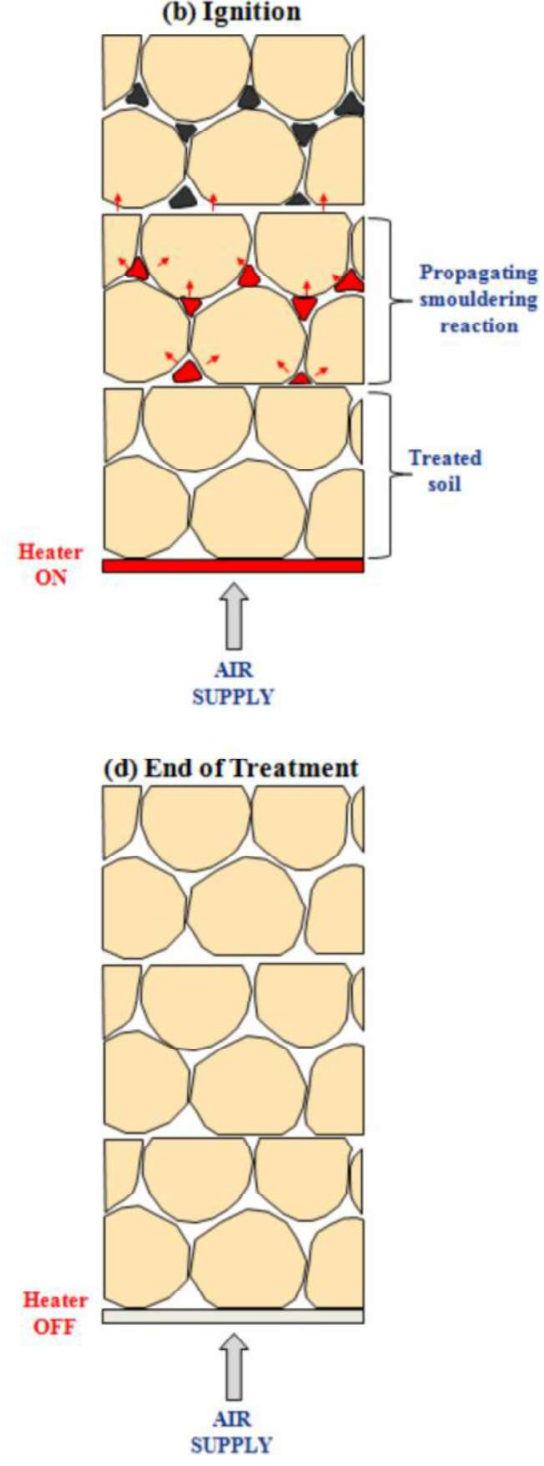

Figure 2.11: Conceptual model of STAR: (a) preheating, (b) ignition, (c) self-sustained smouldering and (d) the end of treatment (Solinger, 2016). 


\subsubsection{Parameters Affecting Smouldering Performance}

A series of proof-of-concept experiments were first conducted in small columns $(100 \mathrm{~mm}$ in diameter and $50 \mathrm{~mm}$ in height to $138 \mathrm{~mm}$ in diameter and $150 \mathrm{~mm}$ in height) to assess if self-sustained smouldering could be used to remediate contaminated soils. The results showed that self-sustained smouldering can be achieved across a broad range of different soil types and contaminants (Pironi et al., 2009; Switzer et al., 2009). Most of these laboratory experiments were set up based on the configuration showed in Figure 2.12. The smouldering propagation was monitored by thermocouples (TCs) positioned along the central axis of the column. The insulation near the column wall was used to minimize heat losses out of the column, so to best simulate conditions at a field site, which would have negligible heat losses.

Based on the initial experiments, later studies examined the sensitivity of STAR to several key parameters, which are briefly reviewed here.

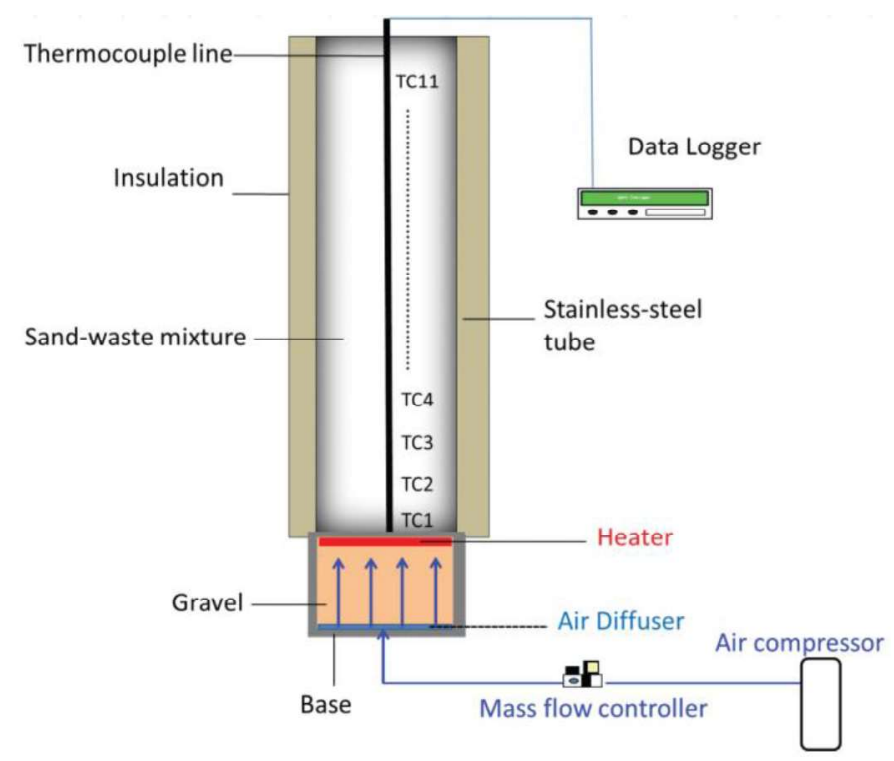

Figure 2.12: Schematic representation of the reaction system used in the smouldering of waste (Yermán et al., 2016). 


\section{(1) Moisture Content}

Moisture content is an important energy sink that affects the ignition and limits of selfsustained smouldering. If the reaction is not robust, i.e., it is close to extinction, peak temperatures decrease as the moisture content increases, eventually leading to extinction (Yermán et al., 2016). If the reaction is robust, small amounts of water can be easily evaporated by hot gases ahead of the smouldering zone. However, if the amount of water exceeds a limiting value, there is a potential that water ahead of the reaction may flow downwards into the reaction, e.g., in Figure 2.12, quenching the smouldering (Yermán et al., 2016). Rashwan et al. (2016) determined the upper limit of moisture content in biosolids that would support successful smouldering was $80 \%$ by mass.

\section{(2) Scale}

System scale is another critical parameter that can affect the heat retained in a smouldering system. Generally, small-scale columns are more susceptible to heat losses than larger columns because of their higher surface area to volume ratio. Switzer et al. (2014) explored the effects of changing heat losses with system scale in a series of experiments in reactors ranging from $0.003 \mathrm{~m}^{3}$ to $3 \mathrm{~m}^{3}$. The results showed that the smouldering reaction reached the entire contaminated volume in all reactors with similar velocities, peak temperatures, and remediation efficiencies between $97-99.5 \%$ were achieved in all experiments. Besides, it also revealed that minimum fuel concentration for self-sustained smouldering may be lower in larger scale systems than observed at bench scale systems. Scholes et al. (2015) completed the first pilot scale test of STAR, achieving a radius of influence of $3.7 \mathrm{~m}$ in the deep test $7.9 \mathrm{~m}$ below the ground surface, and destroying $860 \mathrm{~kg}$ of coal tar over 11 days. The contaminant removal was between $97.3 \%$ and $99.3 \%$ in this case. Although Kinsman 
et al. (2017) found the potential downward organic liquid migration in the tall treatment system $(90 \mathrm{~cm})$ at the air flux less than $3 \mathrm{~cm} / \mathrm{s}$, smouldering was demonstrated to overcome the downward migration and thus still destroyed all organic wastes in the system. These experiments showed that, though heat losses varied between the different reactors, the reported smouldering metrics of robust systems (i.e. far from extinction limits) were insensitive to scale and other experimental conditions.

\section{(3) Airflow Rate}

Smouldering is an oxygen-limited reaction. One early analytical model of the onedimensional, steady smouldering showed that: 1) The peak smouldering temperature depended on the initial oxygen mass flux and increased logarithmically with it and 2) The propagation velocity increased linearly with the oxygen mass flux (Dosanjh et al., 1987). In addition, Yermán et al. (2016) studied the effect of airflow rate on the smouldering of feces mixed with sand, where the smouldering reaction velocity increased linearly as the increase of airflow rate. However, though the peak temperatures also increased logarithmically at low airflow rates, they decreased at higher airflow rates, which suggested that some phenomena at high airflows were not embedded in the model from Dosanjh et al. 1987 (Figure 2.13). The observations reveal that high airflows increase reaction temperatures whereas, at some point, convective cooling occurs, which weakens the smouldering reaction and can eventually lead to extinction. However, it is noted that the airflows associated with cooling are above $20 \mathrm{~cm} / \mathrm{s}$; whereas the airflow rates typically applied in smouldering remediation are $0.5-8 \mathrm{~cm} / \mathrm{s}$ and in this range increased airflow only results in more robust conditions (Yermán et al., 2016). 


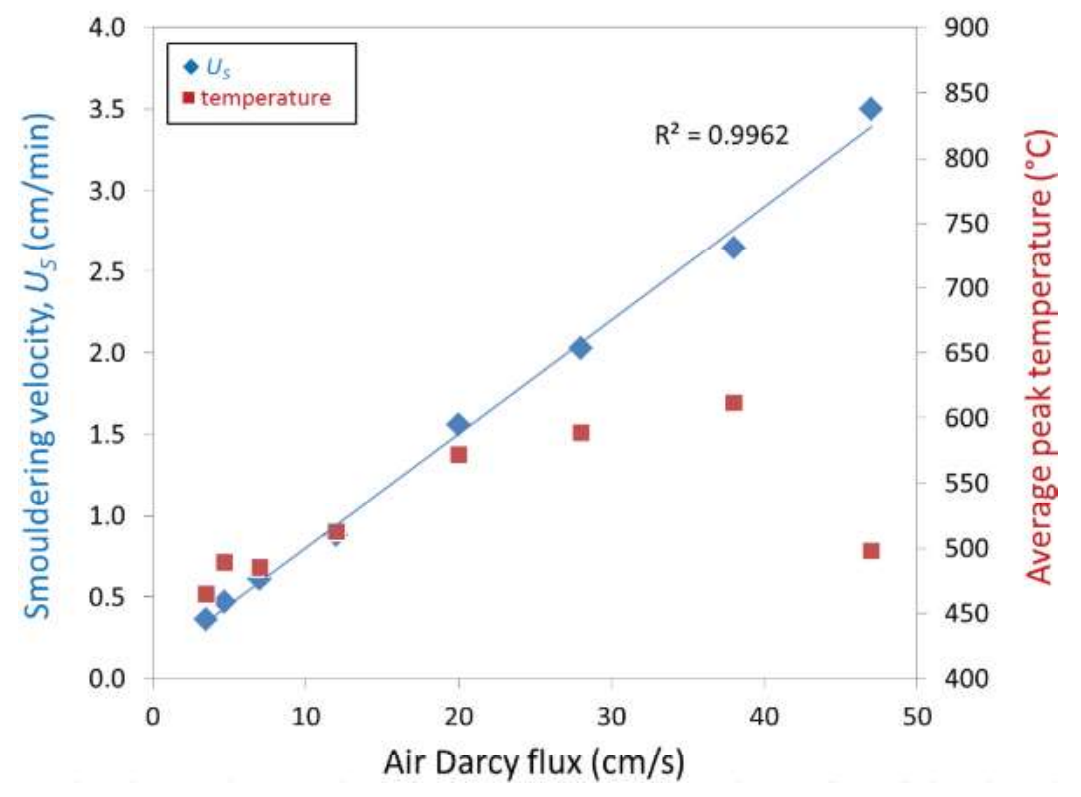

Figure 2.13: Peak temperature and smouldering velocity as a function of Darcy flux for smouldering of feces mixed with sand (Yermán et al., 2016).

In addition, since smouldering requires oxygen to sustain the exothermic oxidation reactions, there is a minimum oxygen mass flux required for self-sustained smouldering propagation. Pironi et al. (2011) observed self-sustained smouldering for both coal tar and crude oil was achievable down to a limiting air flux of $0.5 \mathrm{~cm} / \mathrm{s}$. MacPhee et al. (2012), Hasan et al. (2015) and Solinger et al. (2019) presented a phenomenological in situ smouldering model (ISSM) capable of simulating the evolution of smouldering reaction, which coupled a three-dimensional, multi-phase DNAPL flow model (Gerhard and Kueper, 2003a, 2003b, 2003c) with a combustion reaction expansion model (Richards, 1990, 1995) (see summary of ISSM in Appendix E). The model used the same air flux threshold (0.5 $\mathrm{cm} / \mathrm{s}$ ) to predict the extent of smouldering reaction propagation, and was validated in several one- and two-dimensional experiments. Physically, below this low air flux threshold, the local energy losses at the smouldering reaction exceed the energy generated 
and the reaction dies (Zanoni et al., 2019). It is expected that the lower airflow threshold may be even smaller at larger scales, but this has yet to be proven.

\section{(4) Sand Grain Size}

As grain size increases in a homogeneous porous medium system, it takes more energy to heat large sand grains and takes more time for the energy to penetrate and equilibrate in the grain. Besides, when a system consists larger grains, the surface area to volume ratio diminishes, resulting in the decrease of the amount of energy generation per volume in the smouldering system (Pironi et al., 2011; Yermán et al., 2016). Pironi et al. (2011) studied the effect of the size of sand particles on smouldering various NAPLs (i.e., coal tar and crude oil) in a $275 \mathrm{~mm}$ height quartz glass column by using different porous media. The results showed both the smouldering velocity and peak temperature decreased in $10 \mathrm{~mm}$ gravel column where the reaction was not self-sustained, compared to the robust smouldering in $6 \mathrm{~mm}$ gravel column.

At the other extreme, small particle diameters have led to problems in achieving selfsustained smouldering, presumably due to their associated low pneumatic permeability. Yermán et al. (2016) investigated this phenomenon by smouldering of feces embedded within various types of porous media, and showed unsuccessful smouldering occurred when the sand particle size was below $0.5 \mathrm{~mm}$. However, the result of Yermán et al. (2016) was not clear because the ignition protocol may have some influences whether the fine layers were ignited properly or not. Self-sustained smouldering should be assessed on the performance of the reaction away from the boundaries. So far, the fundamental reason behind non-self-sustained smouldering in fine, (presumably) homogeneous media has not been addressed in the literature. 


\section{(5) Heterogeneity}

Smouldering remediation is most successfully applied within a thick deposit of homogeneous granular soil; however, real geological systems have substantial heterogeneities. Similar to the challenges faced by other remediation technologies, heterogeneity also affects smouldering treatment. For example, in one in situ smouldering test, preferential smouldering through the coarse brick layer instead of the surrounding contaminated soil was observed (Scholes et al., 2015). Since sections of contaminated soil were left untreated due to subsurface heterogeneity, it is important to understand what degree and style of heterogeneity lead to the poor treatment by smouldering, and how engineers can address the challenge.

However, few studies have been carried out to investigate the effects of intrinsic permeability and heterogeneity on smouldering performance. Akkutlu and Yortsos (2005) developed an analytical model to study on the propagation of in situ combustion, an oil recovery process based on smouldering, in layered porous media where two layers of different permeabilities were separated by an interface impermeable to air (Figure 2.14). Based on this study, it was found that conductive heat transfer between two layers, termed "thermal coupling", greatly retarded the combustion reaction in the high permeability zone and slightly accelerated the reaction in the low permeability zone. This was explained as the released reaction energy in the high permeability zone was transferred to the oil-laden rock ahead of the reaction in the low permeability layer. 


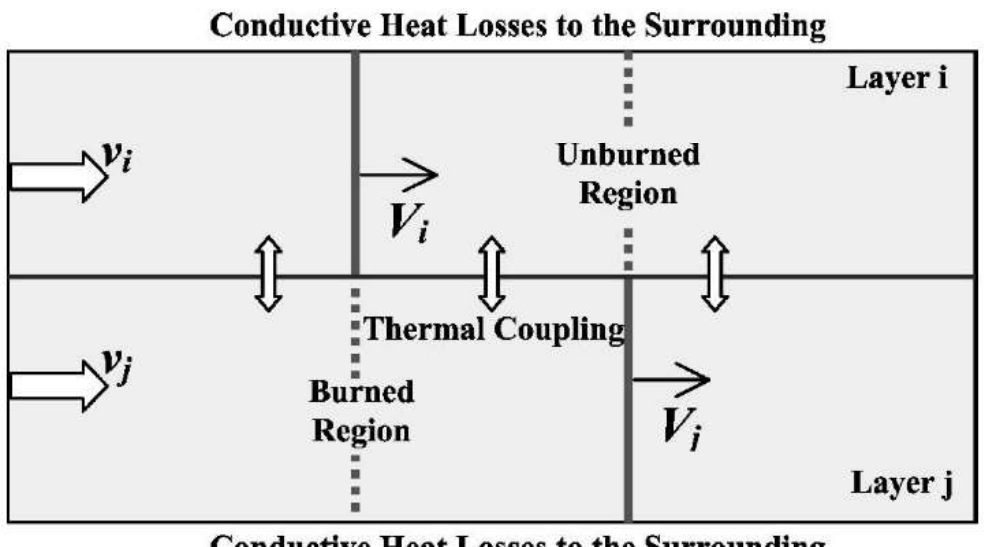

Conductive Heat Losses to the Surrounding

Figure 2.14: Schematic of the notation used for the propagation of combustion reaction in a two-layered porous medium. $v_{i}$ and $v_{j}$ are the air injection velocities in two layers, $V_{i}$ and $V_{j}$ are the dimensionless front velocities normalized with injection velocities in two layers (Akkutlu and Yortsos, 2005).

MacPhee et al. (2012), Hasan et al. (2015), and Solinger et al. (2019) employed a model, the ISSM, to simulate the evolution of a smouldering reaction in heterogeneous porous media. The model was demonstrated to reliably predict the propagation of the reaction under complex air injection flow fields in a homogeneous medium, including reaction termination in regions where air flux was too low to support self-sustained smouldering (Hasan et al., 2015). Figure 2.15 shows the model-predicted development of smouldering in the presence of two low permeability lenses $\left(1.9 \times 10^{-11} \mathrm{~m}^{2}\right.$ and $\left.1.9 \times 10^{-12} \mathrm{~m}^{2}\right)$, in which the smouldering reaction propagated into $1.9 \times 10^{-11} \mathrm{~m}^{2}$ lens at a slower rate compared to surrounding permeable region and was unable to enter $1.9 \times 10^{-12} \mathrm{~m}^{2}$ lens. This test was numerical only, to evaluate model behaviour, and was not confirmed with any experiments. Solinger et al. (2019) conducted modelling which suggested that increased permeability variance could degrade remediation performance because of increased airflow channelling. Although this model appears capable of simulating smouldering reaction propagation under heterogeneous situations, numerous system 
properties such as water content, temperature dependence of fluid properties, and heat transfer (such as thermal coupling) are not considered in the model, since it does not solve the energy equation (MacPhee et al., 2012). Moreover, all validation experiments were in homogeneous media and heterogeneous smouldering experiments have not, to the author's knowledge, been conducted.

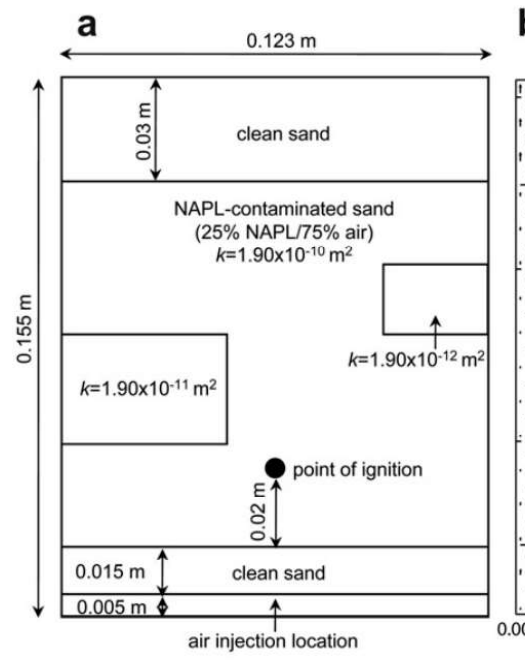

b c
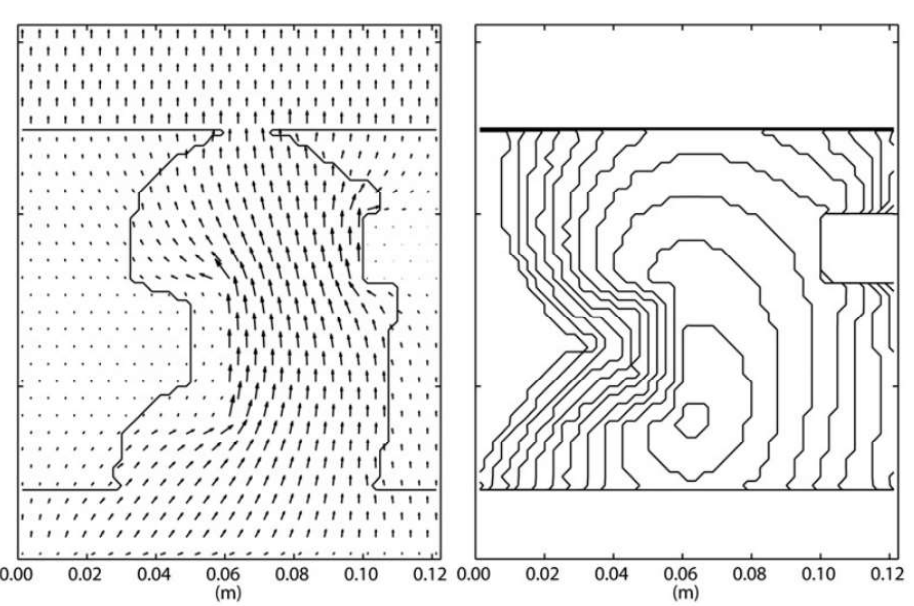

Figure 2.15: Simulation (low permeability regions): (a) model domain set-up, (b) distribution of air (vector size range: $0.00-0.430 \mathrm{~m} / \mathrm{s}$ ) and position of the smouldering reaction at $625 \mathrm{~s}(10.4 \mathrm{~min})$ following ignition, (c) contour plot depicting the position of the smouldering reaction at $125 \mathrm{~s}(2.1 \mathrm{~min})$ intervals from $t=0 \mathrm{~s}$ to $1500 \mathrm{~s}(25.0$ min) (MacPhee et al., 2012).

\subsection{Conclusion}

Due to the characteristic of NAPL and complexity of the subsurface environment, complete NAPL remediation from a contaminated site is costly and labour intensive. STAR is a promising and effective technology for NAPL remediation because of its features: minimal energy input, high degree of destruction, relatively fast treatment, ease of control, etc. The success of this technology has been demonstrated both at the laboratory and field scales. 
While there are several studies on the characteristic of fuels (e.g., various NAPLs, moisture content), relatively little is known about the effect of porous media heterogeneity on smouldering experiments. Akkutlu and Yortsos (2005) developed an analytical model considering the in situ combustion performance in layered porous media where only heat transfer between layers was considered. The ISSM is also able to predict the smouldering evolution in heterogeneous porous media through a phenomenological approach. However, there is currently a knowledge gap, where there are no experimental studies considering permeability heterogeneity in a smouldering system.

This work presents a series of experiments that explore how a smouldering reaction responds to permeability heterogeneity. It employed experiments packed with soils with a range of permeabilities layered in series, in parallel, and in a complex pattern (including both series and parallel layers) relative to the direction of airflow. The results provide unique insights in understanding the effect of permeability heterogeneity on smouldering propagation and have practical implications toward the design of full-scale smouldering remediation systems.

\subsection{References}

Akkutlu, I. Y., \& Yortsos, Y. C. (2003). The dynamics of in-situ combustion fronts in porous media. Combustion and Flame, 134 (3), 229-247.

Akkutlu, I. Y., \& Yortsos, Y. C. (2005). The Effect of Heterogeneity on In-Situ Combustion: Propagation of Combustion Fronts in Layered Porous Media. SPE Journal, 10(04), 394-404.

ASHRAE. (1997). 1997 Ashrae handbook - fundamentals. Atlanta, GA. 576 p.

Atlas R. M., \& Philp, J. C. (2005). Bioremediation: applied microbial solutions for realworld environmental cleanup. American Society for Microbiology (ASM) Press, Washington, 139-236. 
Beever, P. F. (1986). Initiation and propagation of smoldering reactions. (PhD.), University of Leeds, Leeds, England.

Bejan, A., \& Lage, J. L. (1991) Heat transfer from a surface covered with hair. Convective Heat and Mass Transfer in Porous Media. Kluwer Academic, Dordrecht, 823-845

Bergman, T. L., Lavine, A. S., Incropera, F. P., \& Dewitt, D. P. (2011). Fundamentals of heat and mass transfer. Hoboken: J. Wiley \& Sons. 1048 p.

Berkowitz, B., Cortis, A., Dentz, M., \& Scher, H. (2006). Modeling non-Fickian transport in geological formations as a continuous time random walk. Reviews of Geophysics, 44(2).

Blasi, C. Di. (1993). Modeling and simulation of combustion processes of charring and non-charring solid fuels. Progress in Energy and Combustion Science, 19(1), 71104.

Brecht, A. V., Hens, H., Lemaire, J.-L., Aerts, J. M., Degraeve, P., \& Berckmans, D. (2005). Quantification of the heat exchange of chicken eggs. Poultry Science, 84(3), 353-361.

Carman, P. C. (1937) "Fluid flow through granular beds." Transactions, Institution of Chemical Engineers, London, 15, 150-166.

Carman, P. C. (1956). Flow of gases through porous media. Butterworths, London. 182 p.

Coutelieris, F. A., \& Delgado, J. M. P. Q. (2012). Transport processes in Porous media. Advanced structured materials. New York: Springer. $236 \mathrm{p}$.

Darcy, H. P. G. (1856). In: Dalmont, V., Ed., Les Fontaines Publiques de la Ville de Dijon: Exposition et Application des Principes a Suivre et des Formulesa Employer dans les Questions de Distribution d'Eau. Paris. 647 p.

Domenico, P. A., \& Schwartz, F, W. (1998). Physical and chemical hydrogeology. John Wiley \& Sons. Inc. 528 p.

Dosanjh, S. S., Pagni, P. J., \& Fernandez-Pello, A. C. (1987). Forced cocurrent smoldering combustion. Combustion and Flame, 68(2), 131-142.

ESTCP. (2001). Use of cometabolic air sparging to remediate chloroethenecontaminated groundwater aquifers: Environmental security technology certification program.

Fiori, A., Dagan, G., Jankovic, I., \& Zarlenga, A. (2013). The plume spreading in the MADE transport experiment: Could it be predicted by stochastic models? Water Resources Research, 49(5), 2497-2507. 
Gerhard, J. I., \& Kueper, B. H. (2003a). Capillary pressure characteristics necessary for simulating DNAPL infiltration, redistribution, and immobilization in saturated porous media. Water Resources Research, 39(8).

Gerhard, J. I., \& Kueper, B. H. (2003b). Relative permeability characteristics necessary for simulating DNAPL infiltration, redistribution, and immobilization in saturated porous media. Water Resources Research, 39(8).

Gerhard, J. I., \& Kueper, B. H. (2003c). Influence of constitutive model parameters on the predicted migration of DNAPL in heterogeneous porous media. Water Resources Research, 39(10).

Happel, J., \& Brenner, H. (1986). Low Reynolds number hydrodynamics with special applications to particulate media. Nijhoff Publishers. $553 \mathrm{p}$.

Hasan, T., Gerhard, J. I., Hadden, R., \& Rein, G. (2015). Self-sustaining smouldering combustion of coal tar for the remediation of contaminated sand: Twodimensional experiments and computational simulations. Fuel, 150, 288-297.

Hird, J. (1993). Environmental Policy and Equity: The Case of Superfund. Journal of Policy Analysis and Management, 12(2), 323-343.

Hunt, A. G., Skinner, T. E., Ewing, R. P., \& Ghanbarian-Alavijeh, B. (2011). Dispersion of solutes in porous media. The European Physical Journal B, 80(4), 411-432.

Janković, I., Fiori, A., \& Dagan, G. (2006). Modeling flow and transport in highly heterogeneous three-dimensional aquifers: Ergodicity, Gaussianity, and anomalous behavior. Conceptual issues and numerical simulations. Water Resources Research, 42(6).

Kaviany, M. (1991). Principles of heat transfer in porous media. New York: Springer. $626 \mathrm{p}$.

Kinsman, L., Torero, J., \& Gerhard, J. (2017). Organic liquid mobility induced by smoldering remediation. Journal of Hazardous Materials, 325, 101-112.

Kozeny, J. (1927) Ueber kapillare Leitung des Wassers im Boden. Sitzungsber Akad. Wiss., Wien, 136(2a), 271-306.

Kueper, B. H., Stroo, H.F., Vogel, C. M., \& Ward, C. H. (2014). Chlorinated Solvent Source Zone Remediation. New York: Springer. 713 p.

Levy, M., \& Berkowitz, B. (2003). Measurement and analysis of non-Fickian dispersion in heterogeneous porous media. Journal of Contaminant Hydrology, 64(3-4), 203226.

MacPhee, S. L., Gerhard, J. I., \& Rein, G. (2012). A novel method for simulating smoldering propagation and its application to STAR (Self-sustaining Treatment for Active Remediation). Environmental modelling \& software, 31, 84-98 
Maitra, S. (2019). Permeable reactive barrier: a technology for groundwater remediation a mini review. Research Journal of Life Sciences, Bioinformatics, Pharamaceutical and Chemical Sciences, 5(1), 203-217.

Mercer, J. W., \& Cohen, R. M. (1990). A review of immiscible fluids in the subsurface: Properties, models, characterization and remediation. Journal of Contaminant Hydrology, 6(2), 107-163.

Naftz, D. L., Morrison, S. J., Davis, J. A., \& Fuller, C. C. (2002). Handbook of Groundwater Remediation using Permeable Reactive Barriers. USA: Elsevier Science. 544 p.

Nield, D. A., \& Bejan, A. (2013). Convection in porous media, New York: Springer. $778 \mathrm{p}$.

Obiri-Nyarko, F., Grajales-Mesa, S. J., \& Malina, G. (2014). An overview of permeable reactive barriers for in situ sustainable groundwater remediation. Chemosphere, $111,243-259$.

Ohlemiller, T. J. (1985). Modeling of smoldering combustion propagation. Progress in energy and combustion science, 11(4), 277-310.

Page, J. W., Soga, K., \& Illangasekare, T. (2007). The significance of heterogeneity on mass flux from DNAPL source zones: An experimental investigation. Journal of Contaminant Hydrology, 94(3-4), 215-234.

Pankow, J. F., \& Cherry, J. A. (1996). Dense chlorinated solvents and other DNAPLs in groundwater: history, behavior, and remediation. Portland, OR: Waterloo Press. $522 \mathrm{p}$.

Pironi, P., Switzer, C., Rein, G., Fuentes, A., Gerhard, J. I., \& Torero, J. L. (2009). Smallscale forward smouldering experiments for remediation of coal tar in inert media. Proceedings of the Combustion Institute, 32(2), 1957-1964.

Pironi, P., Switzer, C., Gerhard, J. I., Rein, G., \& Torero, J. L. (2011). Self-sustaining smoldering combustion for NAPL remediation: Laboratory evaluation of process sensitivity to key parameters. Environmental Science and Technology, 45(7), 2980-2986.

Pryor, W. A. (1973). Permeability-Porosity Patterns and Variations in Some Holocene Sand Bodies. AAPG Bulletin, 57(1), 162-189.

Rashwan, T. L., Gerhard, J. I., \& Grant, G. P. (2016). Application of self-sustaining smoldering combustion for the destruction of wastewater biosolids. Waste Management, 50, 201-212.

Richards, G. D. (1990). "An elliptical growth model of forest fire fronts and its numerical solution." International Journal for Numerical Methods in Engineering, 30(6), 1163-1179. 
Richards, G.D. (1995). "A General Mathematical Framework for Modeling TwoDimensional Wildland Fire Spread." International Journal of Wildland Fire, 5(2), 63-72.

Reddy, K. R., \& Adams, J. A. (2001). Effects of soil heterogeneity on airflow patterns and hydrocarbon removal during in situ air sparging. Journal of geotechnical and geoenvironmental engineering, 127(3), 234-247.

Rein, G. (2009). Smoldering combustion phenomena in science and technology. International Review of Chemical Engineering, 1, 3-18.

Rein, G. (2013). Smoldering fires and natural fuels. Fire Phenomena and the Earth System: An Interdisciplinary Guide to Fire Science, 15-33.

Rosenbaum, W. A. (2019). Environmental politics and policy. Washington, D.C.: CQ Press, a Division of Sage. 352 p.

Scheidegger, A.E. (1974). The Physics of Flow through Porous Media. University of Toronto Press, Toronto. 330 p.

Scherer, M. M., Richter, S., Valentine, R. L., \& Alvarez, P. J. J. (2000). Chemistry and Microbiology of Permeable Reactive Barriers for In Situ Groundwater Clean up. Critical Reviews in Microbiology, 26(4), 221-264.

Scholes, G.C., Gerhard, J.I., Grant, G.P., Major, D.W., Vidumsky, J.E., Switzer, C., \& Torero, J.L. (2015). Smoldering remediation of coal-tar-contaminated soil: pilot field tests of STAR. Environmental Science and Technology. 49, 14334-14342.

Schwartz, F.W., \& Zhang, H. (2003). Fundamentals of Groundwater. John Wiley \& Sons, New York. 592 p.

Seol, Y., Zhang, H., \& Schwartz, F. W. (2003). A Review of In Situ Chemical Oxidation and Heterogeneity. Environmental and Engineering Geoscience, 9(1), 37-49.

Sinha, S., Jhalani, A., Ravi, M., \& Ray, A. (2000). Modelling of pyrolysis in wood: A review. SESI Journal, 10(1), 41-62.

Solinger, R.L. (2016). STARx Technology for waste oil sludge treatment investigated with numerical modelling. (M.E.Sc.), The University of Western Ontario, London, ON.

Solinger, R., Grant, G.P., Scholes, G.C., Murray, C., \& Gerhard, J.I. (2019). STARx hottpad for smouldering treatment of waste oil sludge: proof of concept and sensitivity to key design parameters. Accepted for publication in Waste Management \& Research.

Stegemeier, G. L., \& Vinegar, H. J. (2001). Thermal conduction heating for in-situ thermal desorption of soils. Hazardous and radioactive waste treatment technologies handbook. CRC Press Boca Raton, Florida. 1-37 
Switzer, C., Pironi, P., Gerhard, J., Rein, G., \& Torero, J. (2009). Self-sustaining smoldering combustion: a novel remediation process for non-aqueous-phase liquids in porous media. Environmental Science \& Technology, 43(15), 5871-5877.

Switzer, C., Pironi, P., Gerhard, J. I., Rein, G., \& Torero, J. I. (2014). Volumetric scaleup of smoldering remediation of contaminated materials. Journal of Hazardous Materials, 268(15), 51-60.

Thiruvenkatachari, R., Vigneswaran, S., \& Naidu, R. (2008). Permeable reactive barrier for groundwater remediation. Journal of Industrial and Engineering Chemistry, $14(2), 145-156$.

Torero, J., \& Fernandez-Pello, A. (1996). Forward smolder of polyurethane foam in a forced air flow. Combustion and Flame, 106(1-2), 89-109.

U.S. EPA (U.S. Environmental Protection Agency). (1995). Ground Water Issue: Light nonaqueous phase liquids. EPA-540-S-95-500. Office of Research and Development. $28 \mathrm{p}$.

U.S. EPA (U.S. Environmental Protection Agency). (2000). Field demonstration of permeable reactive barriers to remove dissolved uranium from groundwater, fry canton, Utah. EPA-402-C-00-001. Office of Air and Radiation Emergency and Remedial Response. 98 p.

U.S. EPA (U.S. Environmental Protection Agency). (2002). Groundwater remedies selected at superfund sites. EPA-542-R-01-022. Office of Solid Waste and Emergency Response. 38 p.

U.S. EPA (U.S. Environmental Protection Agency). (2017). How to evaluate alternative cleanup technologies for underground storage tank sites. A guide for corrective action plan reviewers. EPA-510-B-17-003. Office of Land and Emergency Management. $47 \mathrm{p}$.

U.S. EPA (U.S. Environmental Protection Agency). (2018). Examples of Groundwater Remediation at NPL Sites. EPA-542-R-18-002. Office of Superfund Remediation and Technology Innovation (OSRTI). $114 \mathrm{p}$.

Whitaker, S. (1986). Flow in Porous Media I: A Theoretical Derivation of Darcy's Law. Transport in Porous Media, 1(1), 3-25.

Xu, P., \& Yu, B. (2008). Developing a new form of permeability and Kozeny-Carman constant for homogeneous porous media by means of fractal geometry. Advances in Water Resources, 31(1), 74-81.

Yermán, L., Hadden, R. M., Carrascal, J., Fabris, I., Cormier, D., Torero, J. L., Cheng, Y. (2015). Smoldering combustion as a treatment technology for faeces: Exploring the parameter space, Fuel, 147(1), 108-116.

Yermán, L., Wall, L., Torero, J., Gerhard, J. I., \& Cheng, Y. -L. (2016). Smoldering combustion as a treatment technology for feces: sensitivity to key parameters. Combustion Science and Technology, 188(6), 968-981. 
Zanoni, M. A., Torero, J. L., \& Gerhard, J. I. (2017). Determination of the interfacial heat transfer coefficient between forced air and sand at Reynold's numbers relevant to smouldering combustion. International Journal of Heat and Mass Transfer, 114, 90-104.

Zanoni, M. A., Torero, J. L., \& Gerhard, J. I. (2019). Delineating and explaining the limits of self-sustained smouldering combustion. Combustion and Flame, 201, 78-92. 


\section{Chapter 3}

\section{The Influence of Porous Media Heterogeneity on Smouldering Remediation}

\subsection{Introduction}

Non-aqueous phase liquid (NAPL) contamination is one of the most significant environmental challenges in the field of remediation (U.S. EPA, 2003). This contamination resulted from poor environmental regulations and the lack of appropriate disposal of industrial chemical compounds over the last century (Kueper et al., 2014). At present, there are an estimated 100, 000 contaminated sites in the United States that need costly remediation (NRC, 2013; Kueper et al., 2014), posing a high toxicity potential to residents as well as a heavy financial burden on stakeholders. With the improvement of environmental legislation since the 1970s, and a focus on restoring the environment and redeveloping brownfields, there was a surge of projects addressing historical contamination. Correspondingly, numerous NAPL remediation technologies have emerged over recent decades such as bioremediation, chemical oxidation and thermal desorption (U.S. EPA, 2002; Rosenbaum, 2019). Despite these developments, few sites have been restored to conditions that meet regulatory standards.

Self-sustaining Treatment for Active Remediation (STAR) is a remediation technology based on smouldering combustion. Smouldering is a flameless form of combustion that depends on heterogeneous exothermic reactions, i.e., gaseous oxygen interacts with the burning surface of condensed solid or liquid fuels to release energy (Ohlemiller, 1985). A common example of smouldering combustion is the glowing charcoal in a barbeque (Switzer et al., 2009; Pironi et al., 2011; Yermán et al., 2016). After a short-duration 
ignition event, smouldering can be a self-sustained process: excess energy generated from the reaction ignites the smouldering in adjacent areas. By this process, a smouldering reaction can propagate indefinitely along a path as long as fuel exists and oxygen can reach the reaction zone (Switzer et al., 2009).

STAR has been shown to effectively treat a range of NAPLs including crude oil, coal tar, chlorinated solvents, mixed hydrocarbons and oil drilling muds (Pironi et al., 2009, 2011; Switzer et al., 2009, 2014; Scholes et al., 2015; Salman et al., 2015; Grant et al., 2016). Smouldering requires the fuel to be distributed in a porous medium, which is essential for the oxidant (oxygen in air) to flow from the boundary (sample edge) to the reaction. The porous medium provides other benefits as well such as a high surface area for the reaction and high heat capacity to support energy storage and recycling near the reaction (Drysdale, 2011). In the case of smouldering NAPLs, the porous medium is the contaminated soil while the contaminant is the fuel.

Smouldering is typically an oxygen-limited reaction (Torero and Fernandez-Pello, 1996); therefore, it is not surprising that the rate of propagation of the reaction depends on the air mass flux. In practical terms, this means that there is a minimum airflow rate required for smouldering to be self-sustained. Laboratory and modelling studies (Pironi et al., 2011; MacPhee et al., 2012; Hasan et al., 2015; Solinger et al., 2019) have observed the minimum to be approximately $0.5 \mathrm{~cm} / \mathrm{s}$ (Darcy flux) for the smouldering of coal tar and crude oil. Below this threshold, the energy losses exceed the energy generated and stored such that the reaction dies (Zanoni et al., 2019). As airflow is increased above this threshold, a linear increase in the smouldering propagation rate is observed (Dosanjh et al., 1987; Yermán et al., 2016; Zanoni et al., 2019) in the range of flow rates achievable with typical air injection 
equipment. Note that the minimum air flux is likely a function of scale, and while $0.5 \mathrm{~cm} / \mathrm{s}$ applies to laboratory experiments, it is expected that lower air fluxes are possible in field scale applications where heat losses are reduced and thus smouldering is more energy efficient (Gerhard et al., 2020).

STAR has been applied as an in situ remediation method at numerous sites, including complete remediation of a large coal tar contaminated site in 2019. In these scenarios, the self-sustained reaction primarily travels along pathways determined by the dominant airflow vectors, including horizontally (parallel to bedding) as well as upwards (across bedding) (Scholes et al., 2015; Grant et al., 2016). Propagation of the smouldering reaction depends on forward convective heat transfer by the air; thus, the permeability of the porous medium is a critical variable. Indeed, most in situ remediation technologies that rely on fluid injection are sensitive to in situ permeability, including steam injection, bioremediation, and chemical oxidation (Reddy and Adams, 2001; Gill et al., 2014; Seol et al., 2003). STAR has been successfully applied within relatively homogeneous units, but strong permeability differences in the subsurface may be challenging. For example, Scholes et al. (2015) found that in a pilot field test of STAR, smouldering preferentially propagated through a high permeability layer of bricks in the subsurface.

Few studies have examined the effect of intrinsic permeability or soil heterogeneity on smouldering. Akkutlu and Yortsos (2005) developed an analytical solution for in situ combustion, an oil recovery process based on smouldering. That work examined the propagation of two reactions in adjacent parallel layers of contrasting permeability with airflow parallel to the layering. It revealed that conductive heat transfer between the layers, termed "thermal coupling", caused slowing of the reaction in the more permeable layer as 
energy was lost to the oil-laden rock ahead of the reaction in the less permeable layer. Solinger et al. (2019) applied a numerical model to predict the ex situ smouldering treatment of organic liquid wastes mixed with sands considering the heterogeneity of permeability and oil saturation. Simulations suggested that high permeability variance may degrade remedial performance because of enhanced channelling of airflow and thus smouldering, which has not been validated to experiments. Although these studies suggest soil heterogeneity has important influences on smouldering, they relied on analytical and numerical modelling. A laboratory investigation of smouldering under heterogeneous conditions has not been conducted.

This research aims to experimentally examine the influence of intrinsic permeability and heterogeneity on smouldering remediation. A series of laboratory smouldering experiments were conducted to examine the impact of contrasting porous medium layers arranged in series and in parallel relative to the direction of the reaction's propagation. The length and the permeability of layers within the treatment zone were varied to represent a range of heterogeneous scenarios. Extensive instrumentation allowed for tracking the smouldering reaction as well as air pressure gradients and fluxes in time and space across all layers. Simulation of a subset of the cases with a published in situ smouldering remediation numerical model (ISSM) assisted interpreting the results, which is the first study to validate this model to experimental results in certain cases. This work provides novel insights into the influence of intrinsic permeability and heterogeneity on the ability of smouldering to be a self-sustained remediation process. 


\subsection{Materials and Methodology}

The investigation was divided into four parts. First, the smouldering performance of the Base Case, a homogeneous experiment in coarse sand, was established. Second, fine layers of differing permeability were added to a coarse layer in series to study smouldering behaviour upon transitioning from a more-permeable to a less-permeable layer. Third, coarse and fine layers of varying permeability contrasts were arranged in parallel relative to the direction of airflow and thus to the direction of smouldering propagation. Fourth, coarse and fine layers were placed in a more complex pattern (checkerboard) to consider the effect of combining layers in series and parallel on smouldering, where coarse layers were disconnected. The concept of flow in series and parallel are illustrated in Figure 3.1, which used the analogy of resistors arranged in an electrical circuit, as is common for hydrogeological systems (Gorokhovski, 2012).

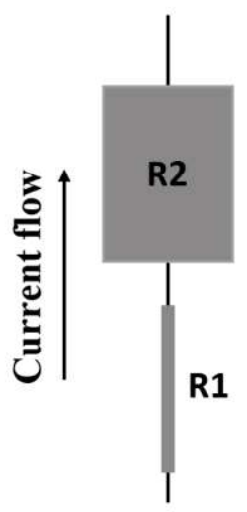

(a)

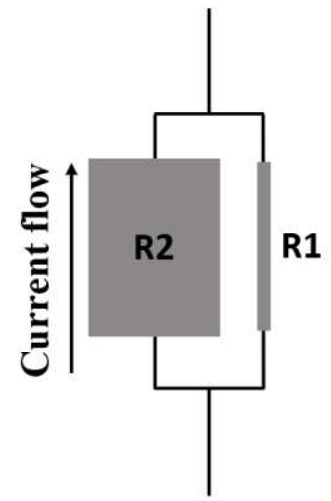

(b)

Figure 3.1: Analogies of the experiments conducted using circuits with (a) resistors in series, and (b) resistors in parallel. $\mathrm{R} 1$ is low resistance (representing the high permeability lens, coarse sand) and $\mathrm{R} 2$ is high resistance (representing the low permeability lens, fine sand). The current flow is analogous to the airflow and the voltage drop is analogous to the pressure drop. 


\subsubsection{Materials}

Three silica sands from Bell \& MacKenzie Co. Ltd. were used: \#12 Coarse Silica Sand (particle density is $2.65 \mathrm{~g} / \mathrm{m}^{3}$, mean grain diameter is $0.88 \mathrm{~mm}$ ), \#505 Fine Silica Sand (particle density is $2.65 \mathrm{~g} / \mathrm{m}^{3}$, mean grain diameter is $0.19 \mathrm{~mm}$ ), \#106 Powder Silica Sand (particle density is $2.65 \mathrm{~g} / \mathrm{m}^{3}$, mean grain diameter is $0.043 \mathrm{~mm}$ ). The sieve and hydrometer analyses for these are shown in Appendix A. To maximize homogeneity within each lens packed in the experiments, both coarse and fine sands were sieved to retain only a single sieve grain size: $1.18-2.00 \mathrm{~mm}$ in diameter for the coarse and $0.125-0.250 \mathrm{~mm}$ in diameter for the fine sand.

Mixing various weight fractions of the sieved coarse and fine sands with the silica powder allowed the production of a wide range of controlled intrinsic permeability values. Mixing (KitchenAid, Artisan Stand Mixer) was continued until the mixture was visually homogeneous (approximately five minutes), and permeability tests on many subsamples were conducted to ensure the batches were homogeneous (see Appendix A). Table A-1 in Appendix A summarizes the measured intrinsic permeability and porosity of 33 different mixing ratios of the three base sands. Intrinsic permeability was measured in a pneumatic permeability cell following ASTM D6539-00. In the smouldering experiments, the sieved coarse and sieved fine sands themselves were employed, as well as five chosen from these 33 mixtures (details below).

Granular Activated Carbon (GAC, Technical-grade chemicals, McMaster-CARR, Part number: $3190 \mathrm{~K} 523$ ) was used as the organic contaminant (i.e., fuel) in this study. GAC is often used in the remediation industry to sorb and concentrate dissolved organic contaminants, such as chlorinated solvents and Per- and polyfluoroalkyl substances 
(PFAS), after which it is contaminated itself and needs disposal or treatment (Carter and Farrell, 2010; Liu et al., 2019). Recent work has demonstrated that GAC mixed with sand produces a robust smouldering reaction, with self-sustained temperatures that exceed those expected to destroy a wide range of those contaminants including PFAS (Major, 2019). GAC was chosen for this study because it reproduces the smouldering behaviour observed for a wide range of common organic contaminants, such as coal tar, bitumen, and crude oil. However, GAC is much easier to use, is non-toxic to handle in the laboratory, and it requires much lower concentrations to achieve similar smouldering temperatures. In addition, the difference in pack permeability due to GAC presence (before smouldering) and absence (after smouldering) is minor (data below).

The GAC was characterized by conducting a proximate analysis: moisture content of $3.2 \%$ (ASTM-D2867-17), volatile matter content of 3.2\% (ASTM-D5832-98), ash content of 2.2\% (ASTM-D2866-11), and fixed carbon content of 91.4\% (calculated by difference). The higher heating value (HHV) for the GAC was $30.9 \mathrm{MJ} / \mathrm{kg}$, and the lower heating value (LHV) was $30.8 \mathrm{MJ} / \mathrm{kg}$; the small difference between them was due to the minimal moisture content of the GAC.

The seven sand mixtures used in the smouldering experiments (out of the 33 possibilities) are presented in Table 3.1, where the percentages shown are weight fractions. The permeability of each mixture is reported in two ways. "Cell" values are those measured in the pneumatic permeability cell. "In situ" values are those measured within the smouldering column when it was cold (methodology provided in Section 3.2.2 below) and include values for sands both with and without GAC at the employed concentration $(30 \mathrm{~g}$ $\mathrm{GAC} / \mathrm{kg}$ sand). The table reveals that the permeability cell provides values that are similar 
(within a half-order of magnitude) to the in situ values. Moreover, the table confirms that GAC has a minor influence on the intrinsic permeability.

Table 3.1: Measured Permeability of Seven Sands Used

\begin{tabular}{|c|c|c|c|c|c|c|}
\hline \multirow{3}{*}{ Sand } & \multirow{3}{*}{$\begin{array}{c}\text { Sieved Coarse } \\
(1.18-2.00 \mathrm{~mm})\end{array}$} & \multirow{3}{*}{$\begin{array}{c}\text { Sieved Fine } \\
(0.125-0.250 \mathrm{~mm})\end{array}$} & \multirow{3}{*}{$\begin{array}{c}\text { Powder } \\
(\bar{d}=0.043 \mathrm{~mm})\end{array}$} & \multicolumn{3}{|c|}{ Measured sand $\mathrm{k}\left(\mathrm{m}^{2}\right)$} \\
\hline & & & & \multirow{2}{*}{$\begin{array}{c}\text { Cell } \\
\text { No GAC }\end{array}$} & \multicolumn{2}{|c|}{ In situ } \\
\hline & & & & & No GAC & With GAC \\
\hline 1 & $100.0 \%$ & $0.0 \%$ & $0.0 \%$ & $1.3 \times 10^{-9}$ & $1.8 \times 10^{-9}$ & $6.7 \times 10^{-10}$ \\
\hline 2 & $80.0 \%$ & $20.0 \%$ & $0.0 \%$ & $4.5 \times 10^{-10}$ & - & - \\
\hline 3 & $60.0 \%$ & $40.0 \%$ & $0.0 \%$ & $1.2 \times 10^{-10}$ & - & - \\
\hline 4 & $0.0 \%$ & $100.0 \%$ & $0.0 \%$ & $1.9 \times 10^{-11}$ & $3.2 \times 10^{-11}$ & $2.3 \times 10^{-11}$ \\
\hline 5 & $50.0 \%$ & $45.0 \%$ & $5.0 \%$ & $9.7 \times 10^{-12}$ & $1.7 \times 10^{-11}$ & $1.1 \times 10^{-11}$ \\
\hline 6 & $45.0 \%$ & $45.0 \%$ & $10.0 \%$ & $2.0 \times 10^{-12}$ & $5.6 \times 10^{-12}$ & $3.9 \times 10^{-12}$ \\
\hline 7 & $42.5 \%$ & $42.5 \%$ & $15.0 \%$ & $1.1 \times 10^{-12}$ & $1.8 \times 10^{-12}$ & $1.3 \times 10^{-12}$ \\
\hline
\end{tabular}

\subsubsection{Experimental Set-up and Procedure}

Table 3.2 summaries the nine smouldering experiments performed in this study: One "single layer" test (Base Case), four "layers arranged in series" tests (Series 1 to 4), three "layers arranged in parallel" tests (Parallel 1 to 3), and one "layers arranged in a checkerboard" test (Complex: Disconnected Coarse), whose treatment zones were packed as shown in Figure 3.2. All experiments used $30 \mathrm{~g} \mathrm{GAC} / \mathrm{kg}$ sand. The GAC was mixed into the chosen sand mixture until homogeneous. Here sand well-mixed with GAC in both coarse and fine layers with the same concentration was used to mimic some field situations, this includes NAPL entering low permeability layers in situ (NAPL pressure > displacement pressure of fine layers) and well-mixed contaminated low permeability soils in ex situ applications. In addition, all experiments used the same injected air flux: $2.5 \mathrm{~cm} / \mathrm{s}$, the volume of air per unit cross-sectional area of column per time (i.e., Darcy flux). Air injection was fixed by a mass flow controller (FMA 5400/5500 Series, Omega Ltd.) connected to laboratory compressed air. 
Table 3.2: Summary of Smouldering Experiments

\begin{tabular}{|c|c|c|c|c|c|c|c|c|}
\hline \multirow{2}{*}{ Tests } & \multirow{2}{*}{$\begin{array}{c}\text { Coarse } \\
\text { layer }\end{array}$} & \multirow{2}{*}{$\begin{array}{c}\mathrm{H} \\
(\mathrm{cm})\end{array}$} & \multirow{2}{*}{$\begin{array}{l}\text { Fine } \\
\text { layer }\end{array}$} & \multirow{2}{*}{$\begin{array}{c}\text { h } \\
(\mathrm{cm})\end{array}$} & \multirow{2}{*}{$\begin{array}{c}k_{\text {ratio }}{ }^{c} \\
\left(k_{\text {Coarse }} / k_{\text {Fine }}\right)\end{array}$} & \multicolumn{2}{|c|}{ Treatment zone $k\left(\mathrm{~m}^{2}\right)$} & \multirow{2}{*}{ Smouldering } \\
\hline & & & & & & Measured $^{\mathrm{d}}$ & Calculated $^{\mathrm{e}}$ & \\
\hline Base Case & $\# 1^{\mathrm{a}}$ & 36.0 & None & 0.0 & NA & $1.8 \times 10^{-9}$ & $1.3 \times 10^{-9}$ & $\mathrm{SS}^{\mathrm{f}}$ \\
\hline Series 1 & None & 0.0 & $\# 5^{\mathrm{a}}$ & 63.0 & NA & $1.7 \times 10^{-11}$ & $9.7 \times 10^{-12}$ & SS \\
\hline Series 2 & $\# 1$ & 21.0 & $\# 4^{\mathrm{a}}$ & 21.0 & 68 & $5.7 \times 10^{-11}$ & $3.8 \times 10^{-11}$ & $\begin{array}{c}\text { SS in coarse layer } \\
\text { SS in fine layer }\end{array}$ \\
\hline Series 3 & $\# 1$ & 21.0 & $\# 6^{\mathrm{a}}$ & 42.0 & 650 & $6.0 \times 10^{-12}$ & $3.1 \times 10^{-12}$ & $\begin{array}{c}\text { SS in coarse layer } \\
\text { SS in fine layer }\end{array}$ \\
\hline Series 4 & $\# 1$ & 21.0 & $\# 7^{\mathrm{a}}$ & 21.0 & 1182 & $7.6 \times 10^{-13}$ & $2.3 \times 10^{-12}$ & $\begin{array}{l}\text { SS in coarse layer } \\
\text { SS in fine layer }\end{array}$ \\
\hline Parallel 1 & $\# 1$ & 39.5 & $\# 4$ & 39.5 & 68 & $6.0 \times 10^{-10}$ & $6.4 \times 10^{-10}$ & $\begin{array}{l}\text { SS in coarse layer } \\
\text { NSS }^{f} \text { in fine layer }\end{array}$ \\
\hline Parallel 2 & $\# 1$ & 39.5 & $\# 3^{\mathrm{a}}$ & 39.5 & 11 & $8.0 \times 10^{-10}$ & $6.9 \times 10^{-10}$ & $\begin{array}{l}\text { SS in coarse layer } \\
\text { NSS in fine layer }\end{array}$ \\
\hline Parallel 3 & $\# 1$ & 39.5 & $\# 2^{\mathrm{a}}$ & 39.5 & 3 & $1.1 \times 10^{-09}$ & $8.5 \times 10^{-10}$ & $\begin{array}{l}\text { SS in coarse layer } \\
\text { NSS in fine layer }\end{array}$ \\
\hline $\begin{array}{c}\text { Complex: } \\
\text { Disconnected Coarse }\end{array}$ & $\# 1$ & $26.0^{\mathrm{b}}$ & $\# 4$ & $53.0^{\mathrm{b}}$ & 68 & $6.4 \times 10^{-11}$ & $5.0 \times 10^{-11}$ & $\begin{array}{l}\text { SS in coarse layer } \\
\text { SS in fine layer }\end{array}$ \\
\hline 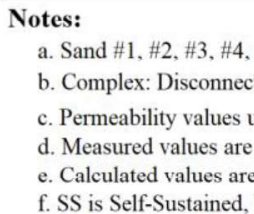 & $\begin{array}{l}\# 5, \# 6, \# 7 \\
\text { cted Coarse: } \\
\text { used for } \mathrm{kr} \\
\mathrm{e} \text { based on } \mathrm{tl} \\
\mathrm{e} \text { based on } \mathrm{n}\end{array}$ & $\begin{array}{l}\text { are from } \\
H_{1}=6.0 \\
\text { tio are fro }\end{array}$ & $\begin{array}{l}\text { le } 3.1 \text {. } \\
\mathrm{H}_{2}= \\
\text { "Meas }\end{array}$ & $\begin{array}{l}\mathrm{m}, \mathrm{H}_{3} \\
\text { sand } \mathrm{f} \\
\text { flux fo }\end{array}$ & $\begin{array}{l}3.0 \mathrm{~cm}, \mathrm{H}_{4}=6.5 \mathrm{cr} \\
\left.\mathrm{n}^{2}\right): \text { Cell" in Table } \\
\text { e treatment zone }\end{array}$ & $\begin{array}{l}=13.0 \mathrm{~cm}, \mathrm{~h}_{2} \\
\text { mouldering ( } \\
2.2 \text { below. }\end{array}$ & $\mathrm{m}, \mathrm{h}_{3}=16.0 \mathrm{~cm}$, & $=9.0 \mathrm{~cm}$. \\
\hline
\end{tabular}

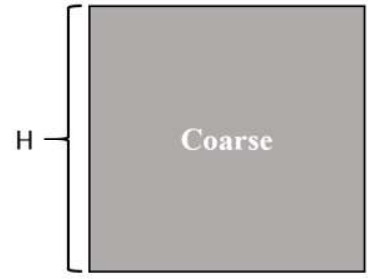

(a)

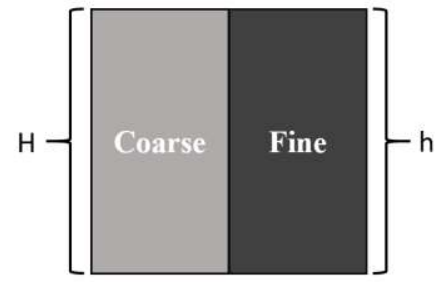

(c)

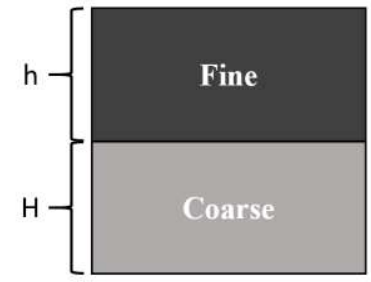

(b)

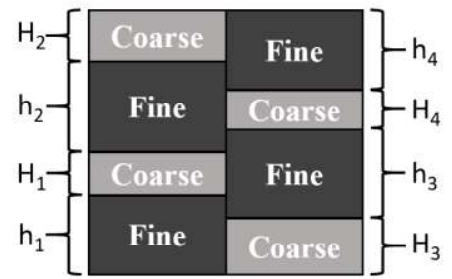

(d)

Figure 3.2: Experimental set-ups for treatment zones in all experiments: (a) Base Case, (b) Series 1-4, (c) Parallel 1-3 and (d) Complex: Disconnected Coarse. The height of each layer is summarized in Table 3.2. 
Two different smouldering columns were employed in this work (Figure 3.3). Column A was $63.0 \mathrm{~cm}$ tall and had a $16.0 \mathrm{~cm}$ inside diameter. Column B was $90.0 \mathrm{~cm}$ tall and had a $10.8 \mathrm{~cm}$ inside diameter. In addition, Column A employed smouldering travelling upwards while in Column B smouldering travelled downwards. The Base Case, the Parallel tests and Complex: Disconnected Coarse used Column A, while the Series tests used Column B. Column B was chosen for the Series tests because it can handle the much higher pressures generated during these experiments due to the fine layer spanning the column width and acting as a confining layer. The Base Case, the Parallel tests and Complex: Disconnected Coarse did not generate such backpressures and thus Column A was chosen, since it is simpler to operate and follows procedures used in many smouldering studies (e.g., Rashwan et al., 2016; Kinsman et al., 2017). Separate tests confirmed statistically identical results for Column A and B for otherwise identical tests, indicating that the choice of the column does not affect the behaviour of the smouldering reaction at the centerlines where temperatures, pressures, and reaction velocities were measured (see details in Appendix B). 


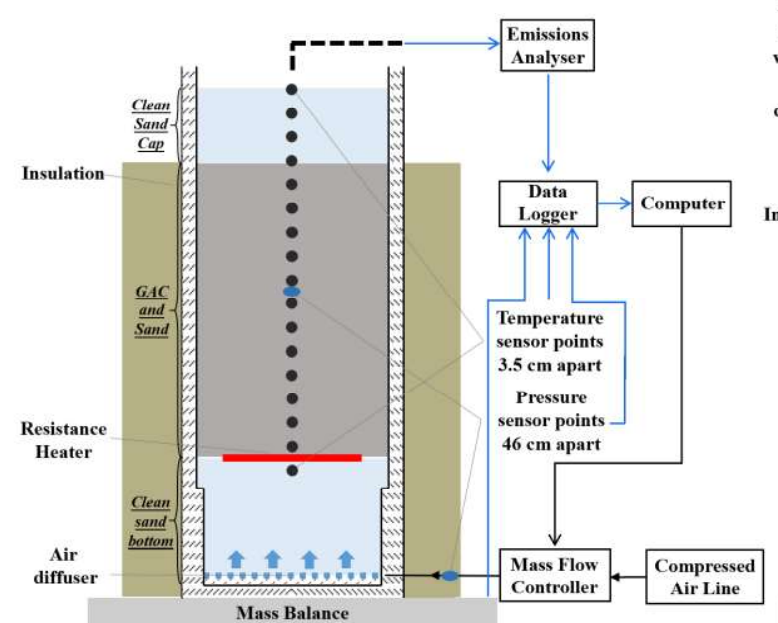

(a)

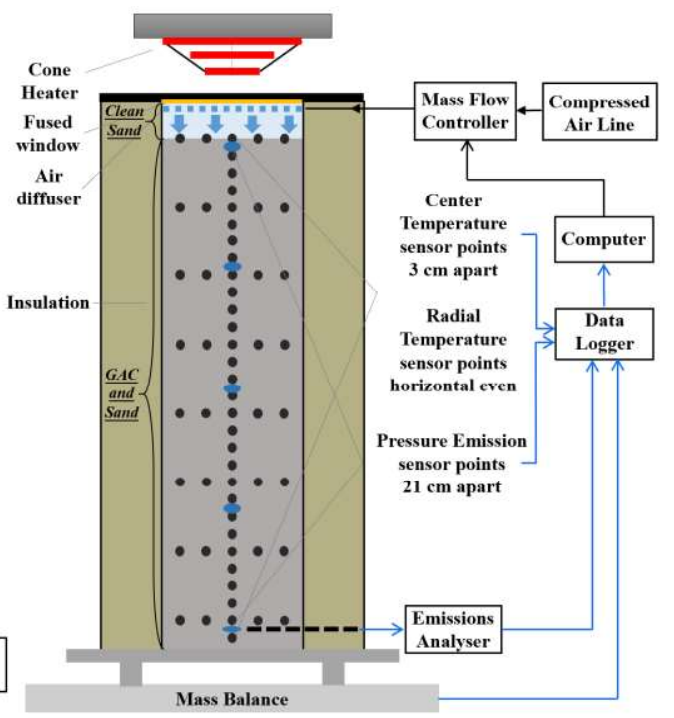

(b)

Figure 3.3: (a) Column A and (b) Column B set-ups for the smouldering tests.

Column A consisted of a stainless base assembly and a stainless column reactor (Figure 3.3a). A cable heater $(450 \mathrm{~W}, 120 \mathrm{~V}$, Watlow Ltd.) and an air injection manifold were housed in the base, where the cable heater was formed into a flat spiral coil to deliver a controlled amount of energy across the entire horizontal cross section. The column reactor was wrapped in insulation ( $5 \mathrm{~cm}$ thick mineral wool, McMaster Carr) to reduce heat losses (Switzer et al., 2009). Sixteen thermocouples (TCs) (K-type, KQIN-18U-6, Omega Ltd.) were inserted horizontally to track temperature at the center of the column at $3.5 \mathrm{~cm}$ intervals along the column height. Three pressure transducers (PTs) were employed (5 PSI, Model FPG, Honeywell) with two connected to copper tubing (designed to transmit the air pressure but dissipate the heat) located at a height of $46.0 \mathrm{~cm}$ and the other measuring the pressure in the air injection line. The top of the apparatus was open to the atmosphere.

Column B consisted of a structural support base, a cone heater $(5000 \mathrm{~W}, 240 \mathrm{~V}$, Fire Testing Technology Ltd.) and a stainless-steel column reactor assembly (Figure 3.3b). Insulation (5 $\mathrm{cm}$ thick Superwool Blanket, Morgan) outside the column was enclosed by a 
piece of aluminum sheet. The cone heater, set above the column, transmitted radiant heat through a fused quartz window (Esco Products Inc.). Thirty centerline TCs at $3.0 \mathrm{~cm}$ intervals, and eight sets of four radial TCs at $12.0 \mathrm{~cm}$ intervals, were employed. Four PTs (100 PSI, Type T2, Ashcroft Ltd.), attached to copper tubing, were recording pressures at $21.0 \mathrm{~cm}$ intervals.

In each case, the apparatus rested on a balance (KCC150, Metler Toledo) to provide realtime mass loss data. In addition, the combustion emissions were sampled by a gas analyzer (MGA 3000C, ADC) for the real-time determination of the volume fraction of oxygen, carbon monoxide and carbon dioxide. Data from TCs, PTs, mass balance and gas analyzer were recorded every two seconds by data loggers (Multifunction Switch/Measure Unit 34980A, Agilent Technologies) and personal computers.

Nine smouldering experiments were packed as described in Table 3.2 and Figure 3.2. In all experiments, a clean sand layer $(20.5 \mathrm{~cm}$ in Column A, $1.0 \mathrm{~cm}$ in Column B) near the ignition point was used to cover the heater and help evenly distribute air. Also, after packing of the contaminated sand, another clean sand layer filled the rest of the column to ensure the pack cannot fluidize. Each packed column was subjected to cold air injection at several known air fluxes before smouldering (GAC present) and after smouldering when the system had completely cooled (GAC absent). In combination with the pressure transducers and known ambient pressure at the outlet, this allowed the in situ determination of intrinsic permeability values of interest. When using pressures within a single layer, in situ permeability of a specific sand was determined, reported as "Measured sand $\mathrm{k}\left(\mathrm{m}^{2}\right)$ : In situ" in Table 3.1. When using a pair of pressures that bound the entire treatment zone, this provided an equivalent permeability measurement of the ensemble of layers (Figure 3.2); 
these measurements, conducted post-treatment, are reported as "Treatment zone $\mathrm{k}\left(\mathrm{m}^{2}\right)$ : Measured" in Table 3.2. These were compared to the theoretical (calculated) equivalent pack permeability reported in Table 3.2 as "Treatment zone $\mathrm{k}\left(\mathrm{m}^{2}\right)$ : Calculated" and calculated as described next.

For Series 1-4, the permeability of the treatment zone was dominated by the low permeability layer. An equivalent permeability was the harmonic mean of the value for the layers (Leonards, 1962):

$$
k=\frac{\sum_{i=1}^{M} b_{i}}{\sum_{i=1}^{M} b_{i} / k_{i}}
$$

where $k$ is equivalent permeability $\left(\mathrm{m}^{2}\right), k_{i}$ is the permeability $\left(\mathrm{m}^{2}\right)$ of layer $\mathrm{i}$, and $b_{i}(\mathrm{~m})$ is the thickness of the layer i. Table 3.2 reveals that these calculations provide similar values to those directly measured in the packed columns. The table also reveals that the permeability contrast between the coarse and fine layers in series increased to three orders of magnitude.

For Parallel 1-3, the permeability of the treatment zone was dominated by the high permeability layer in the mixture. The equivalent permeability was based on the arithmetic mean (Leonards, 1962):

$$
k=\frac{\sum_{i=1}^{M} b_{i} k_{i}}{\sum_{i=1}^{M} b_{i}}
$$

Table 3.2 reveals that in Parallel 1-3, the equivalent permeability values estimated via Equation 2 are close to those measured in situ in the column. The table further shows that the contrast in permeability between parallel layers increased to a maximum of 68 . 
For the Complex: Disconnected Coarse, which combined layers in series and in parallel in the form of a checkerboard, both high and low permeability layers impacted the equivalent permeability of the pack; therefore, the geometric mean was used (Selvadurai and Selvadurai, 2014):

$$
k=\sqrt[M]{\prod_{i=1}^{M} k_{i}}
$$

Table 3.2 reveals that in the Complex: Disconnected Coarse, the equivalent permeability value estimated via Equation 3 is close to the direct measurement in the column, in which the contrast in permeability between layers was 68 .

The smouldering tests employed well-established experimental procedures (Switzer et al., 2009; Pironi et al., 2011; Yermán et al., 2015). The ignition procedure involved starting the heater and leaving it on until $\mathrm{TC} 1$ in the treatment zone reached $400{ }^{\circ} \mathrm{C}$ in Column A ( $1 \mathrm{~cm}$ above the clean sand), and $450{ }^{\circ} \mathrm{C}$ in Column B (at the interface between the treatment zone and clean sand). Air injection was then initiated, ignition of smouldering was confirmed by a temperature spike, and the heater was turned off after the second thermocouple in the treatment zone peaked. The self-sustained propagation of the reaction along the column was monitored until it reached the end of the treatment zone, at which time the smouldering naturally terminated (no fuel). Air injection was maintained until the system returned to ambient temperatures.

The smouldering reaction velocity and the average reaction temperature were calculated according to standard procedures, whose uncertainty is represented by $95 \%$ confidence intervals (Pironi et al., 2009). Additionally, in Parallel 1-3, the distribution of the injected air amongst the layers (at ambient temperatures) was calculated via Darcy's Law by solving 
three equations for three unknowns (assuming one-dimensional vertical airflow and uniform pressure distribution across the horizontal cross-section within each layer):

$$
\begin{gathered}
q_{\text {total }}=\frac{q_{1}+q_{2}}{2} \\
q_{1}=\frac{k_{\text {Fine }}\left(P_{1}-d p_{0-1}-P_{2}\right)}{\mu l} \\
q_{2}=\frac{k_{\text {Coarse }}\left(P_{1}-d p_{0-1}-P_{3}\right)}{\mu l}
\end{gathered}
$$

where $q_{\text {total }}$ is the fixed injection Darcy air flux $(2.5 \mathrm{~cm} / \mathrm{s}), q_{1}$ and $q_{2}$ are the unknown fine and coarse layer air fluxes $(\mathrm{cm} / \mathrm{s})$, respectively; $P_{1}, P_{2}$ and $P_{3}$ are pressure readings (Pa) from pressure transducers PT1 (in the air injection line), PT2 (in the fine layer) and PT3 (in the coarse layer); $d p_{0-1}$ is the unknown pressure loss $(\mathrm{Pa})$ due to the clean sand layer between the injection location and the treatment zone; $k_{\text {Fine }}$ and $k_{\text {Coarse }}$ are permeabilities $\left(\mathrm{m}^{2}\right)$ of the fine and coarse layers, respectively, measured from the permeability cell (Table 3.2$) ; \mu$ is the ambient viscosity $(\mathrm{kg} / \mathrm{m} / \mathrm{s})$ of air; $l$ is the height $(\mathrm{m})$ of PT2 and PT3 above the upper surface of the clean bottom sand layer.

\subsubsection{Numerical Modelling}

MacPhee et al. (2012), Hasan et al. (2015) and Solinger et al. (2019) presented the development and validation of a two-dimensional (2-D) in situ smouldering model (ISSM) to predict the propagation of a smouldering reaction in heterogeneous contaminated soil. The model coupled a finite difference, multiphase flow numerical model (Gerhard and Kueper, 2003a, 2003b, 2003c) with a combustion reaction evolution model (Richards, 1990, 1995). The model was demonstrated to reliably predict the propagation of the smouldering reaction under multidimensional air injection flow fields in homogeneous soil, including 
reaction extinction in regions where air flux was too low to support a self-sustained reaction (Hasan et al., 2015). It was also shown to predict complex channelling of the reaction that might occur in highly heterogeneous porous media, although such cases were numerical predictions and not validated against experiments (Solinger et al., 2019). The model did not consider energy balance or heat transfer and was thus primarily an engineering tool for exploring the influence of air flux, and related Darcy parameters such as pressure and permeability, on reaction propagation (MacPhee et al., 2012). The ISSM was employed in this work to simulate and help explain experimental results of Parallel 1-3 and Complex: Disconnected Coarse. The underlying principles of the ISSM are briefly summarized in Appendix E; refer to MacPhee et al. (2012), Hasan et al. (2015) and Solinger et al. (2019) for more details.

As shown in Figure 3.4, a $16.0 \mathrm{~cm}$ wide $\times 71.0 \mathrm{~cm}$ tall model domain (including a $20.5 \mathrm{~cm}$ bottom clean sand layer, a $39.5 \mathrm{~cm}$ treatment zone, a $10.5 \mathrm{~cm}$ clean sand cap layer and a $0.5 \mathrm{~cm}$ bottom plenum for the air distribution in the model) was designed to approximate a 2-D vertical cross-section of the Parallel 1-3 and two Complex cases, in which an additional scenario Complex: Connected Coarse was also simulated to compare with Complex: Disconnected Coarse.

A maximum time step of 5 seconds was selected because this value was previously demonstrated to retain accuracy while preventing excessive run times (MacPhee et al., 2012). A nodal discretization of $0.5 \mathrm{~cm} \times 0.5 \mathrm{~cm}$ was selected, for a total of 4544 nodes in the domain (32 nodes along the $\mathrm{x}$ axis, 142 nodes along the $\mathrm{y}$ axis). No-flow conditions were applied to the left and right boundaries to simulate the column walls. The $0.5 \mathrm{~cm}$ deep plenum was placed in the bottom of the domain as a single row of nodes and was 
approximated with a constant air flux of $2.5 \mathrm{~cm} / \mathrm{s}$, whose permeability was assigned as 1.0 $\times 10^{-10} \mathrm{~m}^{2}$. The top row of nodes was assigned a free-exit boundary, where the air pressure was zero. The ignition source was specified as $15.5 \mathrm{~cm}$ long $\times 0.5 \mathrm{~cm}$ high initial ellipse at the center of the interface between bottom clean sand nodes and treatment zone nodes as the time zero smouldering reaction. The distribution of permeability followed the experimental set-up and data measured from the permeability cell as shown in Tables 3.1 and 3.2. The nodes inside the treatment zone were assigned a small fuel concentration to represent the negligible impact the GAC had on effective permeability (Table 3.1). Run time for each simulation was approximately 60 minutes using an Intel Core Processor i73930K CPU with 64 GB RAM.

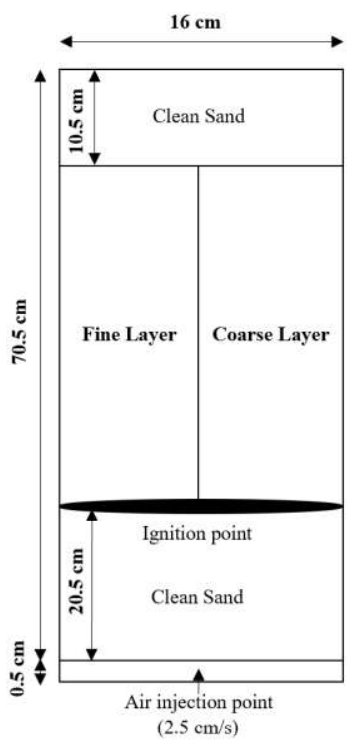

(a)

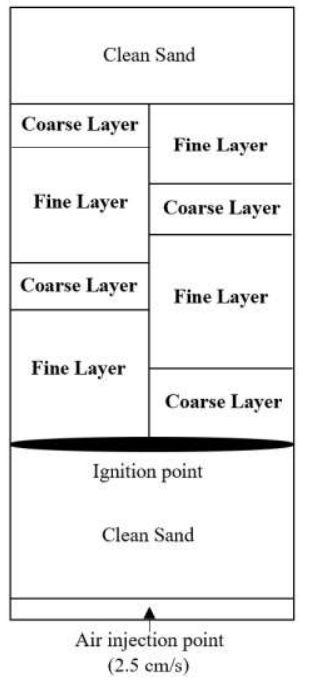

(b)

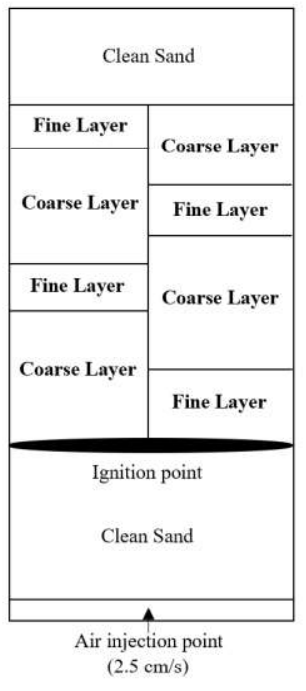

(c)

Figure 3.4: Illustration of Model set-ups of (a) Parallel 1-3, (b) Complex: Disconnected Coarse and (c) Complex: Connected Coarse simulations. 


\subsection{Results and Discussion}

\subsubsection{Base Case}

Figure 3.5 provides the temperature, pressure, emission and mass loss rate profiles for the Base Case. It illustrates that upon initiating the airflow, a temperature spike was observed that indicated the onset of the smouldering reaction (i.e., ignition). The heater was turned off a short time later, after which the reaction was self-sustained as evidenced by the succession of crossing temperature profiles with consistent peak temperatures (Pironi et al., 2009). Slight variations in the peak temperatures were expected due to small heterogeneities in the contaminated mixture (e.g., local variations in the GAC concentration and particle size) from the mixing and loading process. The average peak temperature was $774 \pm 31^{\circ} \mathrm{C}$ and the average reaction velocity was $3.8 \pm 0.2 \mathrm{~mm} / \mathrm{min}$. The pressure signals provided the gradients associated with the airflow and provided data for quantifying the effective permeability of the treatment zone. The pressure signals associated with smouldering, rarely measured in the literature, provide a wealth of valuable information about tracking and interpreting smouldering that are explored in detail in Appendix D and parts of which have been submitted for publication as a separate manuscript. 


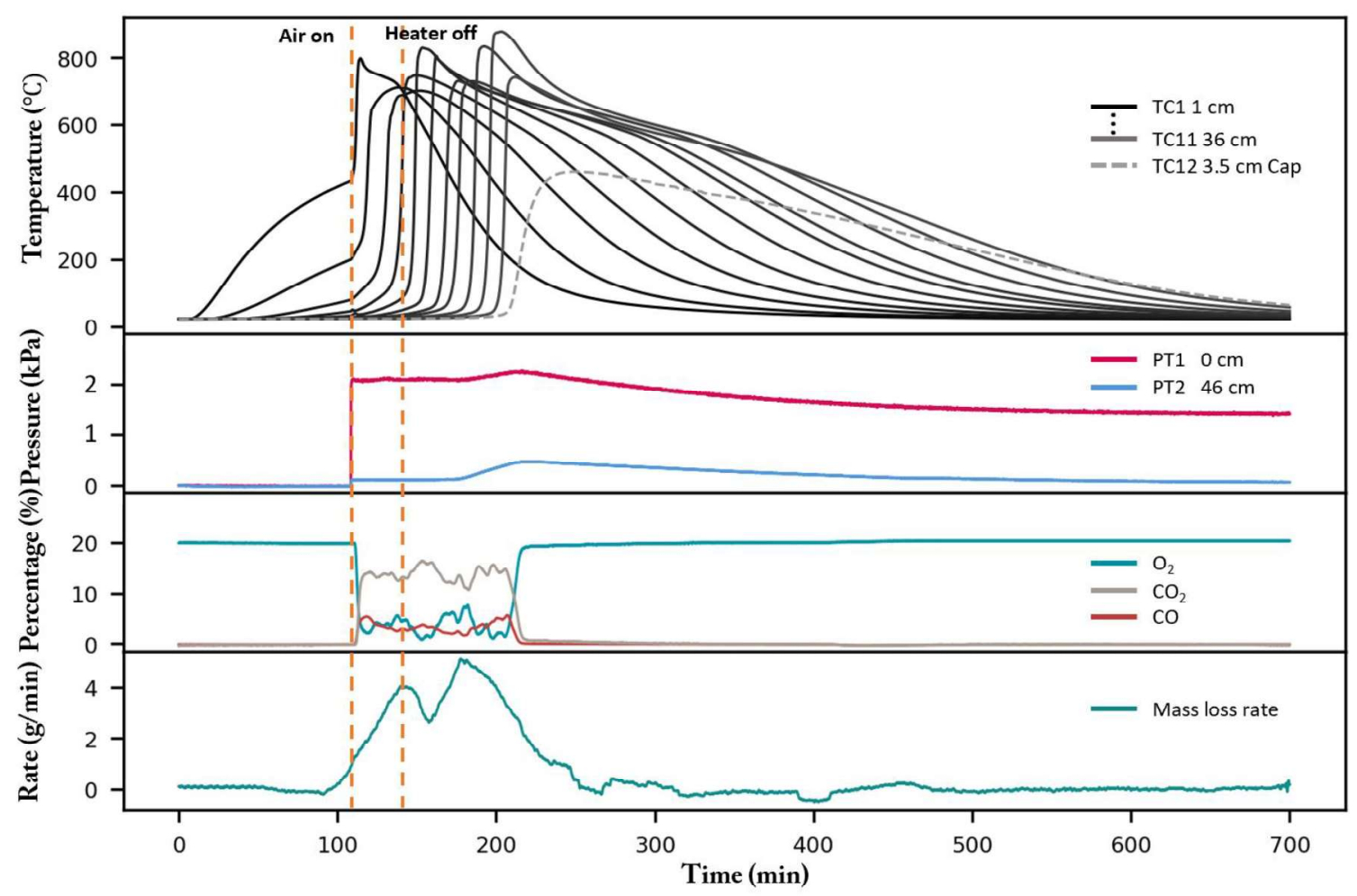

Figure 3.5: Temperature, pressure, emission and mass loss rate profiles for Base Case. $\mathrm{CO}, \mathrm{CO}_{2}$ and $\mathrm{O}_{2}$ in the emissions are also shown in Figure 3.5, revealing that, as expected, $\mathrm{O}_{2}$ content plunged and $\mathrm{CO}$ and $\mathrm{CO}_{2}$ contents spiked at the same time the temperature indicated the smouldering reaction started. At the same time, the mass loss rate increased since the contaminant was being oxidized by smouldering. Mass loss rate related to the GAC removal rate and emissions were relatively steady through the smouldering period, although some variations due to minor heterogeneities within the pack are typical (Switzer et al., 2009, 2014; Rashwan et al., 2016). Once smouldering was complete in the column, pressure decreased, emissions returned to ambient and mass loss rate reduced, coinciding with the last TC peak, and the system cooled. 


\subsubsection{Series 1-4}

Series 1 with a single homogeneous fine layer showed that smouldering was still robust when the permeability of the porous medium was significantly decreased ( $1 \%$ of Base Case), with a similar average peak temperature $\left(758 \pm 22^{\circ} \mathrm{C}\right)$ but with an approximately $25 \%$ reduction in reaction velocity $(2.8 \pm 0.2 \mathrm{~mm} / \mathrm{min})$ (Figure $3.6 \mathrm{a})$. The change in reaction velocity is discussed below with other Series tests. In general, as expected, pressure drop across the column in this case $(\Delta \mathrm{P} \approx 60 \mathrm{kPa}$; Figure. 3.6a) was much higher than the Base Case ( $\Delta \mathrm{P} \approx 2 \mathrm{kPa}$; Figure 3.5), yet this yielded no adverse effect on the smouldering behaviour since the GAC concentration and air flux were unchanged.

(a)

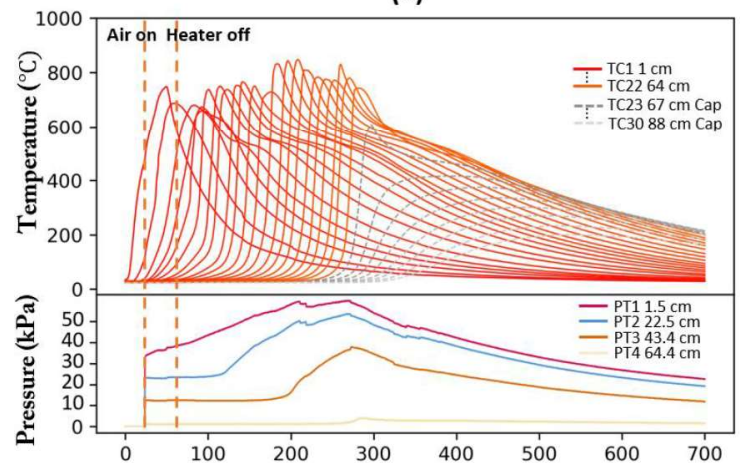

(c)

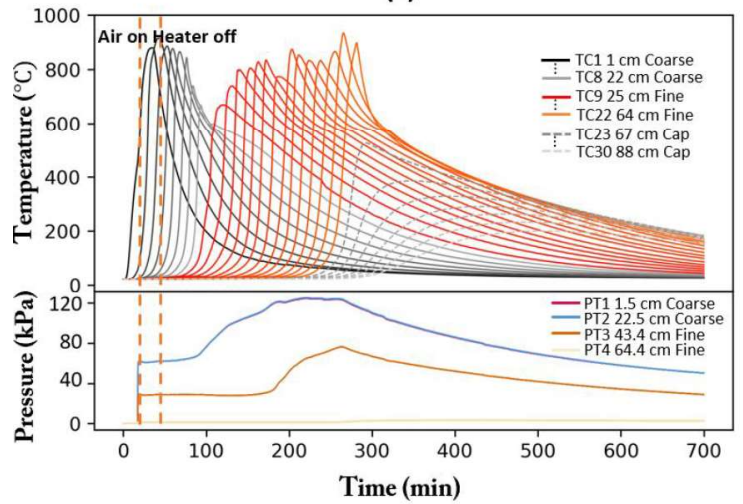

(b)

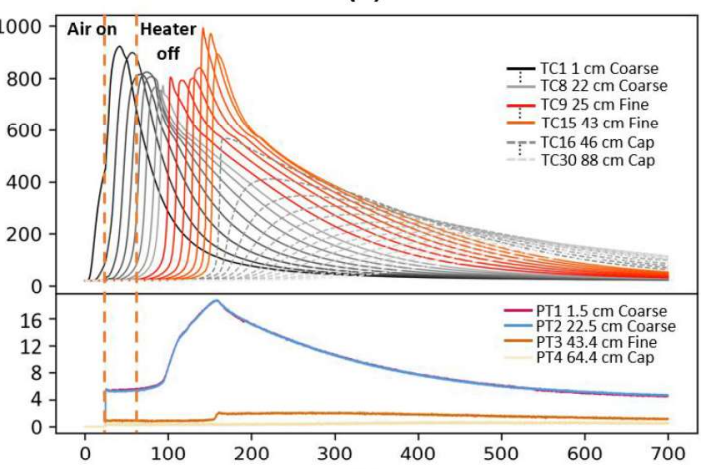

(d)

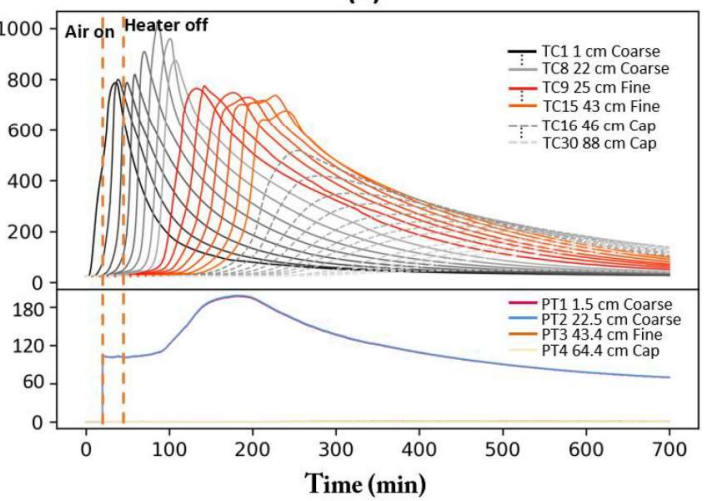

Figure 3.6: Temperature and pressure profiles for Series 1-4: (a) Series 1, (b) Series 2, (c) Series 3 and (d) Series 4. Black temperature curves are for TCs located in the coarse layer while red curves are for TCs located in the fine layer. Note that, while the temperature axes are the same, the pressure axes have different scales in the four figures. 
Figure 3.6 also reveals that the occurrence of a coarse layer and fine layer in series was no barrier to the self-sustained smouldering. All three tests (Series 2-4) show self-sustained smouldering propagating from the coarse layer into the fine layer regardless of the fine layer permeability (at least down to $1.1 \times 10^{-12} \mathrm{~m}^{2}$, representing a 1000 -fold permeability contrast). These conclusions were confirmed when excavation revealed no GAC remaining in any of the layers. This is the first laboratory evidence that smouldering will readily transit across distinct, high-contrast permeability boundaries. As expected, the pressure gradient across the column increased proportionally as the equivalent permeability of the treatment zone decreased. The pressure drop increased from $\sim 20 \mathrm{kPa}$ in Series 2 to $\sim 200 \mathrm{kPa}$ in Series 4, which represented the upper limit the apparatus can endure.

Figure 3.7 summarizes the peak temperature and reaction velocity of self-sustained smouldering in the individual layers in Series 1-4. It reveals that permeability did not affect the peak temperature of smouldering; all were observed to be above $700{ }^{\circ} \mathrm{C}$ and the variations observed were likely random effects of minor GAC inhomogeneity. The statistical F-test revealed that the average peak temperatures in all layers in Base Case and Series 1-3 showed no statistically significant difference at the 0.01 significance level, whereas the average peak temperature of the fine layer in Series 4 was statistically lower. Moreover, the figure also shows that the reaction velocity slightly reduced as the permeability of the fine layer decreased. Since air flux affects smouldering peak temperature and reaction velocity (Zanoni et al., 2019), it is hypothesized that (a) the observed reduction of average peak temperature in Series 4 and decreasing reaction velocity may be due to increased side wall effects, where a small but increasing fraction of the air preferentially travelled along the sand/wall boundary in low permeability materials, 
reducing the air flux propagating the reaction along the centerline, and/or (b) the heat transfer processes (conduction, convection, storage, losses) varied slightly as sands became finer, affecting the reaction propagation. Note that neither the average peak temperature nor reaction velocity in the coarse layer was significantly affected by the presence of a fine layer in any of the Series tests.

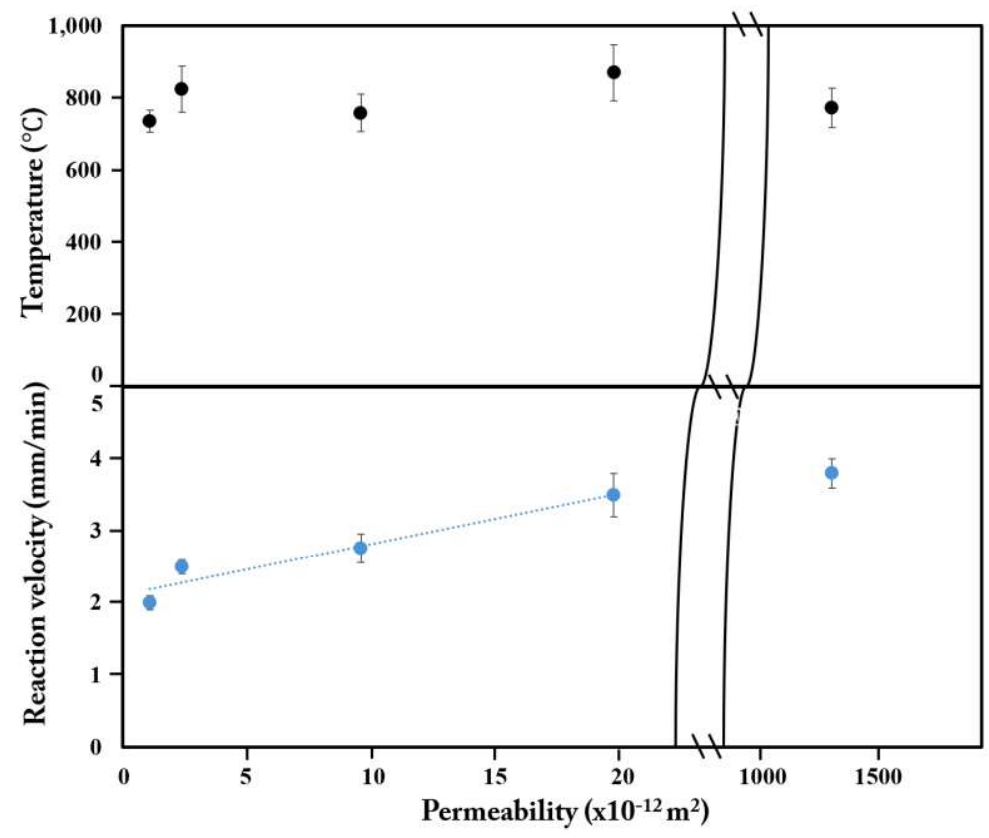

Figure 3.7: Average peak temperature and self-sustained smouldering velocity for each individual layer, plotted with its measured permeability within Series 1-4. Uncertainty is represented by $95 \%$ confidence intervals.

\subsubsection{Parallel 1-3}

Parallel 1, in which the permeability difference between the parallel layers was 1.5 orders of magnitude $\left(1.3 \times 10^{-9} \mathrm{~m}^{2}\right.$ and $\left.1.9 \times 10^{-11} \mathrm{~m}^{2}\right)$, resulted in self-sustained smouldering in the coarse layer (average peak temperature $713 \pm 42{ }^{\circ} \mathrm{C}$ ) and non-sustained smouldering in the fine layer (Figure 3.8). The fine layer thermocouples did not show the sharp temperature spikes and crossing profiles associated with the heat generation process in smouldering, but rather the rounded and coincident profiles associated with only heat 
transfer processes (Salman et al., 2015; Zanoni et al., 2017). The temperature profiles reveals that the elevated temperatures observed in the fine layer almost exclusively resulted from lateral, conductive heat transfer from the smouldered coarse layer; in other words, thermal coupling caused some energy losses from the reaction in the coarse layer to heat the adjacent fine layer, as predicted by Akkutlu and Yortsos (2015). The pressure, emissions, and mass loss rate data all support that smouldering was only occurring in the coarse layer, as their signals diminished quickly after smouldering of that layer was complete. Confirmation that smouldering did not occur in the fine layer was provided by observing GAC remaining in the fine layer during excavation (see photos in Appendix C).

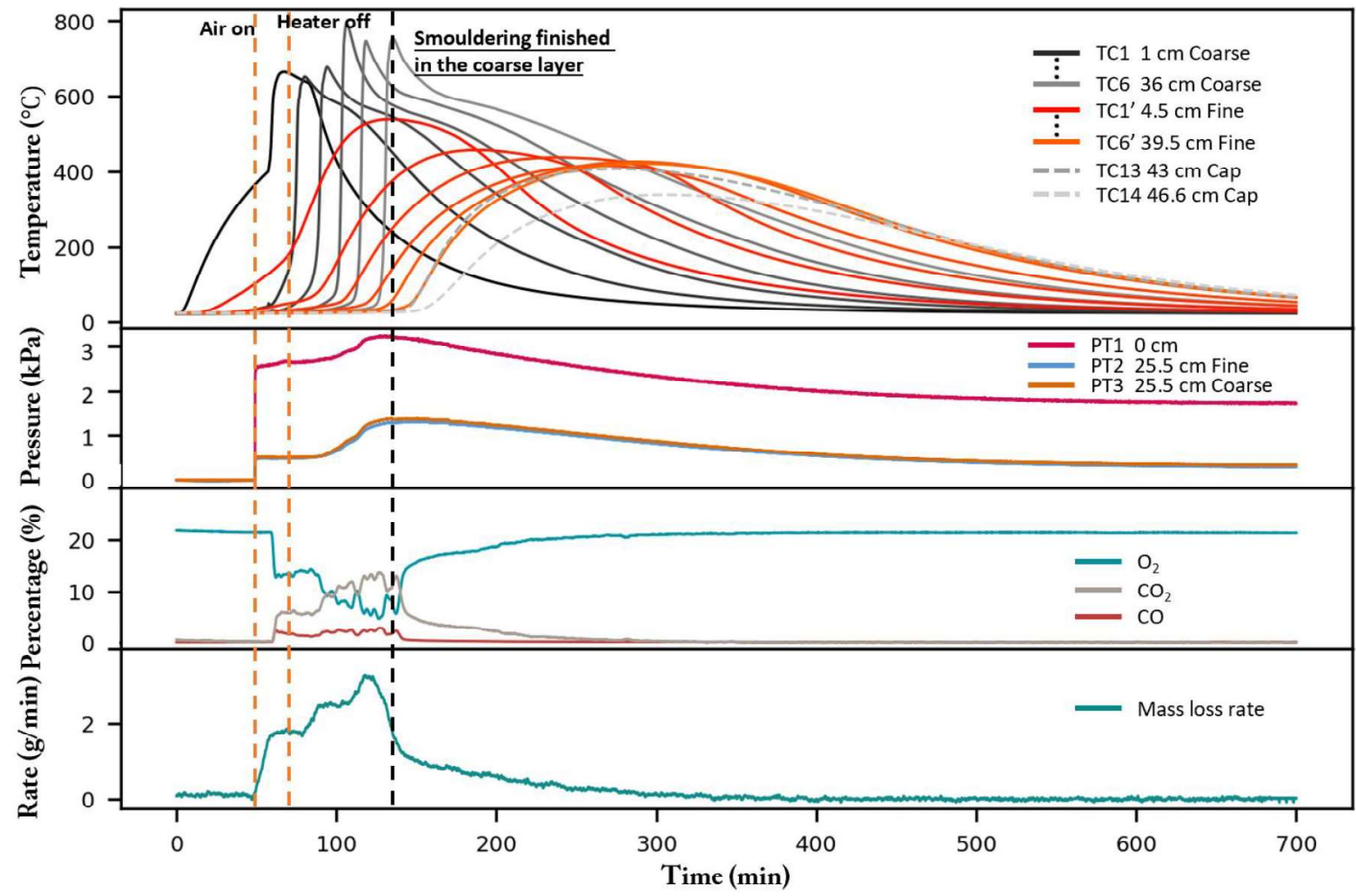

Figure 3.8: Temperature, pressure, emission and mass loss rate profiles for Parallel 1.

The average smouldering reaction velocity in the coarse layer was $5.0 \pm 0.3 \mathrm{~mm} / \mathrm{min}$, which was faster than the velocity through a layer of equal permeability in the Base Case 
( $3.8 \pm 0.2 \mathrm{~mm} / \mathrm{min}$ ), even though air was injected into the column with the same Darcy flux $(2.5 \mathrm{~cm} / \mathrm{s})$. Based on the methodology outlined in Section 3.2.2, the distribution of air fluxes was calculated as $4.91 \mathrm{~cm} / \mathrm{s}$ and $0.09 \mathrm{~cm} / \mathrm{s}$ in the coarse and fine layers, respectively. The higher proportion of air traversing the coarse layer, leading to a higher air flux in that layer, explains the higher smouldering propagation velocity relative to the Base Case. Moreover, these calculations suggest that the lack of smouldering in the fine layer was mainly due to the limited air flux, since other work has indicated that smouldering is rarely self-sustained when the local air flux in a laboratory experiment is below $0.5 \mathrm{~cm} / \mathrm{s}$ (MacPhee et al., 2012; Hasan et al., 2015; Solinger et al., 2019).

Parallel 2 employed a one order of magnitude difference in permeability $\left(1.3 \times 10^{-9} \mathrm{~m}^{2}\right.$ and $1.2 \times 10^{-10} \mathrm{~m}^{2}$ ). Smouldering in the coarse layer was self-sustained, with an average peak temperature of $696 \pm 62{ }^{\circ} \mathrm{C}$ and the average reaction velocity of $5.7 \pm 0.4 \mathrm{~mm} / \mathrm{min}$ (Figure. 3.9). Smouldering at the base of the fine layer ignited after ignition occurred in the coarse layer (TC1 in fine layer rose semi-sharply to $640{ }^{\circ} \mathrm{C}$ ). However, beyond this time the reaction clearly died as the rest of TCs in the fine layer showed only heat transfer characteristics. This interpretation is supported by the pressure, emissions, and mass loss data; moreover, GAC was found in the upper half of the fine layer upon excavation, whereas most of the GAC was eliminated near the base of the fine zone (close to the two bottom-most TCs) (see Appendix C). 


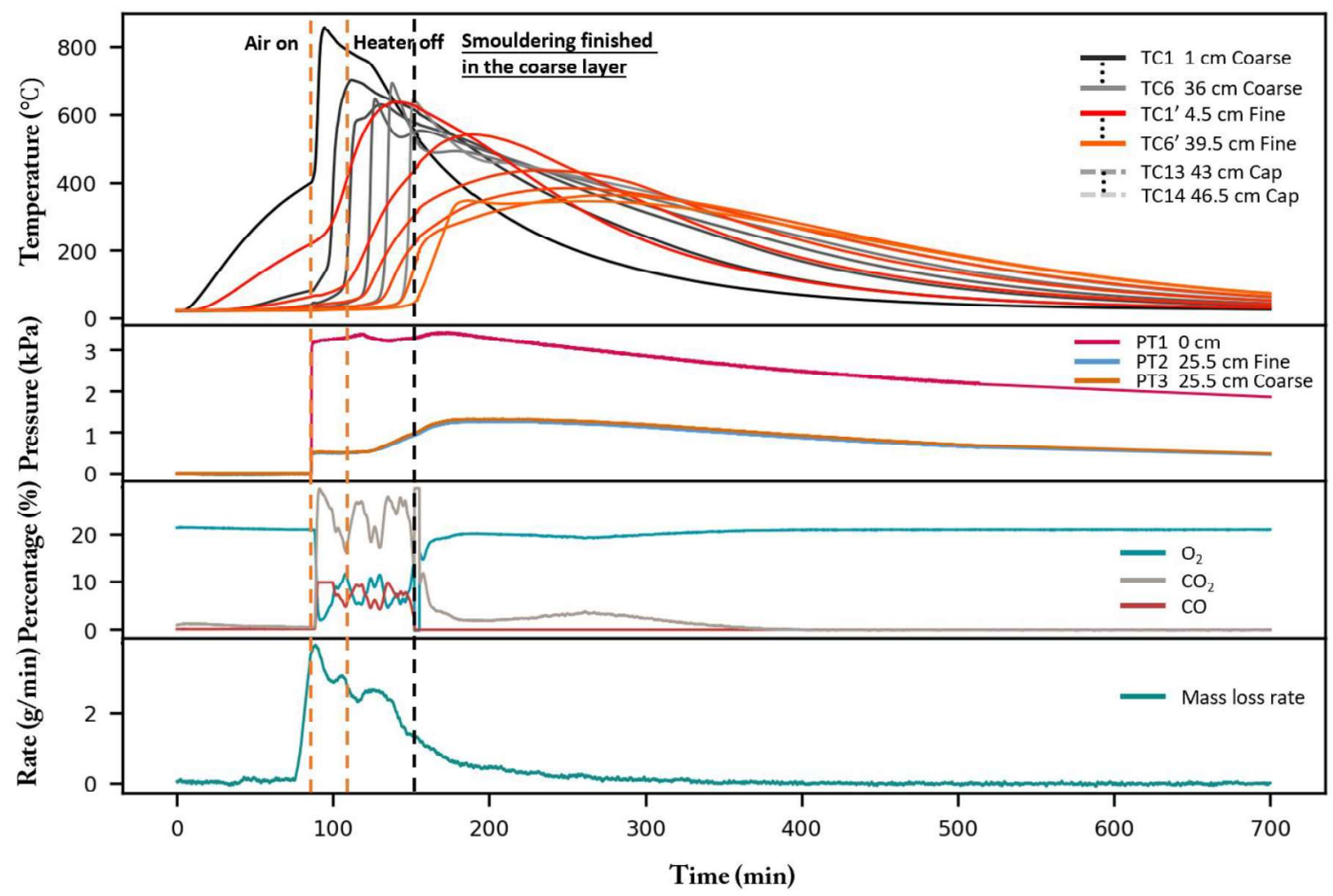

Figure 3.9: Temperature, pressure, emission and mass loss rate profiles for Parallel 2.

Calculations reveal that the air flux was $0.49 \mathrm{~cm} / \mathrm{s}$ in the fine layer and $4.51 \mathrm{~cm} / \mathrm{s}$ in the coarse layer. It is interesting that although a higher air flux traversed the coarse layer in Parallel $1(4.91 \mathrm{~cm} / \mathrm{s})$, the coarse layer smouldering velocity in that experiment was lower $(5.0 \pm 0.3 \mathrm{~cm} / \mathrm{s})$. This observation agrees with the prediction of Akkutlu and Yortsos (2005), in which thermal coupling slowed down the reaction in the coarse layer. In Parallel 2 , the smouldering ignition in the fine layer decreased lateral conductive heat transfer between the layers compared to Parallel 1. This resulted in more energy remaining within the coarse layer to contribute to its self-sustained smouldering; this observation is also supported by higher peak temperatures at the base of the coarse layer in Parallel 2. Moreover, since $0.49 \mathrm{~cm} / \mathrm{s}$ was very close to the air flux extinction threshold, it was not surprising that ignition occurred but that smouldering was not be sustained in the fine layer. 
Parallel 3 entailed a permeability difference of a half-order of magnitude $\left(1.3 \times 10^{-9} \mathrm{~m}^{2}\right.$ and $4.5 \times 10^{-10} \mathrm{~m}^{2}$ ). As with the other Parallel cases, smouldering in the coarse layer was robust (Figure 3.10), with an average peak temperature of $811 \pm 45^{\circ} \mathrm{C}$ and smouldering velocity of $4.5 \pm 0.2 \mathrm{~mm} / \mathrm{min}$. However, in this case a self-sustained smouldering reaction clearly ignited and propagated in the fine layer as well. Smouldering ignition in the fine layer occurred around the same time as ignition in the coarse layer. Average peak temperature in the fine layer was $774 \pm 27^{\circ} \mathrm{C}$ over the first three TCs, which represented the first $18.5 \mathrm{~cm}$ out of $39.5 \mathrm{~cm}$ treatment zone height, and smouldering velocity was $3.4 \pm 0.2 \mathrm{~mm} / \mathrm{min}$. However after approximately $19 \mathrm{~cm}$ of propagation, the smouldering reaction was observed to gradually weaken with reducing peak temperatures and no evidence for smouldering was observed at the final thermocouple in the fine layer. During the excavation of the fine layer, the bottom was clean whereas GAC was found at the top, supporting the conclusion that the reaction started and propagated but extinguished near the top of the fine layer (see Appendix C). 


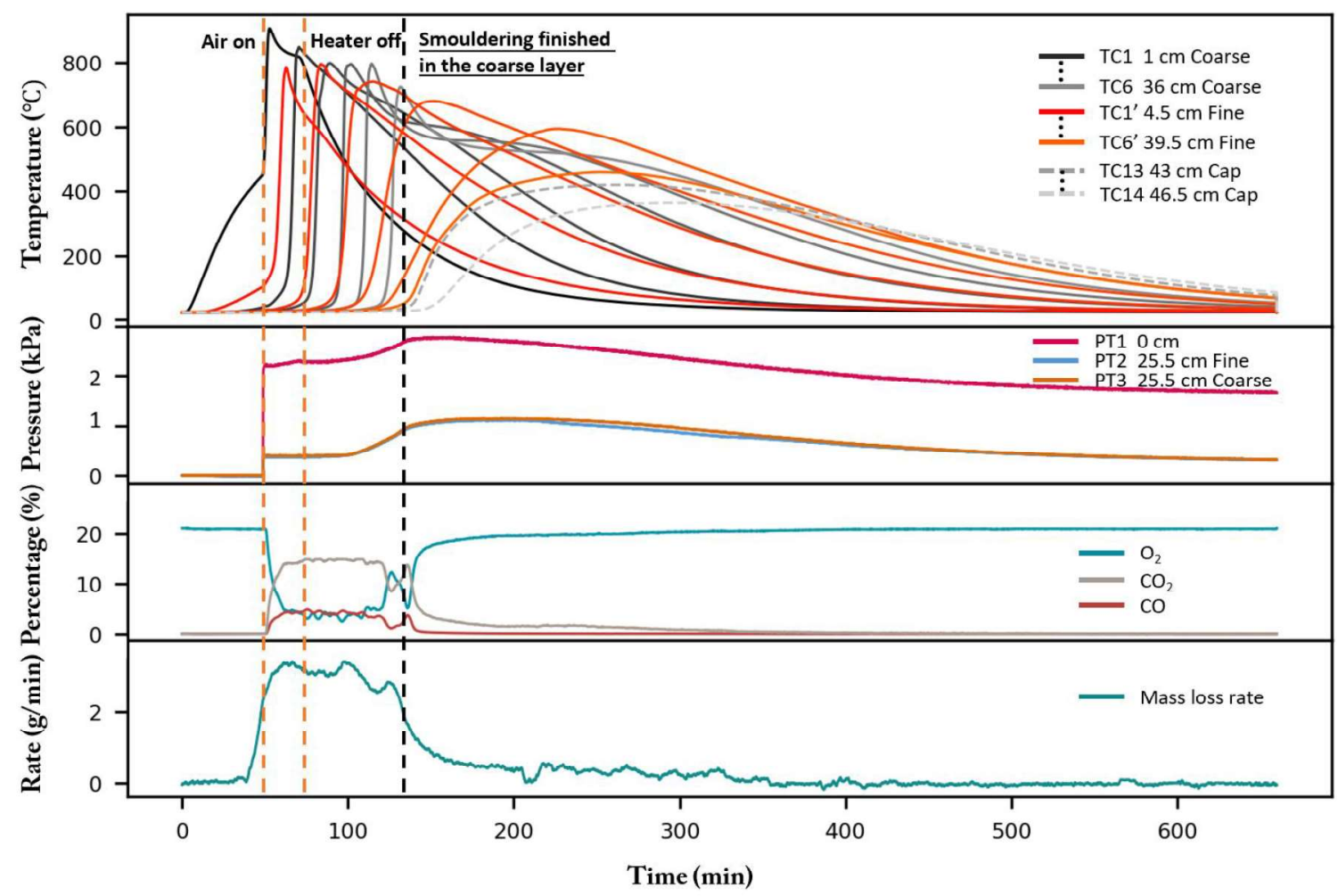

Figure 3.10: Temperature, pressure, emission and mass loss rate profiles for Parallel 3.

Calculated air flux in the fine layer was $1.29 \mathrm{~cm} / \mathrm{s}$ and in the coarse layer was $3.71 \mathrm{~cm} / \mathrm{s}$.

The existence of air flux in the fine layer well above the threshold for smouldering, the strong ignition, and the propagation of a self-sustained reaction suggests that the air flux was not the main factor quenching the fine layer smouldering in this case. Instead, it is hypothesized that lateral conductive heat transfer from the fine to coarse layers at the late time caused the reaction to die (Akkutlu and Yortsos, 2015). As shown in Figure 3.10, weakened smouldering in the fine layer was coincident with smouldering reaching completion in the coarse layer. The data suggest that after this time, the coarse layer acted as a lateral energy sink for the fine zone, drawing heat away from the fine layer by (i) lateral conduction across the interface, and then (ii) upward convection in the coarse layer. This is evidenced by the slower cooling rate of the coarse zone here compared to Parallel 
1 and 2. Therefore, excessive heat losses from the smouldering fine layer to the treated coarse layer likely led to the smouldering extinction observed in the fine layer. This hypothesis is also supported by the analytical modelling of Akkutlu and Yortsos (2005), which predicted that thermal coupling between a smouldering reaction adjacent to a nonsmouldering layer may negatively affect the reaction, such as reducing its reaction velocity and even leading to extinction under certain conditions.

\subsubsection{Complex: Disconnected Coarse}

The Complex: Disconnected Coarse employed multiple short layers in the form of a checkerboard, using a permeability difference of 1.5 orders of magnitude (sands were the same as Parallel $1,1.3 \times 10^{-9} \mathrm{~m}^{2}$ and $1.9 \times 10^{-11} \mathrm{~m}^{2}$ ), where disconnected coarse layers exist in the fine mixture (i.e., the fine sand dominated in the whole mixture). The objective here was to combine the fine and coarse layers both in series and parallel, disconnecting

the coarse layers, reducing the layers' lengths relative to the length of the system and ensuring no continuous high permeability path from inlet to outlet. In contrast to Parallel 1, self-sustained smouldering in all fine layers occurred in this case (Figure 3.11), with an average peak temperature of $826 \pm 55^{\circ} \mathrm{C}$. An average reaction velocity of $2.0 \pm 0.4$ $\mathrm{mm} / \mathrm{min}$ was observed in the fine layers, showing that air flux through the fine layers was sufficient to successfully sustain smouldering, unlike in Parallel 1. This is in addition to self-sustained smouldering in all the coarse layers (average peak temperature of $\left.774 \pm 76^{\circ} \mathrm{C}\right)$; therefore, in this case, the entire treatment zone was successfully smouldered. 


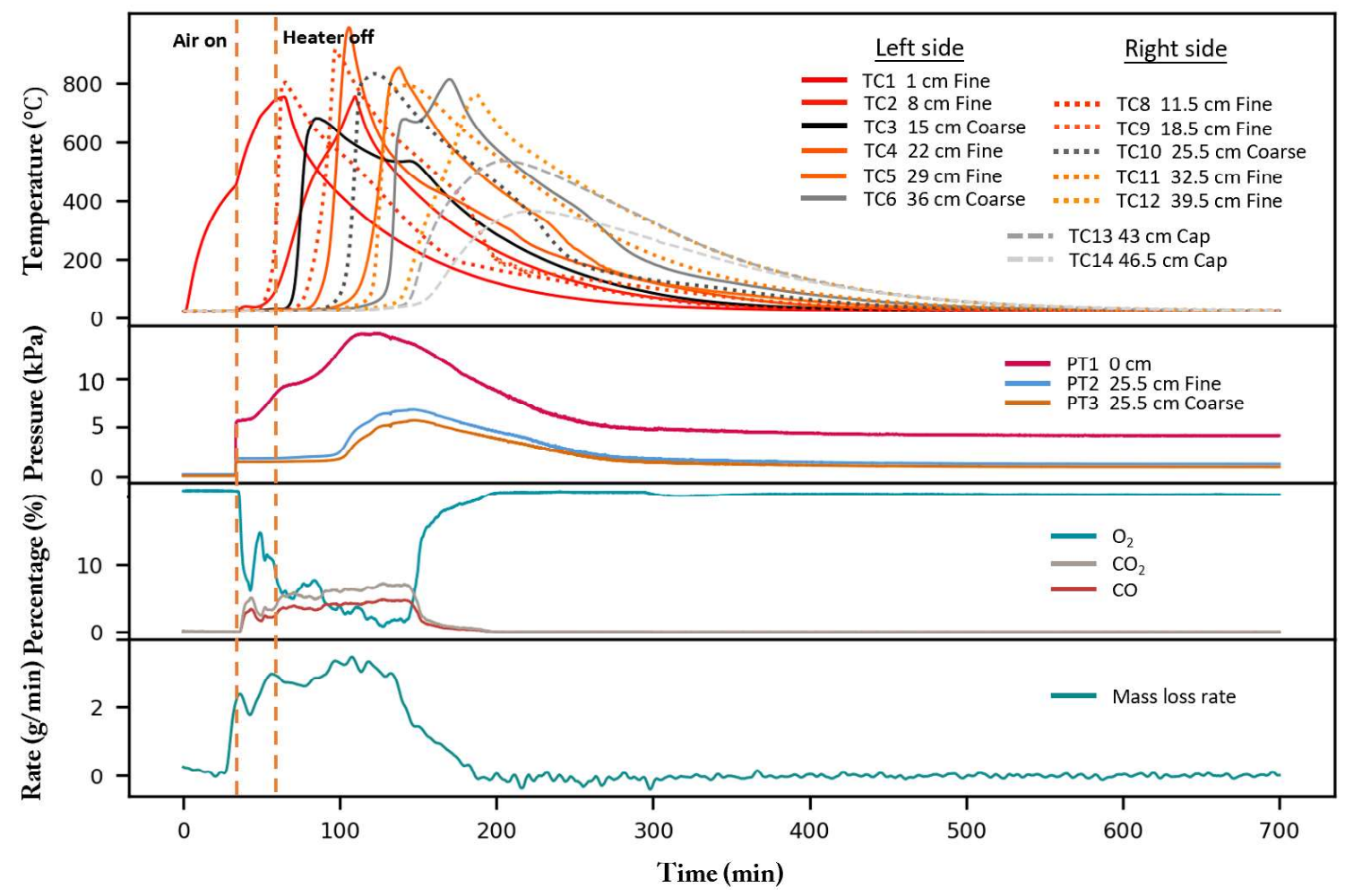

Figure 3.11: Temperature, pressure, emission and mass loss rate profiles for Complex: Disconnected Coarse.

The comparison of Parallel 1 and Complex: Disconnected Coarse reveals that the increase of a certain pattern of the heterogeneity complexity may help evenly distribute air amongst the layers in the system. The mix of the coarse and fine layers and disconnected coarse layers resulted in no significant air bypassing of fine layers; this observation was supported by the higher observed pressure drop (14.5 $\mathrm{kPa}$ here versus $3.2 \mathrm{kPa}$ in Parallel 1). In addition, the series and parallel distribution of coarse layers led to a higher proportion of air traversing horizontally through the middle of the column and thus crossing the fine layers. Both factors likely increased the air flux in the fine layers. Indeed, remaining GAC on excavation was only found in minor amounts adjacent to the wall at the edge of the fine layers, supporting the hypothesis that air flux was sufficient to sustain the smouldering propagation in the bulk of the fine layers (see Appendix C). 


\subsubsection{Simulation of Parallel 1-3 and two Complex cases}

ISSM simulations were set up for Parallel 1-3 and Complex: Disconnected Coarse to better understand experimental results and the effect of heterogeneity on the smouldering reaction, which has not been calibrated for any parameters in this study. Figure 3.12 shows the distribution of local air flux vectors and pressures in these simulations. Air flux vectors in the model can change with time as the smouldering reaction propagates and eliminates fuel (Solinger et al., 2019). However, in these cases, since the GAC concentration was small, the air flux maps did not change significantly with time. The average air flux estimated from the model for fine and coarse layers respectively: 0.11 and $4.89 \mathrm{~cm} / \mathrm{s}$ in Parallel 1, 0.51 and $4.49 \mathrm{~cm} / \mathrm{s}$ in Parallel 2, 1.43 and $3.57 \mathrm{~cm} / \mathrm{s}$ in Parallel 3, were similar to the experimental calculations presented above. While experimental calculations were not possible for Complex: Disconnected Coarse (insufficient number of pressure measurements), the ISSM predicted air flux in the fine layers to be faster than in Parallel $1(1.5 \mathrm{~cm} / \mathrm{s}$ versus $0.11 \mathrm{~cm} / \mathrm{s})$ even though they both had 1.5 orders of magnitude permeability difference; this supports the differences in smouldering behaviour described for the experiment. The pressure changes predicted in these simulations are discussed in Appendix F. 

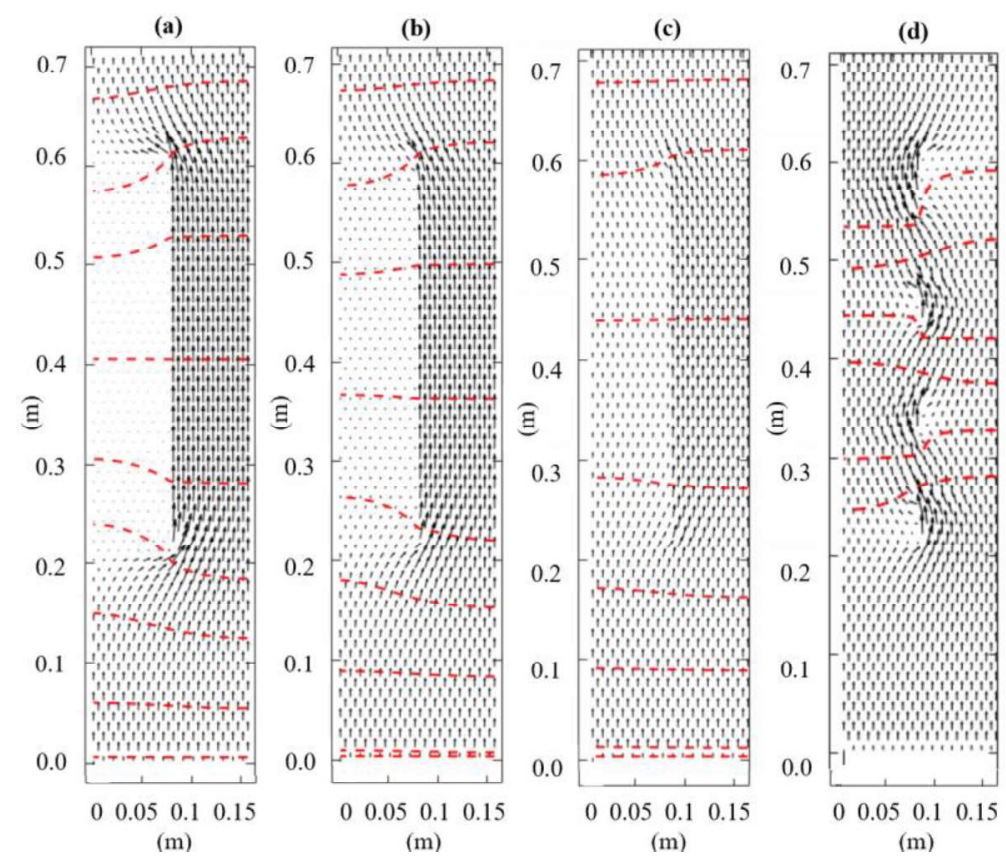

Figure 3.12: Air and pressure distributions (red dash-line) in simulations: (a) Parallel 1, (b) Parallel 2, (c) Parallel 3 and (d) Complex: Disconnected Coarse. Each arrow is a velocity vector, with its magnitude expressed by length and direction expressed by orientation. Red dash lines represent pressure contours.

Figure 3.13 illustrates the final distribution of clean and contaminated material after smouldering was complete for Parallel 1-3 and Complex: Disconnected Coarse. These reveal the extent to which smouldering was predicted to propagate through parallel layers. Note that the time-lapse images for all four simulations are presented in Appendix G. In all four cases, self-sustained smouldering was predicted to completely treat the coarse layer, which matched experimental observations. In Parallel 1, no smouldering was predicted in the fine layer due to air flux below the threshold required for smouldering, matching experimental observations. In Parallel 2, the model correctly predicted the ignition and then rapid quenching of smouldering due to the limited air flux. In Complex: Disconnected Coarse, the model correctly predicted almost complete treatment of all layers due to the enhanced air flux in all fine layers. However, in Parallel 3, the fine layer was predicted to be completely treated, which was different from the 
experimental results where smouldering propagated upwards in the fine layer until heat transfer to the clean coarse layer led to its extinction. The main reason for this discrepancy was that heat transfer was neglected by this model (MacPhee et al., 2012), and thus quenching of a reaction due only to excessive heat losses cannot be simulated.
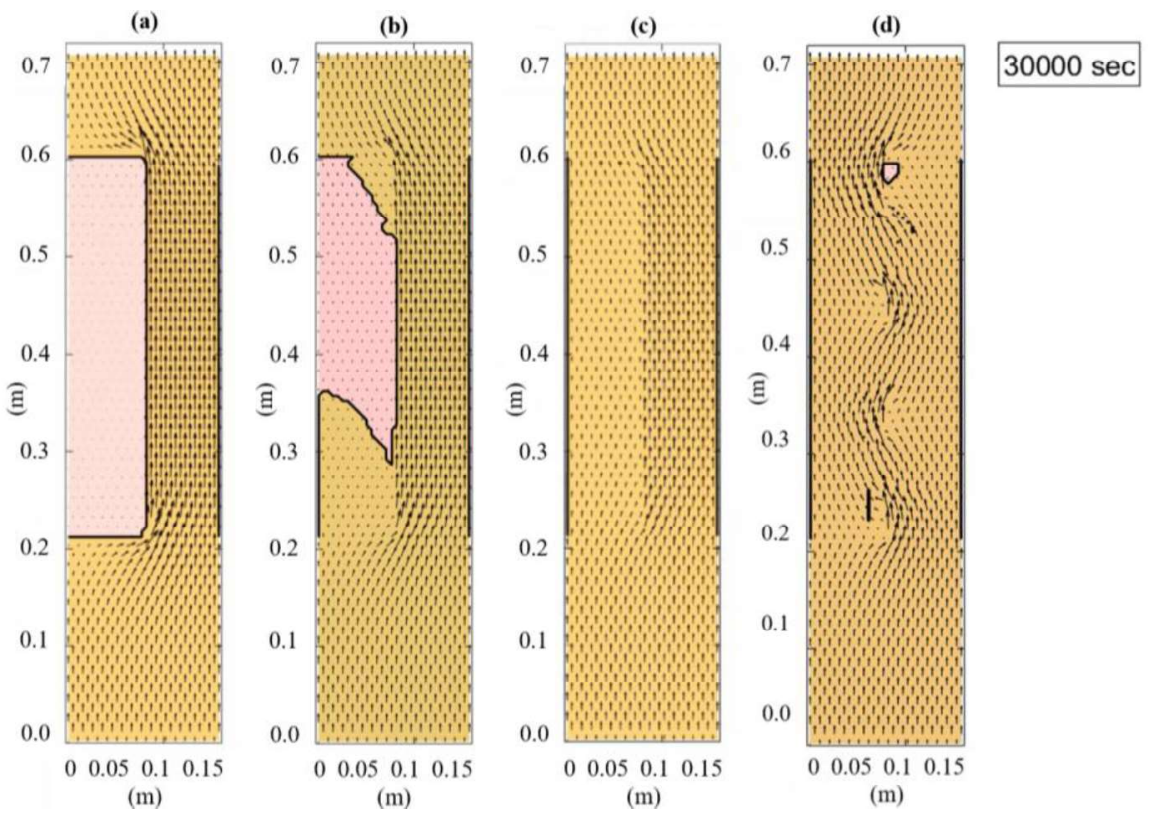

Figure 3.13: Final status of smouldering reaction in four simulations: (a) Parallel 1, (b) Parallel 2, (c) Parallel 3 and (d) Complex: Disconnected Coarse. Yellow represents clean sand while pink represents contamination left behind in the treatment zone.

Figure 3.14 shows the comparison of air distribution and smouldering development in two Complex cases. Different from the complete treatment of fine layers in Complex: Disconnected Coarse, connected coarse layers created a preferential pathway from the bottom to the top and led to the airflow channelling around the fine layers, causing smouldering bypassing, and resulting in the bulk of the fine layers being untreated in Complex: Connected Coarse. Air flux in the fine layer was estimated to be less than in the Complex: Disconnected Coarse $(0.35 \mathrm{~cm} / \mathrm{s}$, versus $1.5 \mathrm{~cm} / \mathrm{s})$. Due to limited air flux $(<0.5 \mathrm{~cm} / \mathrm{s})$, ISSM predicted the smouldering failure in the fine layer in this case, similar 
to the simulation result of smouldering through low permeability lenses in previous research (MacPhee et al., 2012). The difference between two Complex cases reveals the importance of the pattern of heterogeneity on the air distribution and successful smouldering.
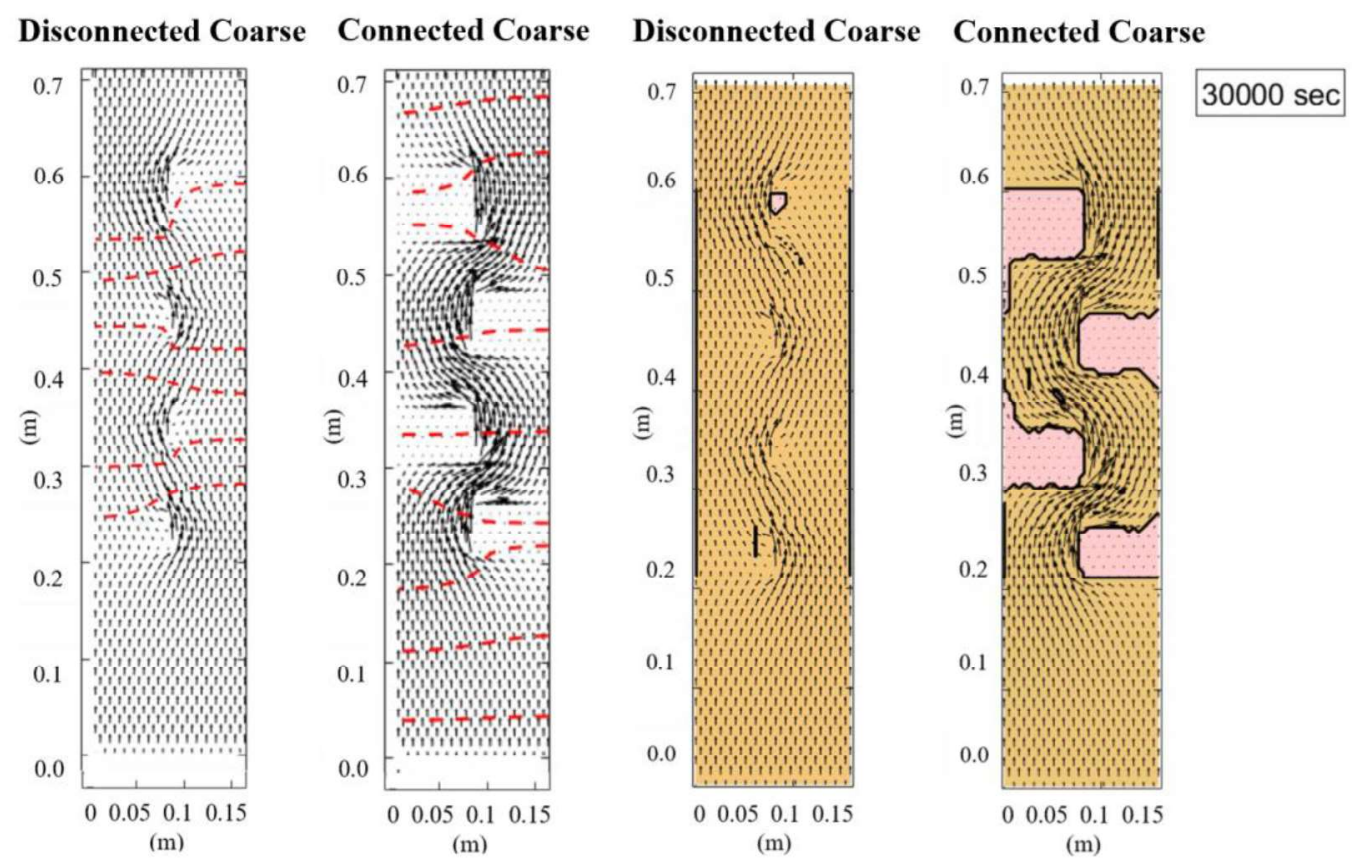

Figure 3.14: Comparison of the simulation between two Complex cases: Disconnected Coarse and Connected Coarse scenarios.

\subsection{Summary and Conclusions}

For the first time, self-sustained smouldering propagation in systems with heterogeneous permeability has been examined experimentally. Smouldering across layers in series was successful where the permeability contrast was up to 1000 -fold (from $1.3 \times 10^{-9} \mathrm{~m}^{2}$ to $1.1 \times 10^{-12} \mathrm{~m}^{2}$ ). The reaction was relatively unchanged across the boundary, with consistent peak temperature and smouldering velocity reducing slightly with decreased permeability. Smouldering tended to propagate in the direction of airflow, due to the importance of both oxygen to the oxidation reaction and forward convective heat transfer 
to energy recycling. Thus, when air flux was continuous between adjacent layers, smouldering was relatively unaffected.

Experiments and modelling of layers in parallel reveal that the distribution of the airflow between adjacent layers was determined by the permeability ratio between them, where the absolute permeability of each layer is of minor significance under the fixed injection rate condition. When the permeability ratio caused the air flux in one layer to fall below the threshold for self-sustained smouldering in laboratory experiments $(0.5 \mathrm{~cm} / \mathrm{s})$, the reaction terminated. When air flux exceeded this threshold in both layers, smouldering was observed in both layers. However, the relative air fluxes still mattered, as thermal coupling had negative impacts in cases where adjacent layers exhibited reactions that propagated at different rates. For the layer with slower propagation, extinction was induced due to heat losses to the adjacent layer that was cooling after treatment. For the layer with faster propagation, the reaction velocity was reduced due to heat transfer to the untreated adjacent layer; however, this did not lead to extinction in the coarse layer in any of cases examined. In addition to permeability ratio, the complexity of heterogeneity (i.e., lengths and distribution of lenses, disconnection of coarse lenses) was found to play an important role. Complex distributions can generate the benefits of layers in series to the extent that they offset the negative consequences of layers in parallel. Successful smouldering of all layers occurred in scenarios where a continuous, high permeability path did not exist, and therefore (i) air could not largely bypass low permeability zones, and (ii) smouldering propagated at relatively similar rates in all pathways. Clearly, the magnitude of heterogeneity of materials and their distribution are of prime importance, as they dictate the air distribution flow field that underlies the ignition of smouldering reactions and their 
relative propagation rates, which in turn dictate the local energy balances upon which selfsustained smouldering depends. Moreover, it is clear that hand calculations, for simple cases, and straightforward flow modelling, for more complex cases, can predict the expected airflow patterns for given scenarios and those can be used to make informed estimates of smouldering behaviour.

This work suggests that smouldering should be successful in numerous heterogeneous scenarios in the field. However, one scenario that may be challenging is treating a contaminated fine layer when a parallel coarse layer exists that is extensive in the direction of airflow. One way this may be minimized for in situ smouldering remediation is to ensure that smouldering is carried out in each layer separately, i.e., air injection well screens are entirely within a single layer, thereby preventing dividing the injected air between multiple layers. High resolution site characterization combined with treating individual layers sequentially has already been demonstrated in situ (Scholes et al., 2015).

It is acknowledged that the experimental set-up here only included simple layers in series, parallel and a checkerboard, which illustrated the principles at work but did not adequately reproduce the complexity of heterogeneity expected in real subsurface scenarios. Modelling would be the most appropriate approach to explore such systems. In addition, these results used column experiments with thin layers, which exhibit high heat losses relative to larger (field) systems. This means that the extinction events observed here are highly conservative, and smouldering reactions in field scale systems may be more robust (e.g., self-sustained at lower air fluxes, less sensitive to lateral heat losses). Moreover, this study used granular activated carbon whereas NAPL may exhibit a greater 
impact on intrinsic permeability at typical saturations. In addition, contrasting lenses

exhibiting similar saturations of fuel may be not common in the field, where low

permeability zones are less likely to have high concentration of contaminants, which may

also affect the smouldering performance. Nevertheless, it is expected that these

limitations do not alter the main conclusions of the work.

\subsection{References}

Akkutlu, I. Y., \& Yortsos, Y. C. (2005). The Effect of Heterogeneity on In-Situ Combustion: Propagation of Combustion Fronts in Layered Porous Media. SPE Journal, 10(4), 394-404.

Carter, K. E., \& Farrell, J. (2010). Removal of Perfluorooctane and Perfluorobutane Sulfonate from Water via Carbon Adsorption and Ion Exchange. Separation Science and Technology, 45(6), 762-767.

Dosanjh, S. S., Pagni, P. J., \& Fernandez-Pello, A. C. (1987). Forced cocurrent smoldering combustion. Combustion and Flame, 68(2), 131-142.

Drysdale, D. (2011). Spontaneous Ignition within Solids and Smoldering Combustion An introduction to fire dynamics. John Wiley \& Sons, Ltd. 512 p.

Gerhard, J. I., \& Kueper, B. H. (2003a). Capillary pressure characteristics necessary for simulating DNAPL infiltration, redistribution, and immobilization in saturated porous media. Water Resources Research, 39(8).

Gerhard, J. I., \& Kueper, B. H. (2003b). Relative permeability characteristics necessary for simulating DNAPL infiltration, redistribution, and immobilization in saturated porous media. Water Resources Research, 39(8).

Gerhard, J. I., \& Kueper, B. H. (2003c). Influence of constitutive model parameters on the predicted migration of DNAPL in heterogeneous porous media. Water Resources Research, 39(10).

Gerhard, J. I., Gavin, G. P., \& Torero, J. L. (2020). Star: a uniquely sustainable in situ and ex situ remediation process. Sustainable Remediation of Contaminated Soil and Groundwater. Butterworth-Heinemann. 221-246

Gill, R., Harbottle, M., Smith, J., \& Thornton, S. (2014). Electrokinetic-enhanced bioremediation of organic contaminants: A review of processes and environmental applications. Chemosphere, 107, 31-42.

Gorokhovski, V. (2012). Effective Parameters of Hydrogeological Models. Berlin: Springer. $168 \mathrm{p}$. 
Grant, G. P., Major, D., Scholes, G. C., Horst, J., Hill, S., Klemmer, M. R., \& Couch, J. N. (2016). Smoldering combustion (STAR) for the treatment of contaminated soils: Examining limitations and defining success. Remediation Journal, 26(3), 27-51.

Hasan, T., Gerhard, J. I., Hadden, R., \& Rein, G. (2015). Self-sustaining smouldering combustion of coal tar for the remediation of contaminated sand: Twodimensional experiments and computational simulations. Fuel, 150, 288-297.

Kinsman, L., Torero, J., \& Gerhard, J. (2017). Organic liquid mobility induced by smoldering remediation. Journal of Hazardous Materials, 325, 101-112.

Kueper, B. H., Stroo, H.F., Vogel, C. M., \& Ward, C. H. (2014). Chlorinated Solvent Source Zone Remediation. New York: Springer. 713 p.

Leonards, G. A. (1962). Engineering Properties of soils. Foundation Engineering McGraw-Hill, New York. 66-240

Liu, C. J., Wernerb, D., \& Bellona, C. (2019). Removal of per- and polyfluoroalkyl substances (PFASs) from contaminated groundwater using granular activated carbon: a pilot-scale study with breakthrough modeling. Environmental Science: Water Research \& Technology, 5, 1844-1853.

MacPhee, S. L., Gerhard, J. I., \& Rein, G. (2012). A novel method for simulating smoldering propagation and its application to STAR (Self-sustaining Treatment for Active Remediation). Environmental Modelling \& Software, 31, 84-98.

Major, D. W. (2019). Demonstration of Smoldering Combustion Treatment of PFASimpacted Investigation-Derived Waste (SERDP Project ER18-1593). Canada. Geosyntec Consultants, Inc.

NRC (National Research Council). (2013). Alternatives for managing the nation's complex contaminated groundwater sites. Washington, DC: The National Academies Press. 422 p.

Ohlemiller, T. J. (1985). Modeling of smoldering combustion propagation. Progress in energy and combustion science, 11(4), 277-310.

Pironi, P., Switzer, C., Rein, G., Fuentes, A., Gerhard, J. I., \& Torero, J. L. (2009). Smallscale forward smouldering experiments for remediation of coal tar in inert media. Proceedings of the Combustion Institute, 32(2), 1957-1964.

Pironi, P., Switzer, C., Gerhard, J. I., Rein, G., \& Torero, J. L. (2011). Self-sustaining smoldering combustion for NAPL remediation: laboratory evaluation of process sensitivity to key parameters. Environmental Science \& Technology, 45(7), 29802986.

Rashwan, T. L., Gerhard, J. I., \& Grant, G. P. (2016). Application of self-sustaining smoldering combustion for the destruction of wastewater biosolids. Waste Management, 50, 201-212. 
Reddy, K. R., \& Adams, J. A. (2001). Effects of soil heterogeneity on airflow patterns and hydrocarbon removal during in situ air sparging. Journal of geotechnical and geoenvironmental engineering, 127(3), 234-247.

Richards, G. D. (1990). An elliptical growth model of forest fire fronts and its numerical solution. International Journal for Numerical Methods in Engineering, 30(6), 1163-1179.

Richards, G.D. (1995). "A General Mathematical Framework for Modeling TwoDimensional Wildland Fire Spread." International Journal of Wildland Fire, 5(2), 63-72.

Rosenbaum, W. A. (2019). Environmental politics and policy. Washington, D.C: CQ Press, a Division of Sage. 352 p.

Salman, M., Gerhard, J. I., Major, D. W., Pironi, P., \& Hadden, R. (2015). Remediation of trichloroethylene-contaminated soils by star technology using vegetable oil smoldering. Journal of Hazardous Materials, 285, 346-355.

Switzer, C., Pironi, P., Gerhard, J., Rein, G., \& Torero, J. (2009). Self-sustaining smoldering combustion: a novel remediation process for non-aqueous-phase liquids in porous media. Environmental Science \& Technology, 43(15), 58715877.

Switzer, C., Pironi, P., Gerhard, J. I., Rein, G., \& Torero, J. L. (2014). Volumetric scaleup of smouldering remediation of contaminated materials. Journal of Hazardous Materials, 268, 51-60.

Scholes, G.C., Gerhard, J.I., Grant, G.P., Major, D.W., Vidumsky, J.E., Switzer, C., \& Torero, J.L. (2015). Smoldering remediation of coal-tar-contaminated soil: pilot field tests of STAR. Environmental Science \& Technology. 49, 14334-14342.

Selvadurai, P. A., \& Selvadurai, A. P. S. (2014). On the effective permeability of a heterogeneous porous medium: the role of the geometric mean. Philosophical Magazine, 94(20), 2318-2338.

Seol, Y., Zhang, H., \& Schwartz, F. W. (2003). A Review of In Situ Chemical Oxidation and Heterogeneity. Environmental and Engineering Geoscience, 9(1), 37-49.

Solinger, R., Grant, G.P., Scholes, G.C., Murray, C., \& Gerhard, J.I. (2019). STARx hottpad for smouldering treatment of waste oil sludge: proof of concept and sensitivity to key design parameters. Accepted for publication in Waste Management \& Research.

Torero, J., \& Fernandez-Pello, A. (1996). Forward smolder of polyurethane foam in a forced air flow. Combustion and Flame, 106(1-2), 89-109.

U.S. EPA (U.S. Environmental Protection Agency). (2002). Groundwater remedies selected at superfund sites. EPA-542-R-01-022. Office of Solid Waste and Emergency Response. 38 p. 
U.S. EPA (U.S. Environmental Protection Agency). (2003). The DNAPL Remediation Challenge: Is There a Case for Source Depletion? EPA-600-R-03-143. Office of Research and Development. 129 p.

Yermán, L., Hadden, R. M., Carrascal, J., Fabris, I., Cormier, D., Torero, J. L., Gerhard, J. I., Krajcovic, M., Pironi, P., \& Cheng, Y.-L. (2015). Smouldering combustion as a treatment technology for faeces: Exploring the parameter space. Fuel, 147, 108-116.

Yermán, L., Wall, L., Torero, J., Gerhard, J. I., \& Cheng, Y. -L. (2016). Smoldering combustion as a treatment technology for feces: sensitivity to key parameters. Combustion Science and Technology, 188(6), 968-981.

Zanoni, M. A., Torero, J. L., \& Gerhard, J. I. (2017). Determination of the interfacial heat transfer coefficient between forced air and sand at Reynold's numbers relevant to smouldering combustion. International Journal of Heat and Mass Transfer, 114, 90-104.

Zanoni, M. A., Torero, J. L., \& Gerhard, J. I. (2019). Delineating and explaining the limits of self-sustained smouldering combustion. Combustion and Flame, 201, 7892. 


\section{Chapter 4}

\section{Conclusions and Recommendations}

\subsection{Conclusions}

This thesis explored the effect of intrinsic permeability and heterogeneity on smouldering propagation. A series of laboratory smouldering experiments using granular activated carbon (GAC) as the model fuel, mixed with various sands were used to understand the effects of permeability heterogeneity in various scenarios. The GAC and sand layers with varying permeability values were arranged in series, in parallel and in a checkerboard relative to the direction of airflow and reaction propagation. These experiments were quantified in terms of temperature profiles, reaction velocities, pressure changes, and in situ air fluxes in order to assess the smouldering robustness under different situations. In addition, numerical modelling (ISSM) was used to simulate Parallel 1-3 and Complex cases, which accounted for the impact of permeability heterogeneity on air velocities, providing additional insights into better understanding and interpreting the experimental results.

The main findings are summarized:

- The experiments with varying permeability layers in Series showed that smouldering propagated successfully through all layers, even with the permeability contrast up to 1000 -fold (from $1.3 \times 10^{-9} \mathrm{~m}^{2}$ to $1.1 \times 10^{-12} \mathrm{~m}^{2}$ ).

- The experiments with varying permeability layers in Parallel showed that smouldering propagated successfully in coarse layers, whereas failed in the fine layers. 
- Sufficient air flux was vital for self-sustained smouldering; both experimental results and model simulations showed that smouldering failed when local air fluxes through a layer was less than $0.5 \mathrm{~cm} / \mathrm{s}$, which occurred when high permeability difference between parallel layers diverted the majority of flow through the coarse layer.

- The experiments with varying permeability layers in a complex pattern (i.e., disconnected coarse layers in the mixture) showed that the lengths and distribution of low permeability lenses dictated the distribution of air and thus played an important role in smouldering propagation. The effect of air channelling through high permeable pathways was dampened as the low permeability lenses were staggered and coarse lenses were disconnected throughout the column, resulting in more uniform air flux distribution and successful smouldering propagation.

- Heat conduction and loss was another important factor, as a smouldering reaction with sufficient air flux might still quench with high heat losses. In Parallel 3, extinction in the low permeability layer was likely caused by lateral conductive heat transfer, or thermal coupling, from smouldering layer (low permeability) to treated layer (high permeability). In addition, thermal coupling affected the reaction in the coarse layer, as the smouldering reaction was slowed when heat was lost to an adjacent layer that was not smouldering.

- The ISSM results agreed with expectations as the low air fluxes simulated in the low permeability layer should drive extinction. However, the effects of the temperature (e.g., heat losses or changing air properties) were not simulated, 
which helped explain the discrepancies between model results and experimental observations in some cases.

- Considering the practical implications of this work, smouldering should be successful in numerous heterogeneous scenarios; however, high permeability layers that are long in the direction of smouldering may lead to air channelling and smouldering failure in adjacent low permeability layers if attempts are made to treat both at once with a well screened over both layers.

\subsection{Recommendations}

As this study serves as an initial investigation in exploring the effect of intrinsic permeability and heterogeneity on smouldering propagation, there are some improvements and recommendations for future work.

The following is recommended:

- Additional parallel tests with low contrasting permeability ratio may be useful to identify a critical ratio, where smouldering in the fine layer can be self-sustained regardless of the smouldering condition in the parallel coarse layer. Besides, it would also be beneficial to explore the relationship between permeability ratio, air flux and reaction velocity in each layer to better understand the effect of thermal coupling on heterogeneity.

- Since the experimental set-up here only considered simple layers in series and in parallel, which did not completely reproduce the complexity of real subsurface heterogeneity, additional experiments may be valuable, e.g., 3-D smouldering into 
disconnected lenses. Modelling would be the most appropriate approach to explore these cases.

- Since only solid GAC was studied in heterogeneous smouldering tests, this may not completely reproduce the actual smouldering performance in the field site, which typically contains liquid hydrocarbons that may flow, especially when heated and their viscosity decreases, which may also have high saturations, thereby also changing the air permeability. Therefore, a similar study with liquid fuels may be valuable, where fuel mobility should be well characterized.

- The effect of scale needs to be investigated. As studied in previous research, the quenching limits are influenced by the system scale (Switzer et al., 2014). Increasing the scale may reduce the heat losses and increase the robustness of the fine layer reaction in layers in parallel tests.

- Finally, a numerical model that incorporates heat transfer effects and handles permeability contrasts in multiple dimensions should be developed and then applied to smouldering simulations in order to provide further insight.

\subsection{Reference}

Switzer, C., Pironi, P., Gerhard, J. I., Rein, G., \& Torero, J. I. (2014). Volumetric scaleup of smoldering remediation of contaminated materials. Journal of Hazardous Materials, 268(15), 51-60. 


\section{Appendices}

\section{Appendix A: Supporting Information for Sand Properties}

Particle size distributions of three purchased silica sands (\#12, \#505 and \#106) are summarized in Figure A-1, in which the results of silica sand \#12 and \#505 are from Bell \& MacKenzie Co. Ltd, and the data for silica sand \#106 is from the combination of hydrometer analysis and sieve analysis (140 mesh and $200 \mathrm{mesh})$.

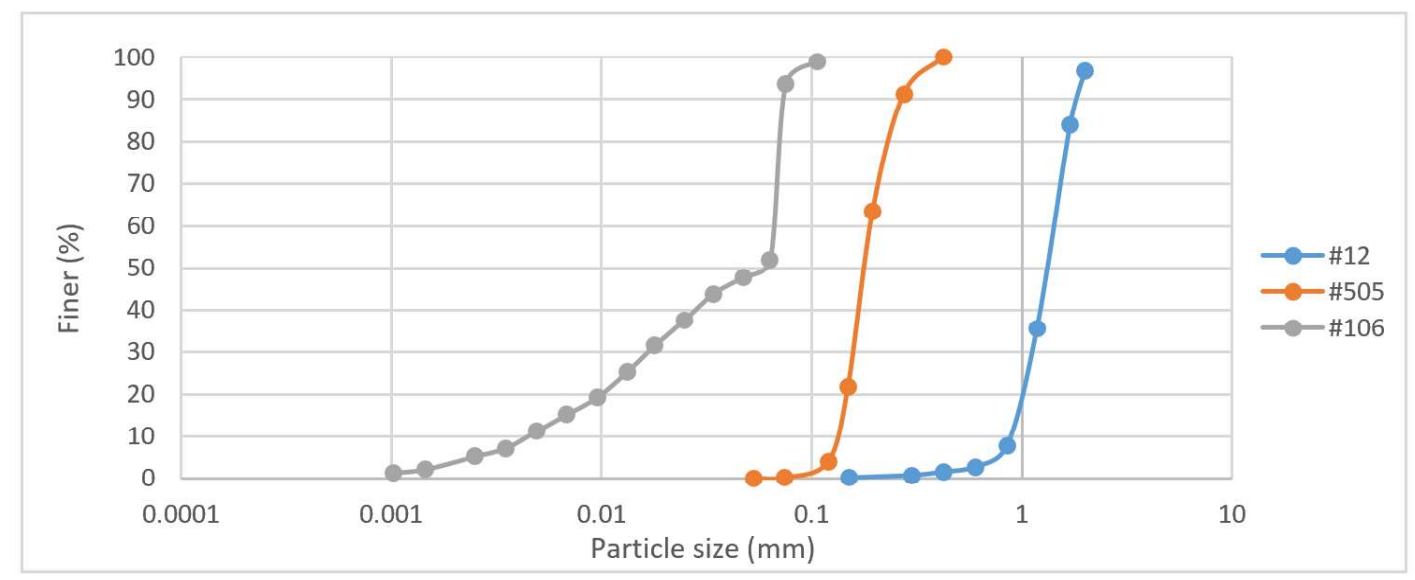

Figure A-1: Particle size distribution curve for silica sand \#12, \#505 and \#106.

The permeability and porosity of 33 sand mixtures with different constituents are summarized in Table A-1, in which permeability values were measured in a permeability cell following ASTM D6539-00. 
Table A-1: Permeability and Porosity of Different Porous Media

\begin{tabular}{|c|c|c|c|c|c|c|}
\hline Sand constituents & No. & Sieved Coarse $(1.18-2.00 \mathrm{~mm})$ & Sieved Fine $(0.125-0.250 \mathrm{~mm})$ & Powder $(\bar{d}=0.043 \mathrm{~mm})$ & Permeability $\left(\mathrm{m}^{2}\right)$ & Porosity (\%) \\
\hline \multirow{8}{*}{ Coarse + Fine } & 1 & $100.0 \%$ & $0.0 \%$ & क...... & $1.3 \times 10^{-9}$ & $44.8 \%$ \\
\hline & 2 & $90.0 \%$ & $10.0 \%$ & -......- & $7.1 \times 10^{-10}$ & $36.4 \%$ \\
\hline & 4 & $70.0 \%$ & $30.0 \%$ & ........ & $2.7 \times 10^{-10}$ & $33.2 \%$ \\
\hline & 5 & $60.0 \%$ & $40.0 \%$ & ....... & $1.2 \times 10^{-10}$ & $31.4 \%$ \\
\hline & 6 & $50.0 \%$ & $50.0 \%$ & -....... & $3.9 \times 10^{-11}$ & $30.1 \%$ \\
\hline & 9 & $20.0 \%$ & $80.0 \%$ & ........ & $2.7 \times 10^{-11}$ & $35.3 \%$ \\
\hline & 10 & $10.0 \%$ & $90.0 \%$ & -...... & $2.4 \times 10^{-11}$ & $36.5 \%$ \\
\hline & 11 & $0.0 \%$ & $100.0 \%$ & ……- & $1.9 \times 10^{-11}$ & $45.4 \%$ \\
\hline \multirow{8}{*}{ Fine + Powder } & 12 & (n) & $100.0 \%$ & $0.0 \%$ & $1.9 \times 10^{-11}$ & $45.4 \%$ \\
\hline & 13 & ....... & $95.0 \%$ & $5.0 \%$ & $6.6 \times 10^{-12}$ & $37.9 \%$ \\
\hline & 14 & ....... & $90.0 \%$ & $10.0 \%$ & $2.5 \times 10^{-12}$ & $34.7 \%$ \\
\hline & 19 & …… & $65.0 \%$ & $35.0 \%$ & $1.3 \times 10^{-13}$ & $30.0 \%$ \\
\hline & 20 & …… & $60.0 \%$ & $40.0 \%$ & $1.2 \times 10^{-13}$ & $28.9 \%$ \\
\hline & 21 & …… & $55.0 \%$ & $45.0 \%$ & $9.8 \times 10^{-14}$ & $30.0 \%$ \\
\hline & 22 & (........ & $50.0 \%$ & $50.0 \%$ & $7.4 \times 10^{-14}$ & $30.0 \%$ \\
\hline & 23 & $\ldots$ & $0.0 \%$ & $100.0 \%$ & $5.1 \times 10^{-14}$ & $39.7 \%$ \\
\hline \multirow{6}{*}{ Coarse + Fine + Powder } & 24 & $50.0 \%$ & $45.0 \%$ & $5.0 \%$ & $9.7 \times 10^{-12}$ & $27.6 \%$ \\
\hline & 25 & $47.5 \%$ & $47.5 \%$ & $5.0 \%$ & $6.7 \times 10^{-12}$ & $27.2 \%$ \\
\hline & 26 & $60.0 \%$ & $30.0 \%$ & $10.0 \%$ & $2.2 \times 10^{-12}$ & $25.9 \%$ \\
\hline & 27 & $55.0 \%$ & $35.0 \%$ & $10.0 \%$ & $2.2 \times 10^{-12}$ & $23.1 \%$ \\
\hline & 28 & $50.0 \%$ & $40.0 \%$ & $10.0 \%$ & $1.9 \times 10^{-12}$ & $25.6 \%$ \\
\hline & 29 & $45.0 \%$ & $45.0 \%$ & $10.0 \%$ & $2.0 \times 10^{-12}$ & $27.1 \%$ \\
\hline
\end{tabular}

The repeated permeability tests from different batches of sand \#33 were carried out based on ASTM D6539-00 (Table A-2), in which low Relative Standard Deviation (RSD) values reveal that the applied mixing procedure during the experiments produced homogeneous sand mixture.

Table A-2: Repeatable Permeability Test for Sand \#33

\begin{tabular}{ccc}
\hline Tests & Permeability(m $\left.\mathbf{m}^{2}\right)$ & Porosity(\%) \\
\hline 1 & $1.9 \times 10^{-13}$ & 22.5 \\
2 & $1.6 \times 10^{-13}$ & 21.7 \\
3 & $2.2 \times 10^{-13}$ & 23.7 \\
4 & $1.5 \times 10^{-13}$ & 21.9 \\
5 & $1.9 \times 10^{-13}$ & 23.1 \\
6 & $2.2 \times 10^{-13}$ & 24 \\
\hline Standard Deviation & $2.7 \times 10^{-14}$ & 0.86 \\
Mean & $1.9 \times 10^{-13}$ & 23 \\
Relative Standard Deviation (RSD) & $14.2 \%$ & $3.8 \%$ \\
\hline
\end{tabular}

Mixing various weight fractions of the sieved coarse and fine sands with the silica powder allowed the production of a wide range of controlled intrinsic permeability values. Based 
on Table A-1, Figures A-2, A-3 show changes of permeability and porosity of porous media as the mass fraction of fine materials in coarse materials (i.e., sieved fine into sieved coarse, powder into sieved fine).

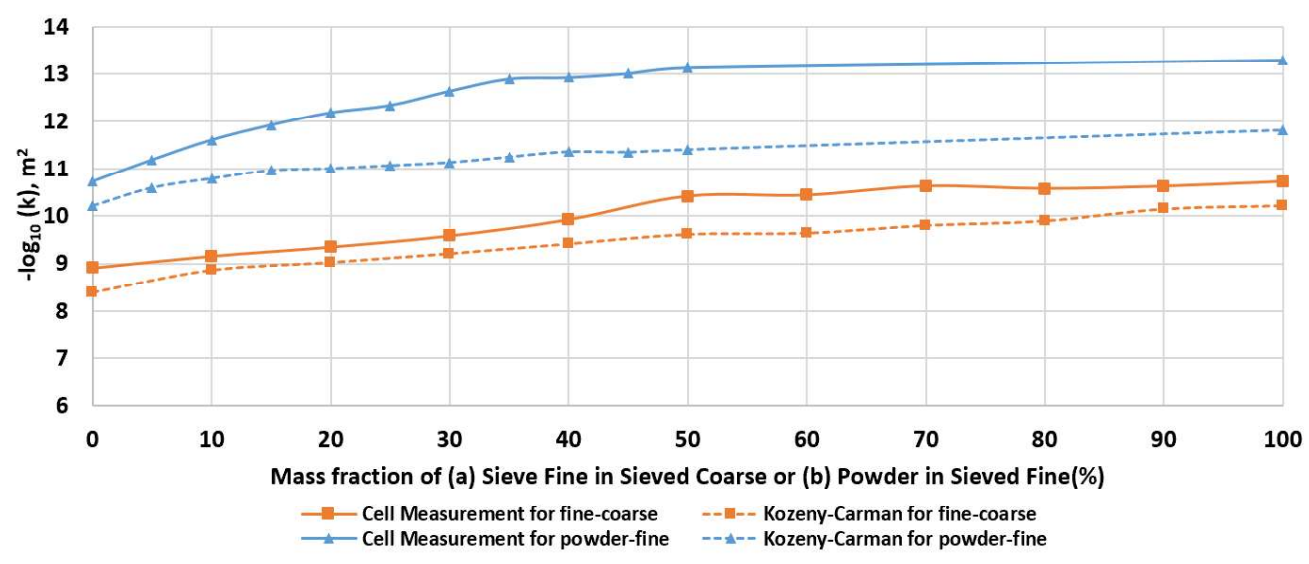

Figure A-2: Permeability changes as the mass fraction of fine materials.

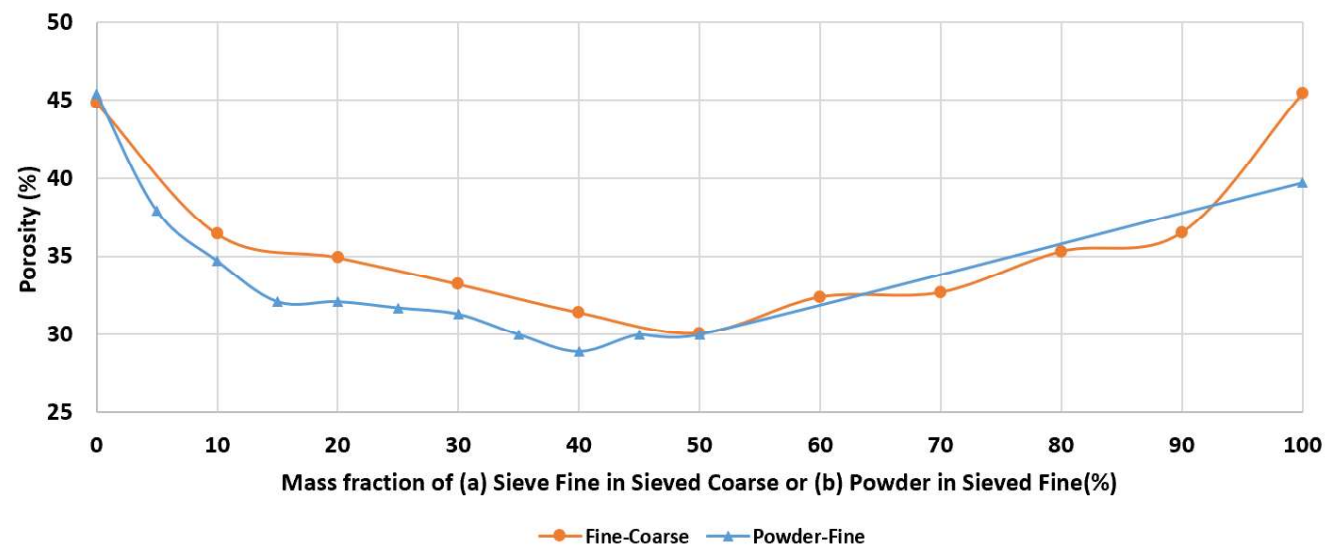

Figure A-3: Porosity changes as the mass fraction of fine materials.

With respect to the permeability of sand mixture, it was dominated by the lowest permeability material inside, especially when the fraction of finer material exceeded one certain value, the permeability of mixture was close to the intrinsic permeability of the pure fine material. Permeability values calculated from the Kozeny-Carman equation (Equation 1) were plotted in the same graph with experimental data (Figure A-2). It shows a similar 
trend of permeability changes as experimental results, indicating that the permeability was affected by not only the average particle size but also the porosity of the mixture.

$$
k=\frac{\emptyset^{3}}{36 \kappa(1-\emptyset)^{2}} d^{2}
$$

where $\kappa$ is the $\mathrm{KC}$ constant, which applied 5 here; $d(\mathrm{~m})$ is the mean diameter of hypothetical spherical solid particles with the same specific surface area.

However, the permeability value from Kozeny-Carman equation was lower than the measurement value, this may be due to the underestimate of $\mathrm{KC}$ constant here. $\mathrm{KC}$ constant is an empirical value which is affected by porosity, microstructure of pores and capillaries.

As for the porosity of the mixture, Figure A-3 shows that the porosity was not significantly affected by the size of sand particles either in the coarse mixture or fine mixture, the porosity of the mixture was low when sands were poorly sorted whereas it was high in well-sorted sands. 


\section{Appendix B: Repeatability of Smouldering Reaction}

Figure B-1 shows the experimental set-ups for three scenarios: Column A with Upward Smouldering, Column B with Upward Smouldering and Column B with Downward Smouldering. Table B-1 shows the similar behaviours of three identical smouldering tests in Column A, and Column B with two opposite smouldering directions with the same GAC saturation (20 g GAC/kg Sand), same air flux $(5 \mathrm{~cm} / \mathrm{s})$. The uncertainties of propagation velocity and average peak temperature in three tests were overlapping, revealing that there was no obvious difference of smouldering performance between different columns and different smouldering directions.

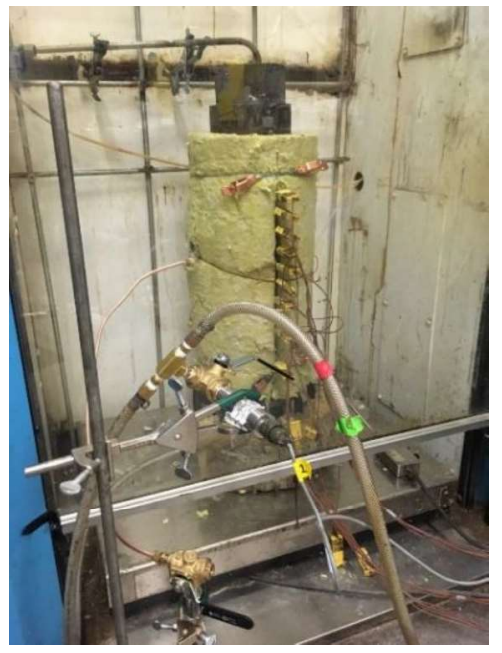

(a)

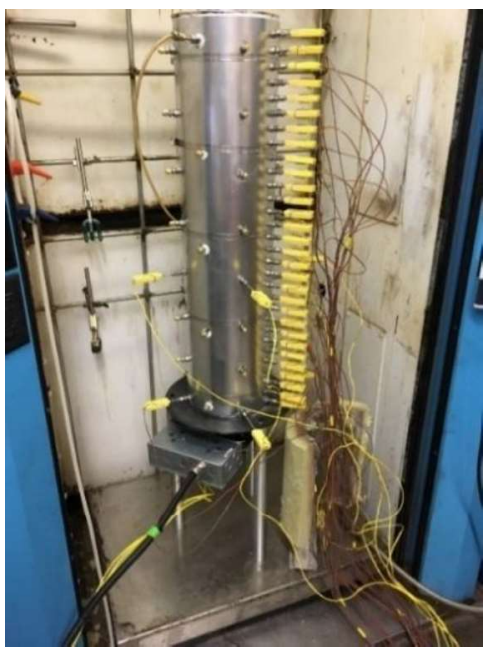

(b)

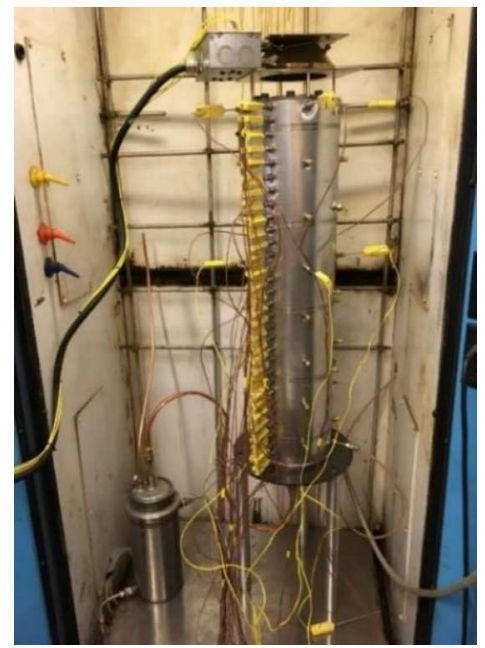

(c)

Figure B-1: Experiments of (a) Column A with Upward Smouldering, (b) Column B with Upward Smouldering and (c) Column B with Downward Smouldering. 
Table B-1: Comparison of Upward and Downward Smouldering

\begin{tabular}{cccc}
\hline Column & $\begin{array}{c}\text { Smouldering } \\
\text { Direction }\end{array}$ & $\begin{array}{c}\text { Average Front } \\
\text { Velocity }(\mathbf{m m} / \mathbf{m i n})\end{array}$ & $\begin{array}{c}\text { Average Peak } \\
\text { Temperature }\left({ }^{\circ} \mathbf{C}\right)\end{array}$ \\
\hline A & Upward & $4.9 \pm 0.6$ & $690 \pm 35$ \\
B & Upward & $4.3 \pm 0.7$ & $653 \pm 33$ \\
B & Downward & $4.6 \pm 0.9$ & $684 \pm 34$ \\
\hline
\end{tabular}




\section{Appendix C: Experimental Set-up and Excavation}

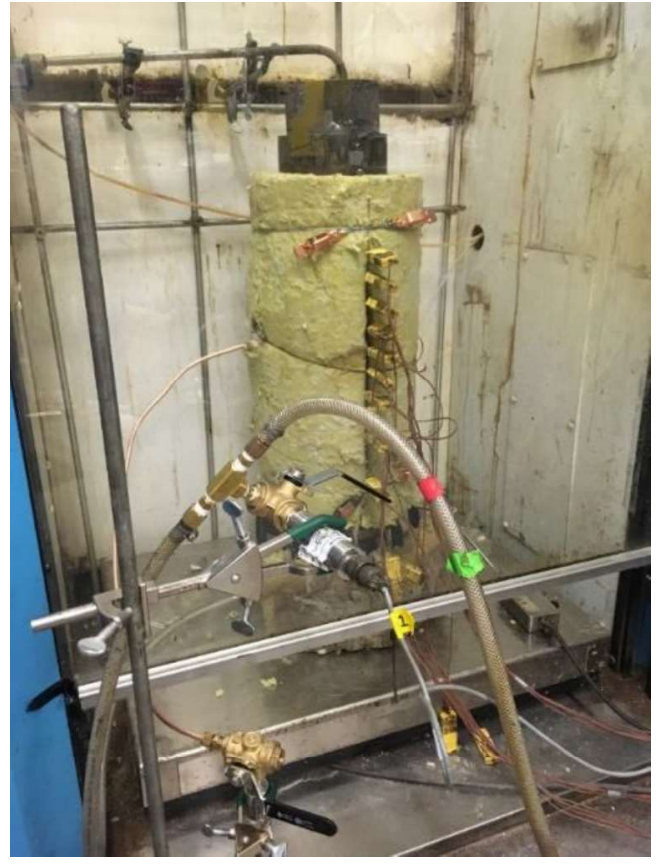

(a)

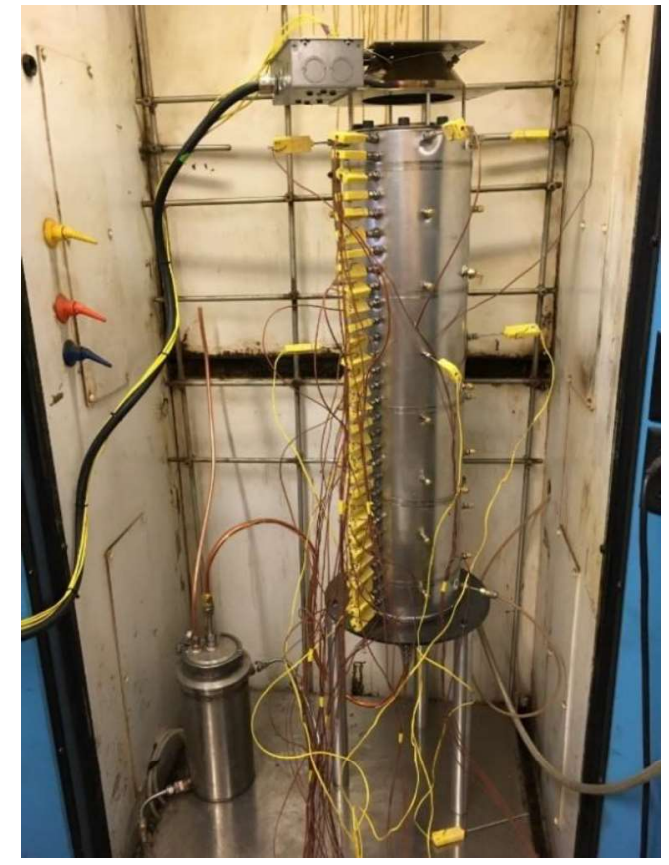

(b)

Figure C-1: (a) Smouldering Column A (Base Case, Parallel 1-3 and Complex: Disconnected Coarse) and (b) Column B (Series 1-4).

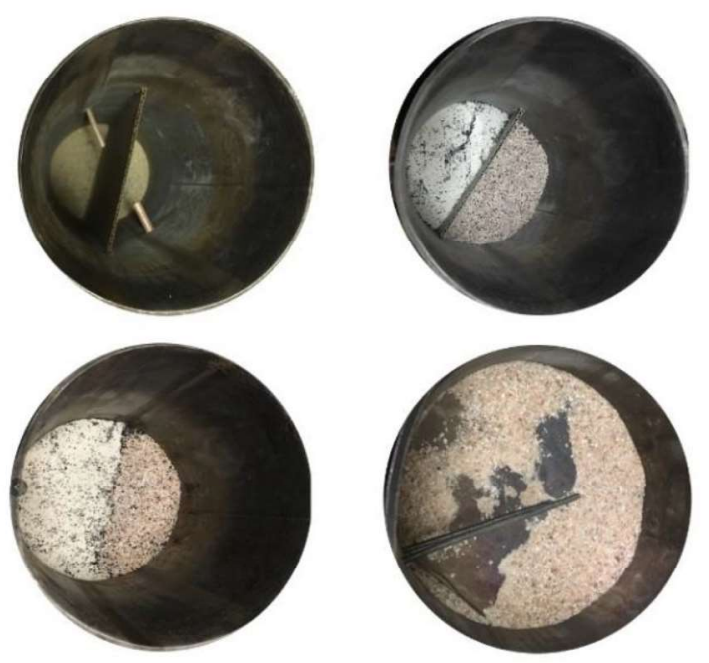

Figure C-2: Loading process for Parallel 1-3 and Complex: Disconnected Coarse, the coarse layer (yellow particles) and fine layer (white particles) were divided by a cardboard sheet, which was taken out before the smouldering. 
Figure C-3a shows that much GAC was left in the fine layer after smouldering in Parallel 1, confirming no obvious smouldering reaction in the fine layer; Figure C-3b also shows much GAC was left in the fine layer whereas the base of fine layer was clean in posttreatment Parallel 2, which was in accordance with the temperature profile, showing that the smouldering was ignited in the base of fine layer but unable to propagate upward successfully due to the limited air flux. Figure C-3c shows that fine layer was clean in the bottom whereas more remaining GAC was found as the height of fine layer after smouldering in Parallel 3, supporting the conclusion that the smouldering started and propagated but did not complete on the top of fine layer due to heat losses. Figure C-3d shows unburnt GAC near the edge of the fine layer in Complex: Disconnected Coarse, revealing the smouldering near the wall with limited air flux was insufficient to cover heat losses to sustain the stable smouldering reaction.

(a)
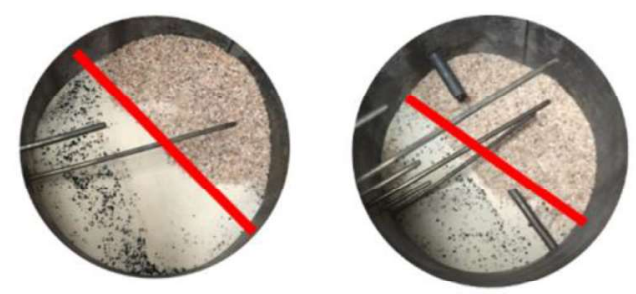

(b)
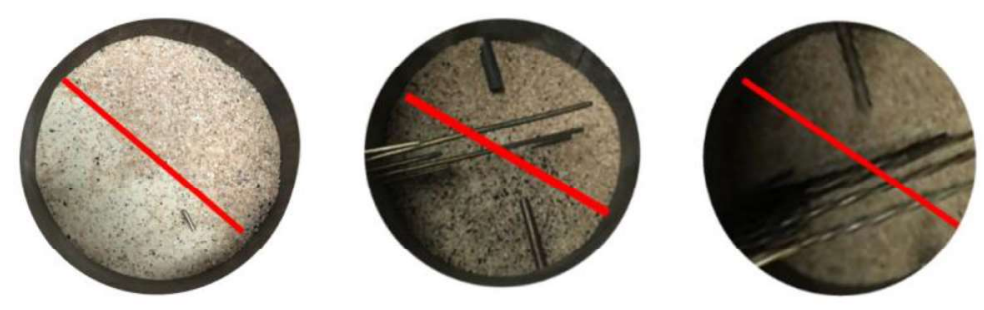
(c)

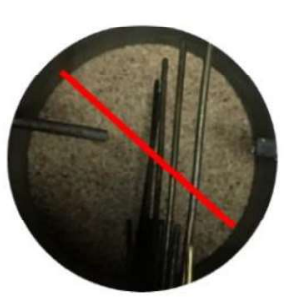

Bottom (clean)

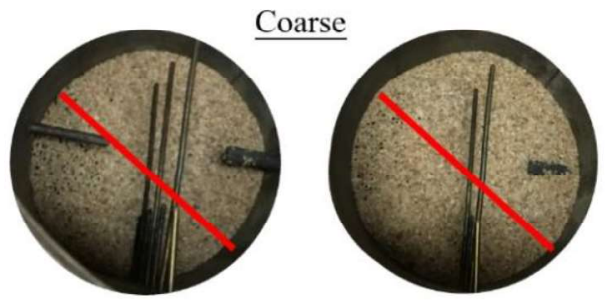

$\underline{\text { Fine }}$

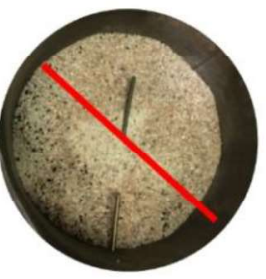

Top (Unburned)

(d)

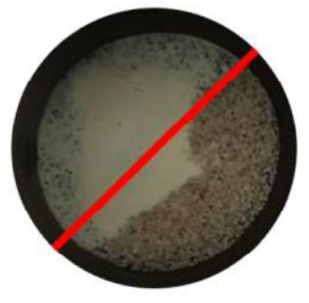

Bottom (clean)
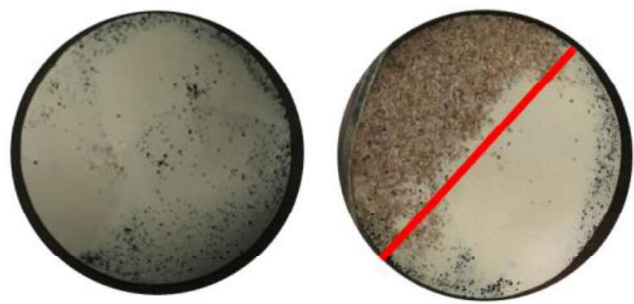

Top (Unburned)

Figure C-3: Excavation of (a) Parallel 1, (b) Parallel 2, (c) Parallel 3 and (d) Complex: Disconnected Coarse after smouldering treatment, in which white particles were fine materials and small black particles were unburnt GAC left in the porous media. 


\section{Appendix D: Pressure Changes during Experiments}

Homogeneous 1 (Extra Case) employed a single contaminated layer to explore pressure changes during the smouldering (Column B, upward smouldering, $20 \mathrm{~g} \mathrm{GAC} / \mathrm{kg} \mathrm{Sand,} \mathrm{k}=$ $3.6 \times 10^{-10} \mathrm{~m}^{2}, 74.5 \mathrm{~cm}$ contaminated layer, $5.0 \mathrm{~cm} / \mathrm{s}$ air flux). As shown in Figure D-1, four locations of pressure transducers (in order near TC1, TC9, TC16 and TC23) all got initial pressure values when the air was turned on and there existed the same pressure difference between each pair of adjacent PTs, which was correspond to the energy consumption for air passing through sands. With the evolution of smouldering, pressure started to increase obviously in sequence when smouldering reaction hit PT areas (TC1, TC9, TC16, $\mathrm{TC} 23=200^{\circ} \mathrm{C}$, , red dots and orange lines represent these specific times.

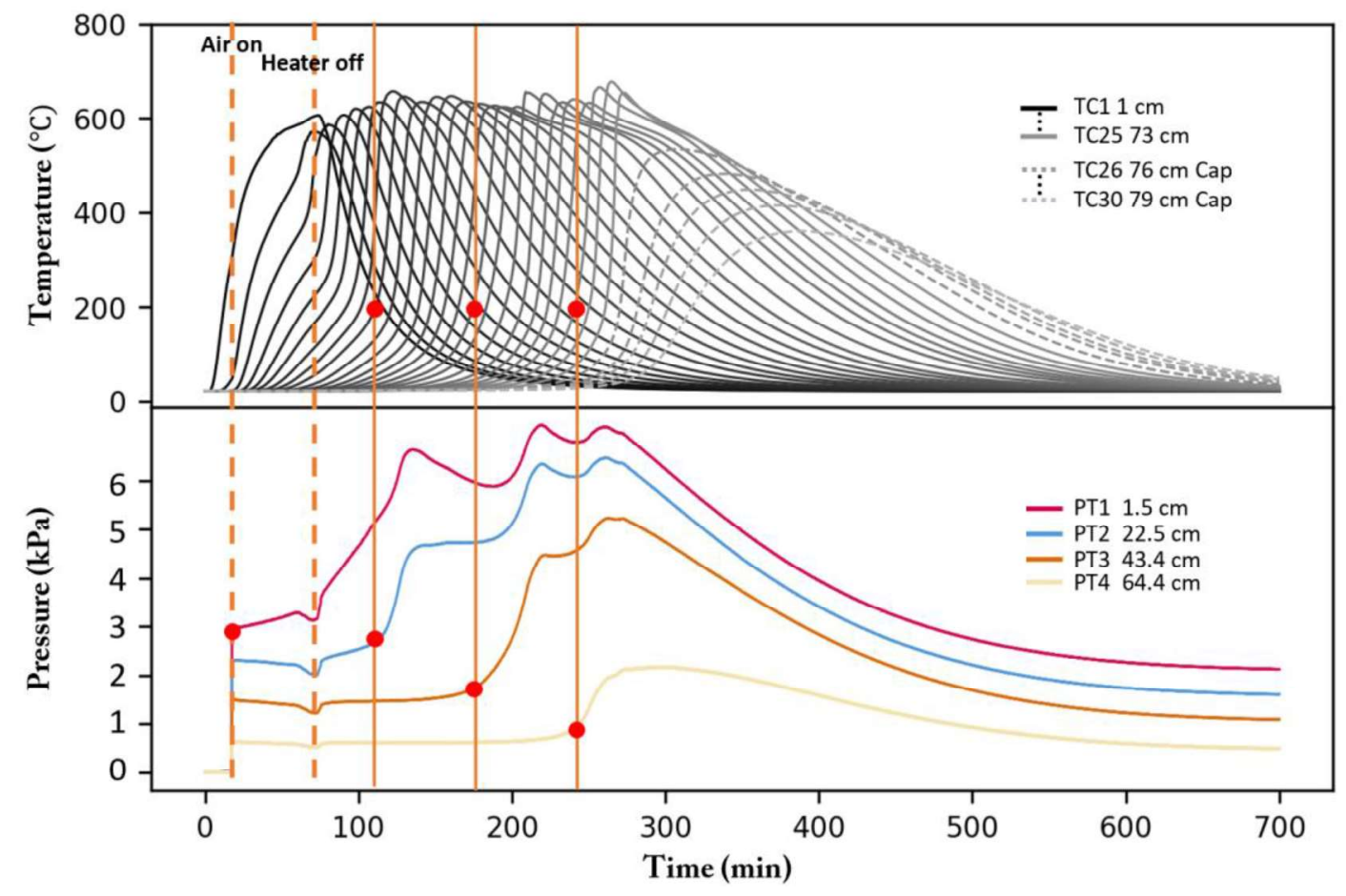

Figure D-1: Temperature and pressure profiles for Homogeneous 1.

After 270 minutes, smouldering in the system was close to complete as evidenced by the peak of last TC in the reaction zone; meanwhile, all pressures started to decrease gradually 
until the whole column temperature went back to ambient, meaning the hot zone was shrinking.

Since air flux was kept constant as $5.0 \mathrm{~cm} / \mathrm{s}$, pressure changes during smouldering were supposed to result from changes of pneumatic conductivity based on Darcy's law:

$$
Q=-K A \frac{d h}{d l}
$$

Where $Q$ is volumetric flow rate $\left(\mathrm{m}^{3} / \mathrm{s}\right), K$ is pneumatic conductivity $(\mathrm{m} / \mathrm{s}), A$ is crosssectional area of porous medium $\left(\mathrm{m}^{2}\right), d h$ is the drop of pneumatic head $(\mathrm{m}), d l$ is the length of sample (m).

Pneumatic conductivity is determined by Equation (3) and permeability is the property of porous media (assumed as a constant value during the smouldering), so it was clear that the substantial decrease of pneumatic conductivity resulted from the increase of kinematic viscosity of air (ratio of dynamic viscosity to density). PT1-PT4 only covered the area from $\mathrm{TC} 1$ to $\mathrm{TC} 23$, therefore all calculations in the following related to the kinematic viscosity and temperature were limited within this area (TC1-TC23; PT1-PT4), which was considered as the system scale.

$$
K=\frac{k \rho g}{\mu}
$$

Where $k$ is intrinsic permeability $\left(\mathrm{m}^{2}\right), \mu$ and $\rho$ are the viscosity $(\mathrm{kg} / \mathrm{m} / \mathrm{s})$ and density $\left(\mathrm{kg} / \mathrm{m}^{3}\right)$ of the fluid passing through porous media and $g\left(\mathrm{~m} / \mathrm{s}^{2}\right)$ is the gravitational acceleration. 
The density of dry air can be calculated using the ideal gas law, expressed as a function of temperature and pressure (Equation 4), where air inside the system was assumed as one same condition based on harmonic average temperature and pressure.

$$
\rho_{\text {dry air }}=\frac{P}{R . T}
$$

Where $\rho_{\text {dry air }}$ is density of dry air $\left(\mathrm{kg} / \mathrm{m}^{3}\right), P$ is air pressure $(\mathrm{Pa}), R$ is specific gas constant for dry air, $287.05(\mathrm{~J} / \mathrm{kg} / \mathrm{K})$ and $T$ is temperature $(\mathrm{K})$.

The calculation of viscosity was according to Sutherland's viscosity Law (1893), where the viscosity of air is the function of temperature:

$$
\mu=\mu_{0}\left(\frac{T}{T_{0}}\right)^{3 / 2} \frac{T_{0}+S}{T+S}
$$

Where $T$ is the average static temperature of air $(\mathrm{K}), \mu_{0}$ is a reference value, $1.79 \times 10^{-5}$ $\mathrm{kg} / \mathrm{m} / \mathrm{s}, \mathrm{T}_{0}$ is a reference temperature, $273.11 \mathrm{~K}$ and $S$ is an effective temperature $(\mathrm{K})$, called the Sutherland constant, which is characteristic of the gas, here $S=110.56 \mathrm{~K}$.

Figure D-2 shows real-time changes of dynamic viscosity, density and kinematic viscosity of air during the smouldering process. It reveals that all above parameters changed as the function of system temperature, and it is worthwhile to note that average system temperature reached the highest value around the same time when TC23 peaked, which means the hot zone actually kept expanding with an increasing thickness, even though simultaneously a part of post-treatment zone was cooling down. With the development of smouldering, average air density gradually decreased whereas average dynamic viscosity increased at the same time which led to the obvious rise of air kinematic viscosity in the 
system; when smouldering was complete (average system temperature peaked), all density and viscosity values reached their own extreme point and went back to ambient.

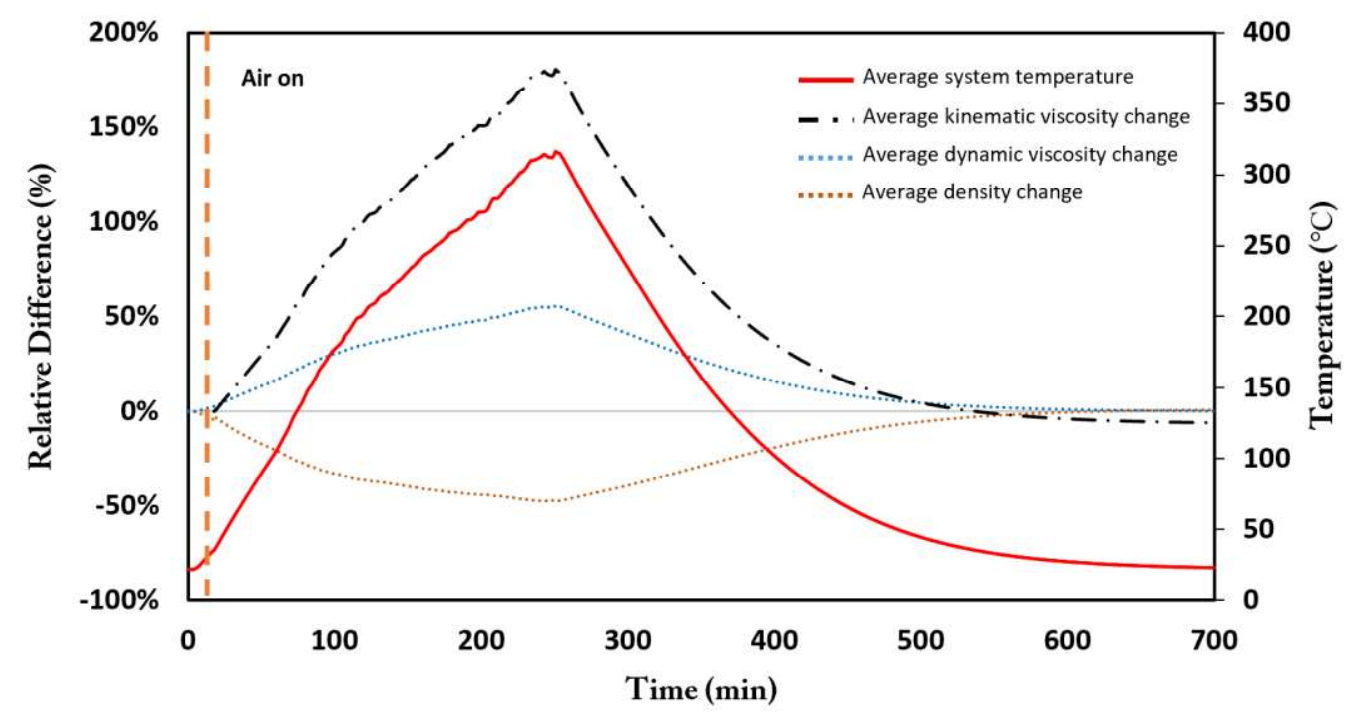

Figure D-2: Changes of the dynamic viscosity, density and kinematic viscosity of air during smouldering process for Homogeneous 1.

Figure D-3 illustrates the relationship among changes of pressure drop, pneumatic conductivity and average system temperature, which confirms that temperature-related air property change (average density and viscosity) resulting in the decrease of pneumatic conductivity was the main impact on pressure changes during the smouldering process. That peaks of average system temperature and pressure drop being apart a little bit maybe was due to the fact that the average system temperature was overestimated since only temperatures in the center line of the column were recorded, which had a better thermal insulation than areas near the wall. 


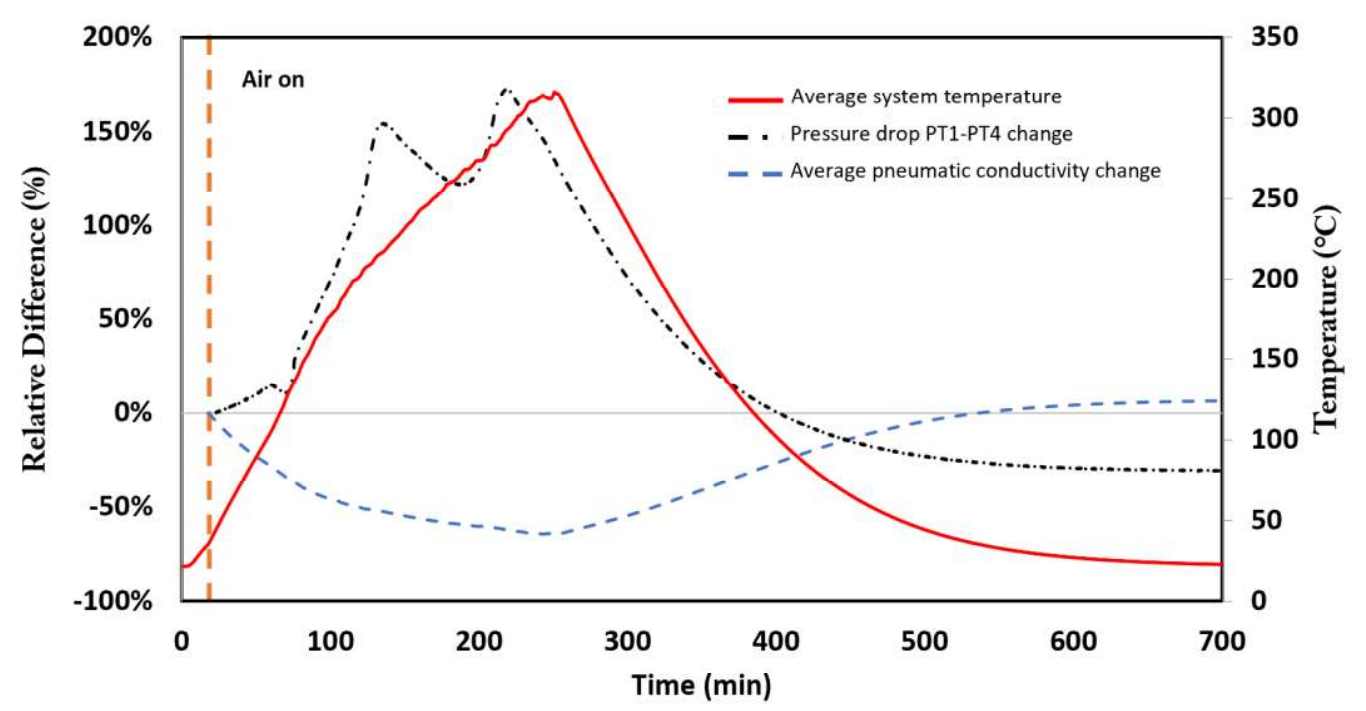

Figure D-3: Changes of pressure drop and pneumatic conductivity during smouldering process for Homogeneous 1.

As shown in Figure D-1, there were several unsystematic dips during the increase of pressure, some dips occurred in all locations at the same time, but some dips only were observed in one or two pressure measurement areas. Figure D-3 shows that these pressure dips were not correspond to changes of pneumatic conductivity which assumed intrinsic permeability as a constant value during the smouldering. Since pressure gradient is supposed to be inversely proportional to the pneumatic conductivity under the condition of constant air flux, fixed permeability assumption may be the problem leading to the deviation during these dips; in other words, permeability may change during the smouldering because it removed GAC from the porous media, creating local preferential paths (causing lower permeability) that temporally reduced the pressure gradient. Nevertheless, the continuing smouldering kept expanding the hot zone and increased the average temperature that rebounded the pressure gradient in the system.

Homogeneous 2 (Series 1) with a lower permeability sand layer was also conducted. As shown in Figure D-4, pressure changes presented a similar trend as Homogeneous 1, where 
the air pressure started to increase when the smouldering reaction reached each height of four PTs and began to gradually reduce after the completion of smouldering. Due to lower permeability ( $3 \%$ of Homogeneous 1 ), the pressure value was obviously higher in Homogeneous 1 than Homogeneous 2 (1 order of magnitude higher).

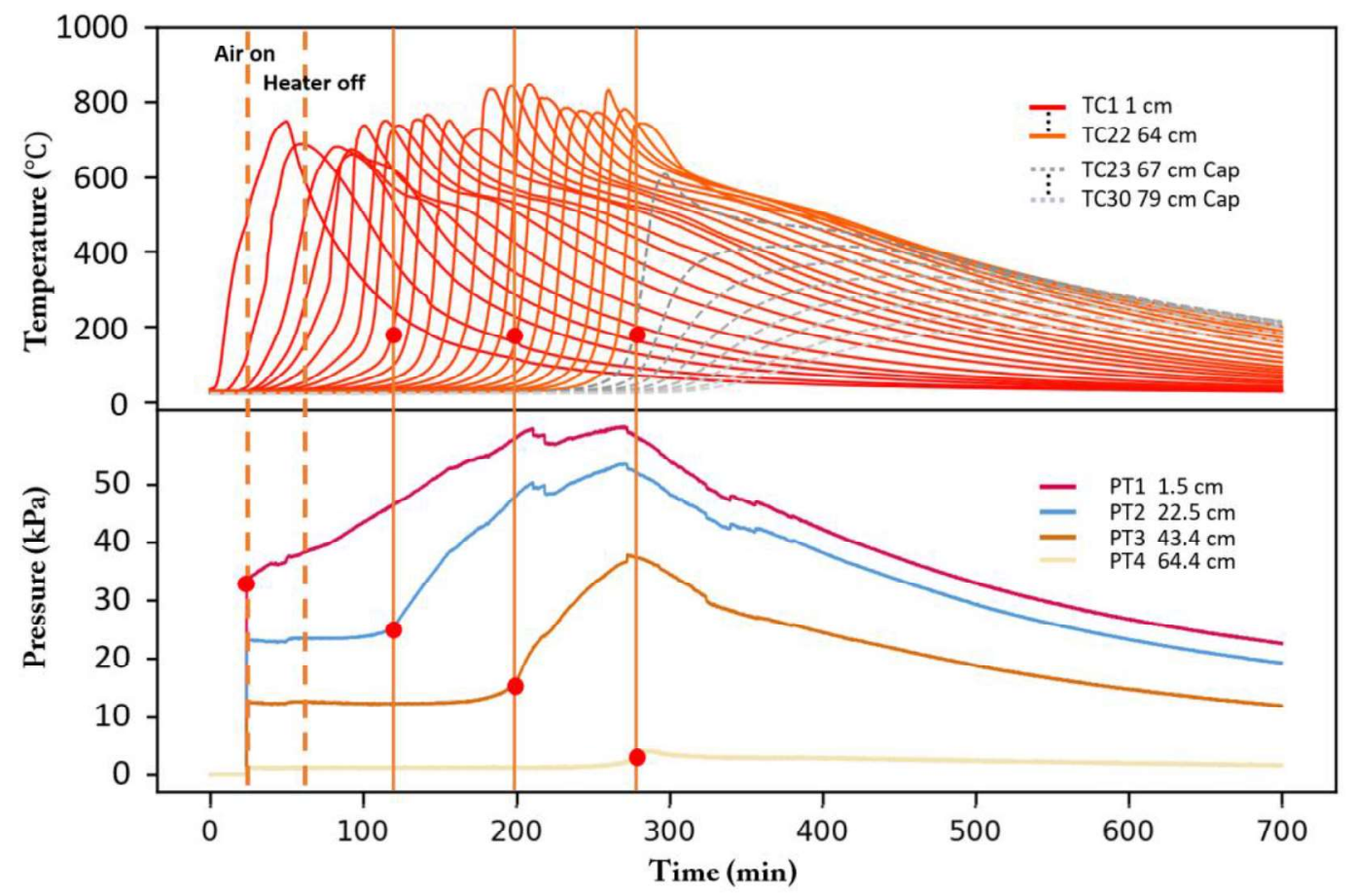

Figure D-4: Temperature and pressure profiles for Homogeneous 2.

Compared with Homogeneous 1, the pressure profile is much smoother in Homogeneous

2. The reason is that porous media used in Homogeneous 1 were directly from \#12ST Silica Sand, whose particle sizes ranged from $0.15 \mathrm{~mm}$ to $2.00 \mathrm{~mm}$ (effective size $=0.88 \mathrm{~mm}$ ); whereas Homogeneous 2 used Sieved Fine, whose particle sizes were only from $0.125 \mathrm{~mm}$ to $0.250 \mathrm{~mm}$. Therefore, it reveals that the wide particle range or low uniformity was more likely to cause pressure fluctuations during the smouldering, which may be due to the fact that microcosmic heterogeneity in the low uniform porous media helped the development of local preferential pathways. 
Figure D-5 illustrates the relationship between changes of pressure drop and pneumatic conductivity and average system temperature for Homogeneous 2, all of which reached their own extreme point around the same time, similar as Homogeneous 1, confirming the above conclusion.

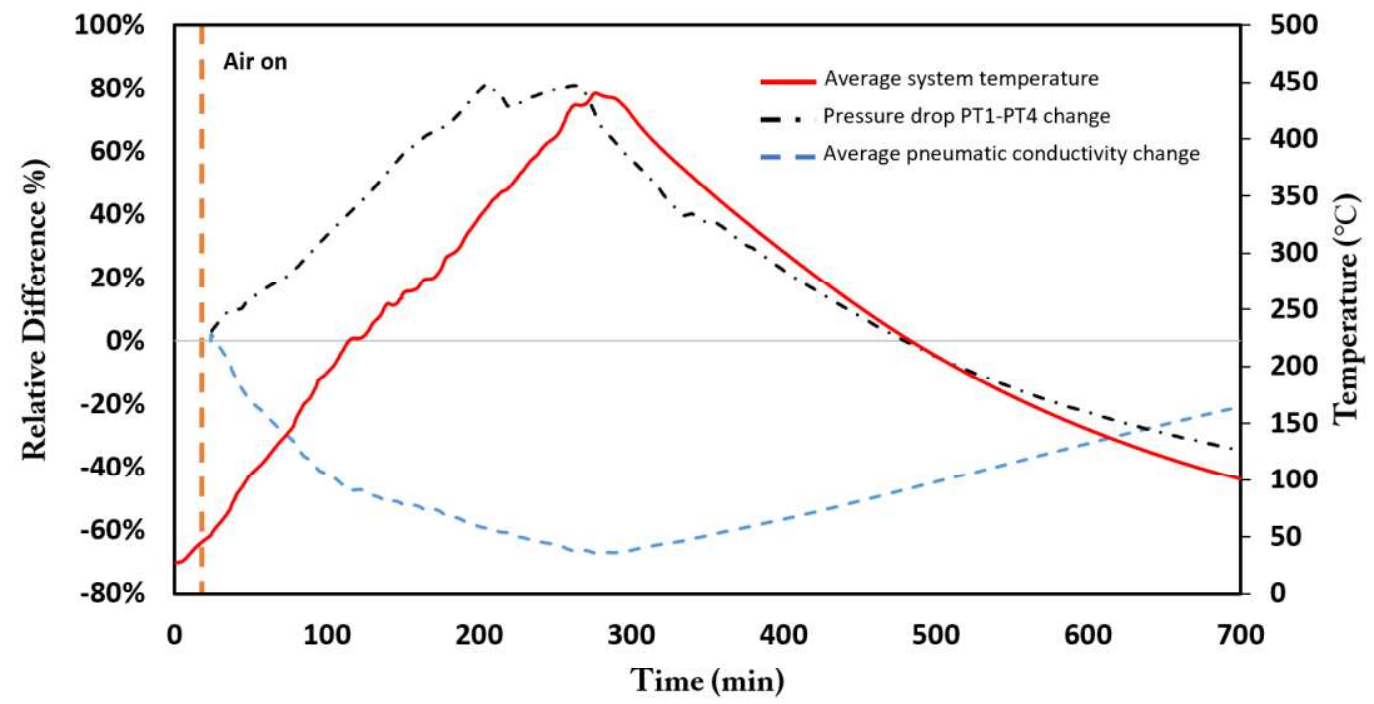

Figure D-5: Changes of pressure drop and pneumatic conductivity during smouldering process for Homogeneous 2.

As shown in Figure D-6, Heterogeneous 1 (Series 2) and Heterogeneous 2 (Series 4) were employed to explore the vertical pressure performance in the heterogeneous situation, where smouldering first passed through the coarse layer and then propagated into the fine layer. In these two pressure profiles, some lines (e.g., PT1 and PT2) were overlapping because the pressure drop within the coarse layer was negligible compared with fine layer.

The permeability of fine layer was around one order of magnitude higher in Heterogeneous 1than Heterogeneous 2, so it is clear to see that the air pressure was also almost one order of magnitude higher in Heterogeneous 2 due to the same mixture height and same air flux. Besides, as shown in profile, most of the pressure increase occurred in the fine layer, because fine layer contributed to the major part of initial pressure gradient in the whole 
system, whose change was more significant compared to the coarse layer with a small proportion of pressure gradient. And compared with Heterogeneous 1, pressure increase was more gentle during the smouldering in the fine layer in Heterogeneous 2, which can be explained by the lower peak temperature in this fine layer; lower peak temperature meant a slower increase rate of average system temperature, therefore the change of kinematic viscosity was also mild which further decreased the pressure increase rate.

(a)

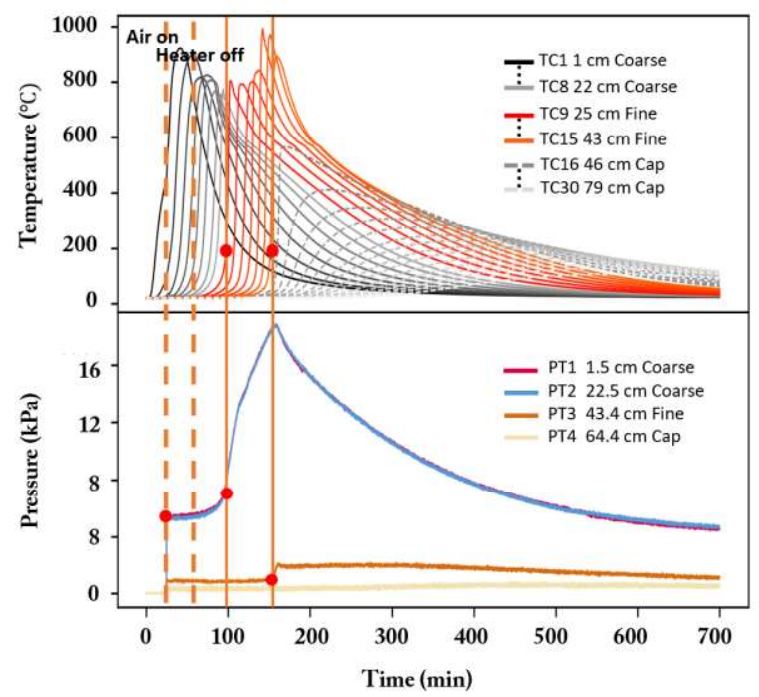

(b)

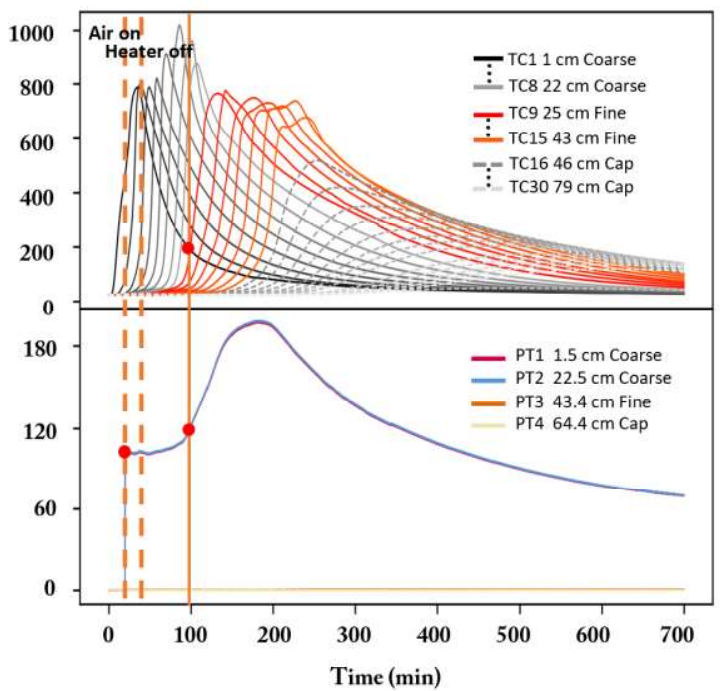

Figure D-6: Temperature and pressure profiles for Heterogeneous tests: (a) Heterogeneous 1 and (b) Heterogeneous 2.

Heterogeneous 3 (Parallel 1) and Heterogeneous 4 (Parallel 3) were used to explore the horizontal pressure performance in the heterogeneous situation, where a $39.5 \mathrm{~cm}$ coarse zone $\left(\mathrm{k}=1.3 \times 10^{-9} \mathrm{~m}^{2}\right)$ and a $39.5 \mathrm{~cm}$ fine zone $\left(\mathrm{k}_{1}=1.9 \times 10^{-11} \mathrm{~m}^{2} ; \mathrm{k}_{3}=4.5 \times 10^{-10} \mathrm{~m}^{2}\right)$ were put in parallel, PT2 and PT3 were inserted into the center of each zone at the same height.

As shown in Figure D-7, it is clear that pressures were almost the same at one height either in the coarse zone or fine zone, because the existence of pressure difference would lead to 
the cross-zone horizontal airflow, which was negligible in the strong vertical upward convective situation. Due to the similar pressure gradient in both coarse and fine layer, the ratio of airflow rates in two zones should be inversely proportional to the permeability ratio.

There was one short period in both Heterogeneous 3 and Heterogeneous 4 when pressure of the coarse sand was higher than fine zone, which may be explained by a higher average system temperature in the coarse zone than fine zone, resulting in a more obvious increase of kinematic viscosity thus more significant pneumatic conductivity decrease in the coarse zone than fine zone.

(a)

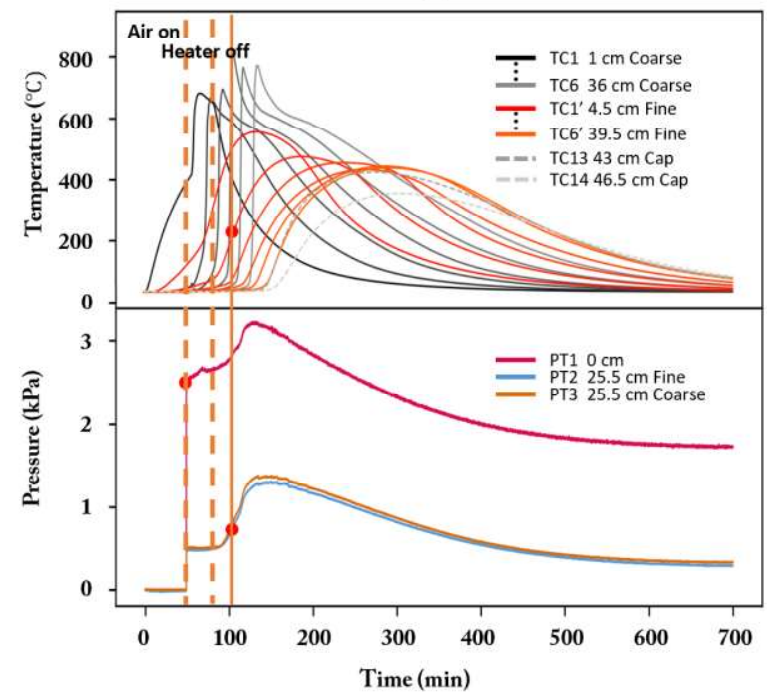

(b)

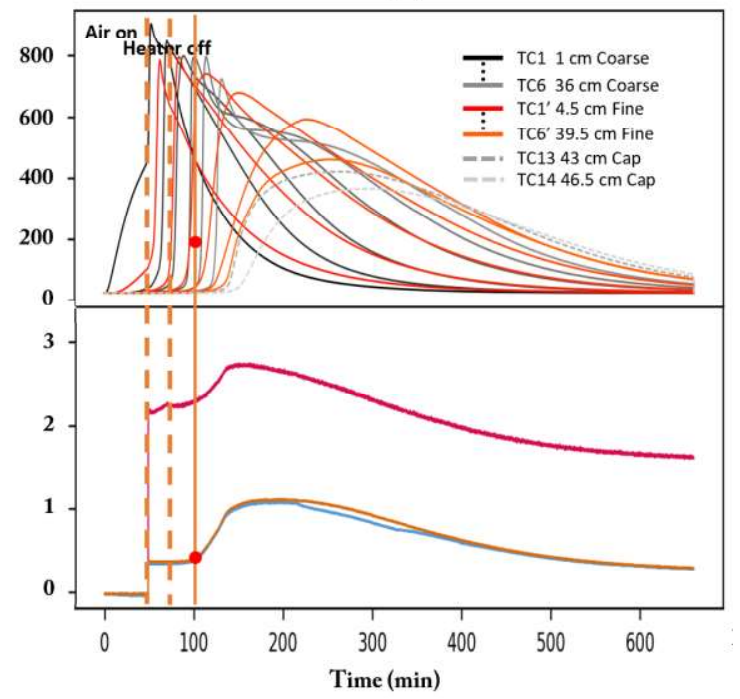

Figure D-7: Temperature and pressure profiles for Heterogeneous tests: (a) Heterogeneous 3 and (b) Heterogeneous 4.

Heat Test without GAC smouldering was carried out to explore the pure temperature effect on the air pressure $\left(90.0 \mathrm{~cm}\right.$ tall column, convective heater, $\mathrm{k}=1.3 \times 10^{-9} \mathrm{~m}^{2}, 11.0 \mathrm{~cm} / \mathrm{s}$ airflow rate,). As shown in Figure D-8, in this case, air was injected into the column through the convective heater from the beginning, and airflow continuously transferred the heat from coils to the sand. The temperature of the sand at different heights reached the plateau 
when the balance was reached among the heat gained, stored and lost. After 260 minutes, the convective heater was turned off, then sand in the low area were gradually cooling down due to the injected cold airflow; whereas the temperature of high area kept similar and even increased a bit because of the energy supplied from below hot zone. After 420 minutes, all areas started to cool down, which meant the hot zone was shrinking. Besides, compared with smouldering pressure profiles that had many dips, the pressure changes of the heat test were smooth, which confirmed that dips of pressure change resulted from the smouldering with the GAC removal (permeability changes).

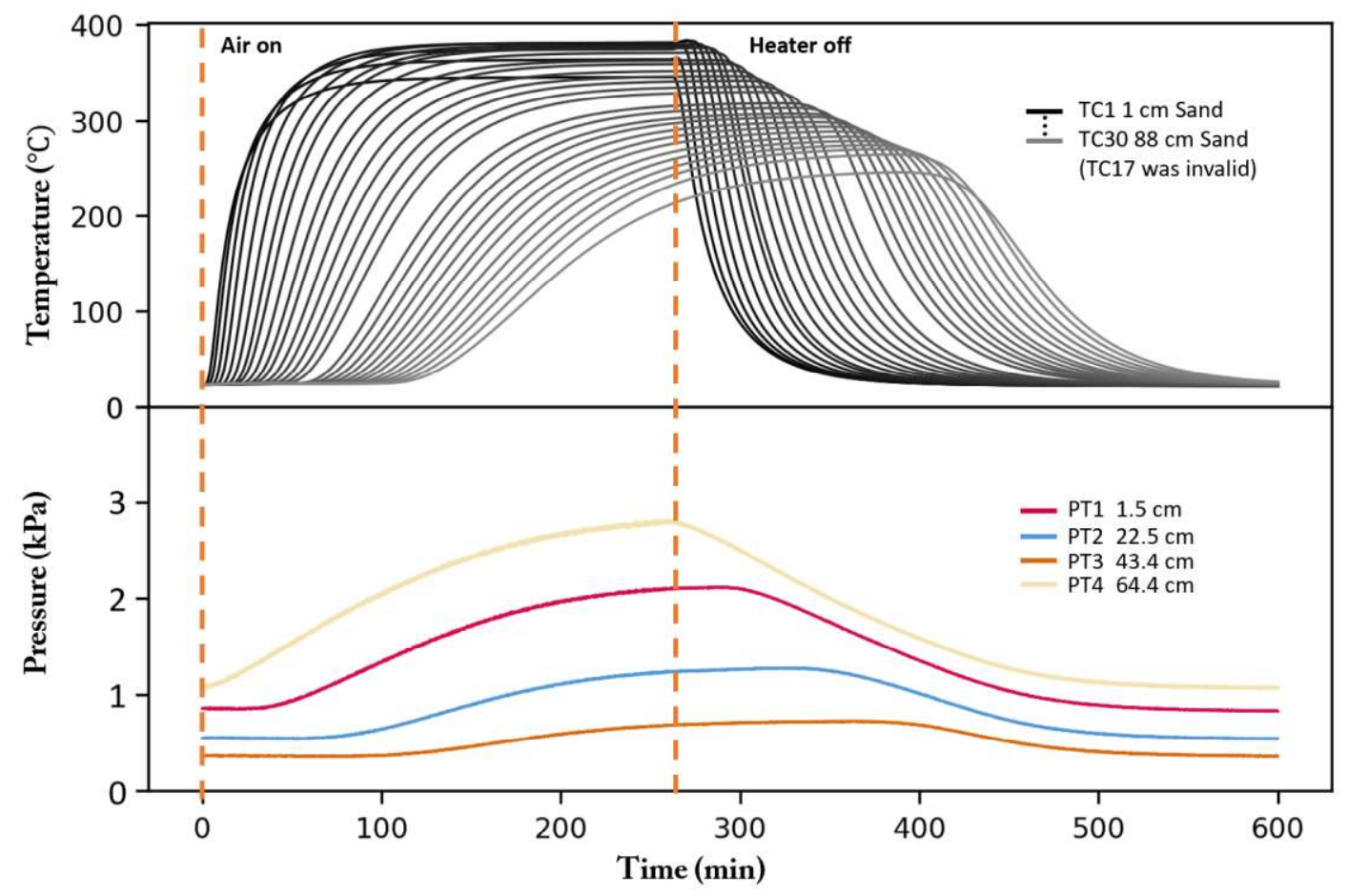

Figure D-8: Temperature and pressure profiles for Heat Test.

Pressure profile shows different pressure behaviours from other smouldering tests, where pressures did not decrease at the same time when the heater was turned off. This behaviour can be explained by the expanding of hot zone, when the heater was turned off, the hot zone had not fully developed until it reached the top of the column; therefore even though 
the average system temperature at the low height was cooling down, average temperature above other pressure transducers may be not, where the hot zone was expanding into their area, keeping decreasing the pneumatic conductivity until the hot zone reached the top. Figure D-9 shows different times of the bottom values of pneumatic conductivity of areas at different heights which correspond to different pressure drop peaks, where the corresponding target area of pneumatic conductivity was above each pressure transducer following the airflow direction until the top of the column, and the pressure drop was actually the reading of each pressure transducer since the outlet pressure was zero in the heat test.

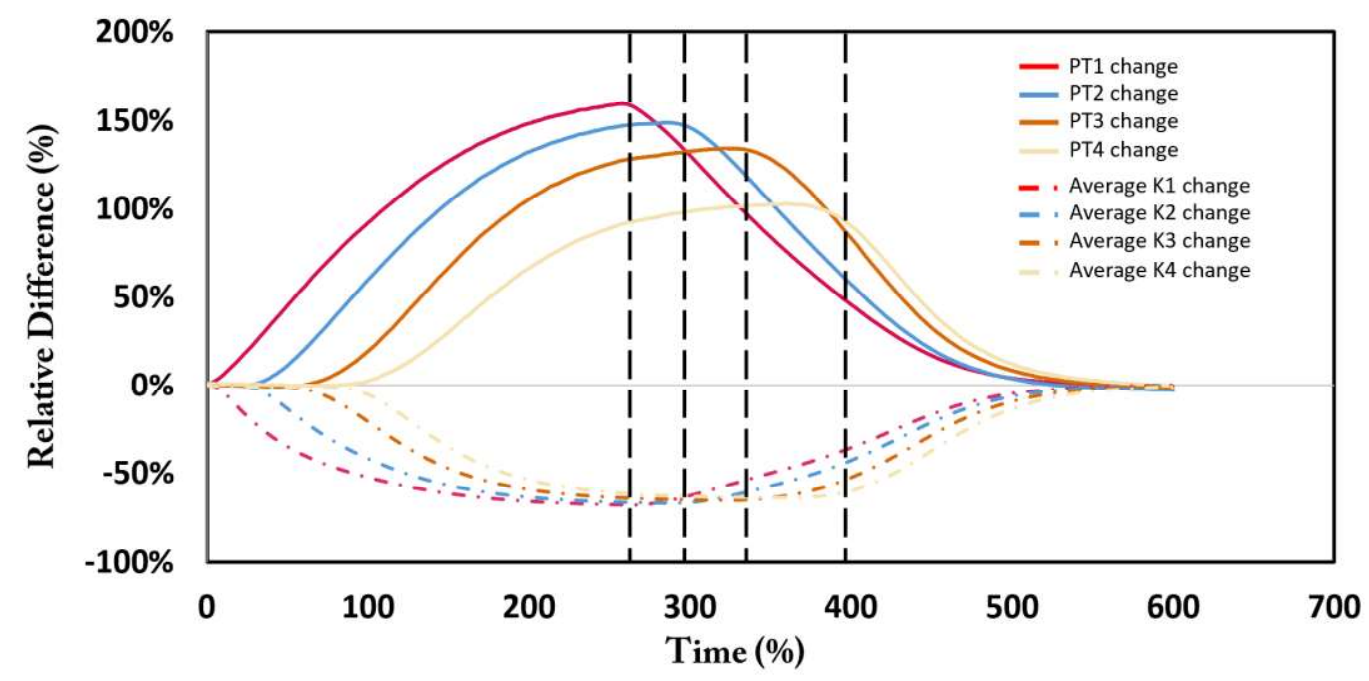

Figure D-9: Changes of pressures and pneumatic conductivity for Heat Test. 


\section{Appendix E: Summary of Principles of ISSM}

ISSM couples a three-dimensional, multi-phase DNAPL flow model, with a combustion reaction expansion model, whose procedure is summarized in Figure E-1.

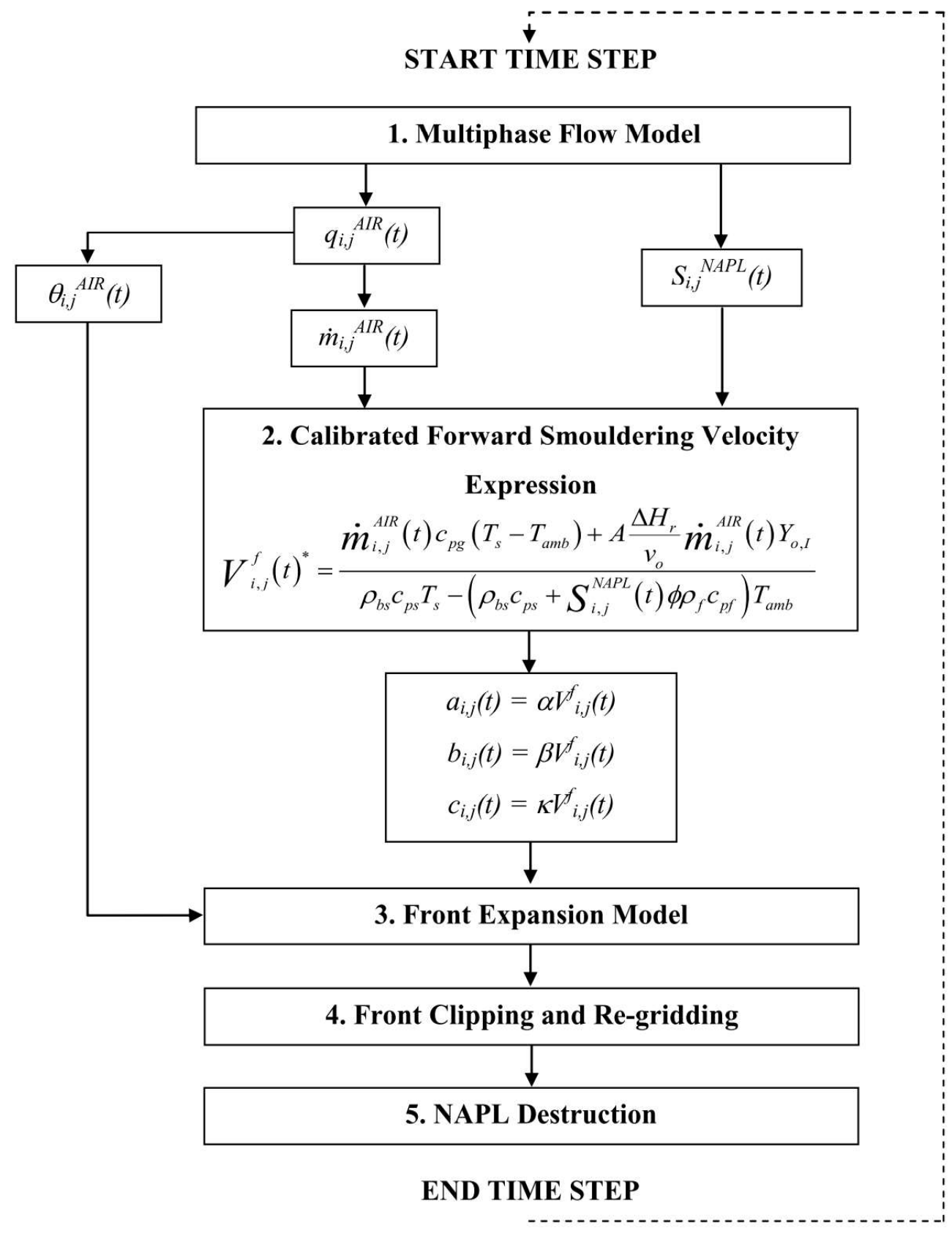

Figure E-1: Process flow diagram for the ISSM. 
Here, DNAPL three-dimensional model first solves the air flux (i.e., at each node, $i, j$ ) for two immiscible fluids inside the porous media to get values of local air mass flux, $\dot{m}_{i, j}^{A I R}(t)$, via the continuity equations and Darcy's law. As for two dimensional scenarios, the wetting and non-wetting phase mass conservation equations are obtained:

$$
\begin{array}{cc}
\frac{\partial}{\partial x_{i}}\left[\frac{k_{i j} k_{r, W}}{\mu_{W}}\left(\frac{\partial P_{W}}{\partial x_{j}}+\rho_{W} g \frac{\partial z}{\partial x_{j}}\right)\right]-\emptyset \frac{\partial S_{W}}{\partial t}=0 & i, j=x, y \\
\frac{\partial}{\partial x_{i}}\left[\frac{k_{i j} k_{r, N}}{\mu_{N}}\left(\frac{\partial\left(P_{C}+P_{W}\right)}{\partial x_{j}}+\rho_{N} g \frac{\partial z}{\partial x_{j}}\right)\right]+\emptyset \frac{\partial S_{N}}{\partial t}=0 & i, j=x, y
\end{array}
$$

Where $k_{i j}$ is the second order tensor defining the intrinsic permeability of porous media; $k_{r, W}$ and $k_{r, N}$ are relative permeabilities of the wetting and non-wetting phase; $\mu_{W}$ and $\mu_{N}$ are viscosities of the wetting and non-wetting phase; $P_{W}, P_{N}$ and $P_{C}$ are wetting, nonwetting phase pressures and capillary pressure, where $P_{N}=P_{C}+P_{W} ; \rho_{W}$ and $\rho_{N}$ are densities of the wetting and non-wetting phase; $S_{W}$ and $S_{N}$ are saturation of wetting and non-wetting phase. $g$ is gravitational acceleration; $\emptyset$ is the porosity; $t$ is time; and $x$ and $y$ are the spatial coordinates.

Equation 6 and 7 are solved numerically by a seven-point, node centered, finite difference scheme, with second-order accurate spatial operators and a first-order accurate derivative. Intrinsic permeabilities are calculated by using harmonic averages, and relative permeabilities are defined using saturations at the upstream node. Full Newton-Raphson iteration and a modified ORTHIM routine are employed to address the non-linear nature of the equations. Air fluxes solved from multiphase flow model are used to calculate the air mass fluxes $\dot{m}_{i, j}^{A I R}(t)$ by considering the density of the air. 
Then both $\dot{m}_{i, j}^{A I R}(t)$ and NAPL saturation, $S_{i, j}^{N A P L}(t)$ are used for the calculation of the velocity of local combustion reaction expansion, $V_{i, j}^{f}$. In previous studies, the analytical model results show a good fit with experimental results for predicting the smouldering front velocity by adjusting key parameters related to the effective heat of smouldering and stoichiometry. Therefore a similar adjustment for ISSM is:

$$
V_{i, j}^{f}=\frac{\dot{m}_{i, j}^{A I R}(t) \cdot c_{p g} \cdot\left(T_{s}-T_{a m b}\right)+A \cdot \frac{\Delta H_{r}}{v_{O}} \cdot \dot{m}_{i, j}^{A I R}(t) \cdot Y_{O, I}}{\left(\rho_{b s} \cdot c_{p s} \cdot T_{s}-\left(\rho_{b s} \cdot c_{p s}+S_{i, j}^{N A P L}(t) \cdot \emptyset \cdot \rho_{N A P L} \cdot c_{p N A P L}\right) \cdot T_{a m b}\right)} \quad i, j=x, y
$$

Where $\dot{m}_{i, j}^{A I R}(t)$ is the air mass flux; $c_{p g}, c_{p s}$ and $c_{p N A P L}$ are the specific heat constants for the gas, solid and NAPL phases respectively, where the temperature effects on the heat constant are not explored here; $T_{s}$ and $T_{a m b}$ are peak smouldering temperature and the ambient system temperature; $\rho_{b s}$ and $\rho_{N A P L}$ are the bulk density of the solid and density of the NAPL phase; $\Delta H_{r}$ is the effective heat of smouldering; $v_{O}$ is the oxygen/fuel overall stoichiometric coefficient; $Y_{O, I}$ is the initial mass fraction of oxygen present in the gas phase; finally $A$ presents the calibration parameter in this adjustment determined by the minimum value of Root Mean Square Error between the experimental and numerical results. The threshold of the air flux for successful smouldering is assumed to be $0.5 \mathrm{~cm} / \mathrm{s}$ in the model, even though it has not been experimentally determined. Therefore the comparison between calibrated and experimental results across a range of air fluxes for a given fuel and threshold of air flux for smouldering is used for the calibration experimental set. Based on Hottpad experiments data, the value $A=0.25$ and $\lambda=0.5 \mathrm{~cm} / \mathrm{s}$ were determined to minimize the difference between experimental and model-predicted calibration metrics in this case. 
After that, reaction velocities at each node, $V_{i, j}^{f}$, are used in the reaction expansion model, where a series of "sub-ellipses" are generated along the perimeter to approximate the smouldering reaction expansion. At the end of the time step, the new combustion front is defined as the curve that envelopes all sub-ellipses (Figure E-2).

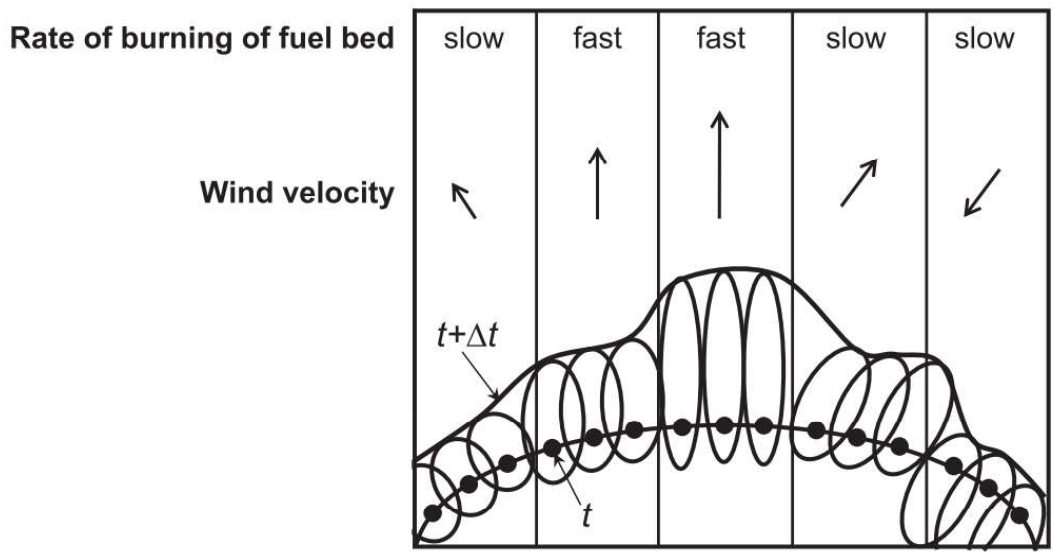

Figure E-2: Illustration of the front expansion model.

At specific time $(t)$, the expansion rate of the combustion perimeter is defined by:

$$
\begin{gathered}
\frac{\partial x}{\partial t}=\frac{a^{2} \cos \theta\left(\frac{\partial y}{\partial s} \cos \theta-\frac{\partial x}{\partial s} \sin \theta\right)+b^{2} \sin \theta\left(\frac{\partial x}{\partial s} \cos \theta+\frac{\partial y}{\partial s} \sin \theta\right)}{\sqrt{\left(\left(a\left(\frac{\partial y}{\partial s} \cos \theta-\frac{\partial x}{\partial s} \sin \theta\right)\right)^{2}+\left(b\left(\frac{\partial x}{\partial s} \cos \theta+\frac{\partial y}{\partial s} \sin \theta\right)\right)^{2}\right)}}+c \cos \theta \\
\frac{\partial y}{\partial t}=\frac{a^{2} \sin \theta\left(\frac{\partial y}{\partial s} \cos \theta-\frac{\partial x}{\partial s} \sin \theta\right)+b^{2} \cos \theta\left(\frac{\partial x}{\partial s} \cos \theta+\frac{\partial y}{\partial s} \sin \theta\right)}{\sqrt{\left(\left(a\left(\frac{\partial y}{\partial s} \cos \theta-\frac{\partial x}{\partial s} \sin \theta\right)\right)^{2}+\left(b\left(\frac{\partial x}{\partial s} \cos \theta+\frac{\partial y}{\partial s} \sin \theta\right)\right)^{2}\right)}}+c \sin \theta
\end{gathered}
$$

Where $x, \mathrm{y}$ are coordinates that defines the combustion front perimeter at time $t$, and $\mathrm{s}$ is the angle between the sub-ellipse and the major axis of the ellipse $(0 \leq \mathrm{s} \leq 2 \pi)$; $\theta$ is the direction of airflow relative to the $x$-axis; $a d t, b d t$ are the half of major and minor ellipse axis respectively; $c d t$ is the distance between ellipse center and the ignition point at the rear focus (Figure E-3). 


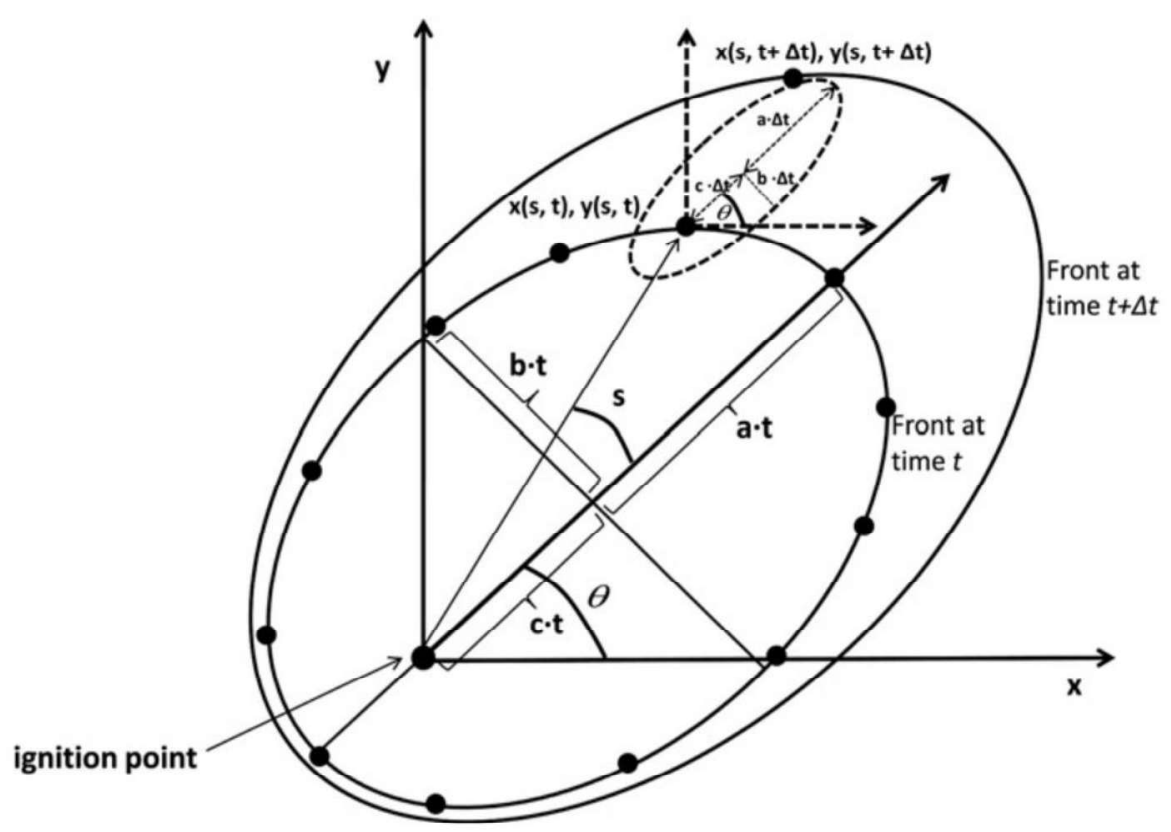

Figure E-3: Schematic of ellipse dimensions and parameter used in Richards' equations to solve for the new combustion front perimeter at time $t+\Delta t$.

The initial condition is a small ellipse at the initial ignition location, which is:

$$
\begin{aligned}
& x(s, 0)=B \cos (s)+x_{i} \\
& y(s, 0)=C \sin (s)+y_{i}
\end{aligned}
$$

Where $B$ and $C$ are the initial dimensions of the ellipse. As shown in Figure E-3, after $\Delta \mathrm{t}$, the distance that a smouldering front moves from the initial location is determined by local variables $a, b, \mathrm{c}$ and $\theta$, which are all functions of $x$ and t. Equations 11 and 12 are solved by forward and finite difference schemes respectively for the spatial derivative (central difference) and temporal derivative (forward difference). The local forward, lateral and opposed expansion rate of combustion front are defined as:

$$
\begin{array}{ll}
V_{i, j}^{f}(t)=a_{i, j}(t)+c_{i, j}(t) & i, j=x, y \\
V_{i, j}^{l}(t)=b_{i, j}(t) & i, j=x, y
\end{array}
$$




$$
V_{i, j}^{o}(t)=a_{i, j}(t)-c_{i, j}(t) \quad i, j=x, y
$$

After rearranging, above three equations can be transferred as following with constants $\alpha, \beta$ and $\kappa$.

$$
\begin{aligned}
& a_{i, j}(t)=\alpha V_{i, j}^{f}(t) \\
& b_{i, j}(t)=\beta V_{i, j}^{f}(t) \\
& c_{i, j}(t)=\kappa V_{i, j}^{f}(t)
\end{aligned}
$$

All parameters of porous media and fluid used in simulations are shown in Table E-1 and E-2:

Table E-1: Parameters Used in the $\mathrm{k}_{\mathrm{rN}}-\mathrm{S}$ Constitutive Relationship Parameter

\begin{tabular}{cll}
\hline & \multicolumn{1}{c}{ Parameter } & Value \\
\hline$k_{r N}^{\text {max }}$ & Maximum relative permeability to the non-wetting phase & Fit \\
$\tau_{d}$ & Drainage non-wetting phase relative tortuosity exponent & Fit \\
$\lambda_{d}$ & Pore size distribution index & $3.41^{\mathrm{a}}$ \\
$S_{O}^{M}$ & Emergence saturation & $0.95^{\mathrm{a}}$ \\
$S_{r}^{k}$ & Residual wetting phase parameter associated with $\mathrm{k}_{\mathrm{rN}}-\mathrm{S}$ & $0.01^{\mathrm{a}}$ \\
$\Delta S_{O}^{* d}$ & Fitting parameter that determines $\mathrm{k}_{\mathrm{rN}}$ when $\mathrm{S}_{\mathrm{o}}=\mathrm{S}_{\mathrm{o}}{ }^{\mathrm{M}}$ & $0.1^{\mathrm{a}}$ \\
\hline
\end{tabular}




\section{Table E-2: Fluid and Porous Media Parameters Used in Simulations}

\begin{tabular}{cll}
\hline & \multicolumn{1}{c}{ Parameter } & \multicolumn{1}{c}{ Value } \\
\hline$c_{p g}$ & Specific heat of the gas & $1100(\mathrm{~J} / \mathrm{kg} / \mathrm{K})^{\mathrm{a}}$ \\
$T_{s}$ & Smouldering temperature & $1030(\mathrm{~K})^{\mathrm{b}}$ \\
$T_{a m b}$ & Ambient Temperature & $293(\mathrm{~K})^{\mathrm{b}}$ \\
$A$ & Forward smouldering velocity calibration parameter & $0.25^{\mathrm{g}}$ \\
$\Delta H_{r}$ & Effective heat of smouldering & $39.4(\mathrm{MJ} / \mathrm{kg})^{\mathrm{b}}$ \\
$v_{o}$ & Overall stoichiometric coefficient & $2.89^{\mathrm{b}}$ \\
$\gamma_{O, I}$ & Mass fraction of oxygen in the gas phase & $\left.0.235(\mathrm{~kg} \mathrm{O})^{2} \mathrm{~kg} \mathrm{gas}\right)^{\mathrm{c}}$ \\
$\rho_{b s}$ & Bulk density of the soil & $1700\left(\mathrm{~kg} / \mathrm{m}^{3}\right)^{\mathrm{d}}$ \\
$c_{p s}$ & Specific heat of the porous media & $1265(\mathrm{~J} / \mathrm{kg} / \mathrm{K})^{\mathrm{c}}$ \\
$\phi$ & Porosity & $0.38^{\mathrm{e}}$ \\
$\rho_{o}$ & Density of the organic liquid & $850\left(\mathrm{~kg} / \mathrm{m}^{3}\right)^{\mathrm{d}}$ \\
$c_{p o}$ & Specific heat of the organic liquid & $1880(\mathrm{~J} / \mathrm{kg} \mathrm{NAPL} / \mathrm{K})^{\mathrm{c}}$ \\
$\alpha+\kappa$ & Local forward velocity constant & $1.0^{\mathrm{f}}$ \\
$\alpha-\kappa$ & Local opposed velocity constant & $0.0^{\mathrm{f}}$ \\
$\beta$ & Local lateral velocity constant & $0.15^{\mathrm{f}}$ \\
$\lambda$ & Air Extinction threshold & $0.5(\mathrm{~cm} / \mathrm{s})^{\mathrm{g}}$ \\
$\sigma$ & Surface tension & $0.033(\mathrm{~N} / \mathrm{m})^{\mathrm{h}, \mathrm{i}}$ \\
$\rho_{a i r}$ & Density of air & $1.204\left(\mathrm{~kg} / \mathrm{m}^{3}\right)^{\mathrm{h} . j}$ \\
$\mu_{a i r}$ & Air viscosity & $1.98 \mathrm{E}-05(\mathrm{~Pa} \mathrm{~s})^{\mathrm{h} . \mathrm{j}}$ \\
$\mu_{o}$ & Organic liquid viscosity & $51.108(\mathrm{~Pa} \mathrm{~s})^{\mathrm{k} .1}$ \\
$k i$ & Mean permeability & Tested \\
$P_{D}$ & Displacement pressure & $258.0^{\mathrm{e}}$ \\
\hline & &
\end{tabular}

${ }^{a}$ Bergman et al. (2011)

${ }^{\mathrm{v}}$ Pironi (2009)

${ }^{c}$ Perry and Green (2008)

${ }^{\mathrm{d}}$ Savron (2016)

${ }^{\mathrm{E}}$ Grant (2005)

${ }^{\mathrm{f}}$ Hasan et al. (2015)

${ }^{\mathrm{g}}$ Rebecca (2016)

${ }^{\text {h }}$ At temperature of 20 degrees

${ }^{\mathrm{i}}$ Pendent Drop Shape Method with an Axisymmetric Drop Shape Analyser (ADSA), Lord et al. (2000)

${ }^{\mathrm{j}}$ Potter (2002)

${ }^{\mathrm{k}}$ At temperature of 24 degrees

${ }^{1}$ Kinsman (2015)

Finally, with the development of smouldering based on front expansion model, NAPL is removed from any nodes that behind the smouldering reaction, whereas the local air flux and NAPL saturation at each node must exceed the calibrated air flux extinction threshold, $\lambda(0.5 \mathrm{~cm} / \mathrm{s}$ here $)$ and saturation extinction threshold, $S^{M I N}(2.4 \%$ here $)$ to make smouldering happen successfully. 


\section{Appendix F: Pressure Changes in ISSM}

Figure F-1 shows that pressure contours at the same height were not uniform, even in the same porous media, which followed the red bending curves in the figure; pressure was higher in the fine layer than coarse layer at the bottom of parallel layers, and then pressure values in two layers started to get closer until the halfway of mixture, above it, pressure in fine layer decreased faster than coarse layer until the top. Combined with air flux values, and it was found that on the upper surface of bottom layer (just below parallel layers), the air flux was slower below the fine layer than coarse layer due to the low permeability zone above, which led to lower pressure gradient based on Darcy's law, creating lower pressure drop below the fine layer over the cross-section of bottom layer. Since pressure started from a plenum with a uniform distribution, the remaining pressure was higher on the upper surface of bottom layer below the fine zone. This was similar as the explanation for the pressure lines on the top of parallel layers.

Based on Figure F-1 and above explanations, it was found that non-uniform pressure distribution occurred during the transition of major permeability change (bottom or top of the contaminated layers), in these areas, air may cross the layers horizontally by following at right angles of curved pressure lines. Due to several major permeability contrasts staggered in Complex: Disconnected Coarse, there were many curved pressure lines, thus air had more chances than Parallel 1 to pass through fine layers to sustain the smouldering reaction. 
(a)

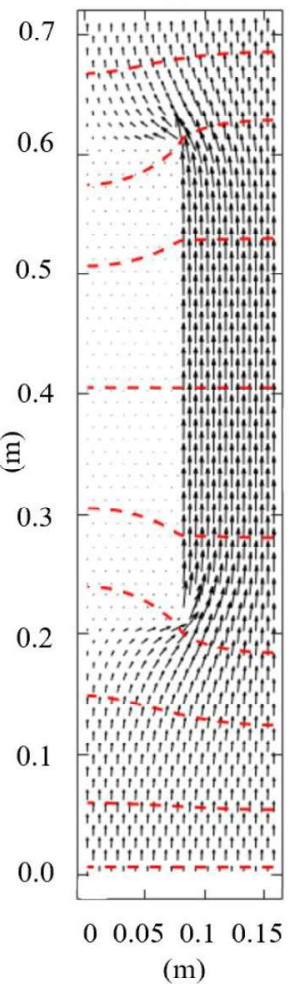

(b)

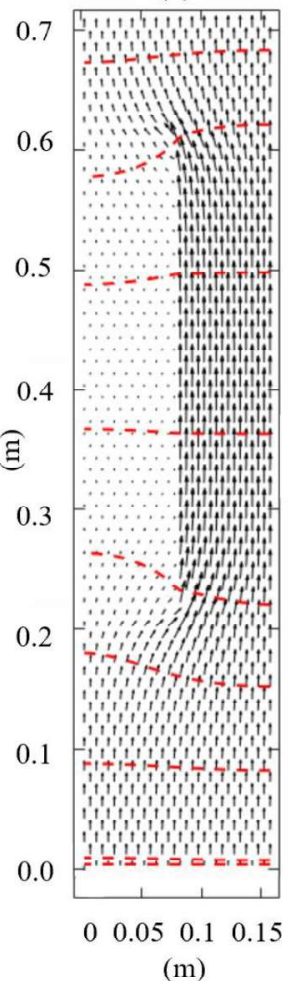

(c)

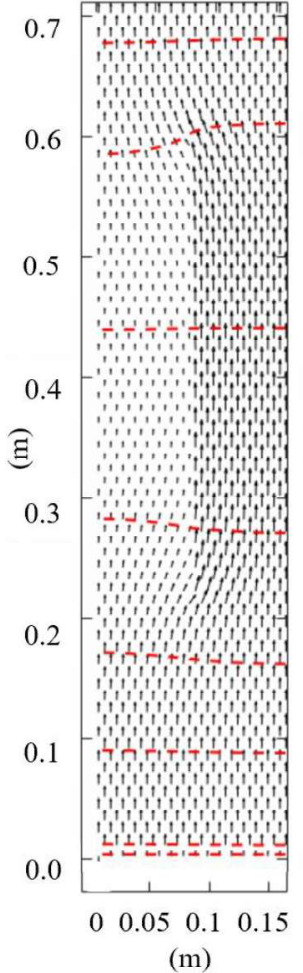

(d)

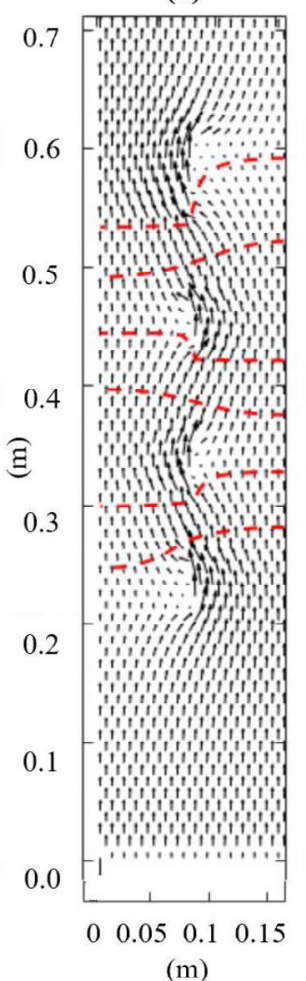

(e)

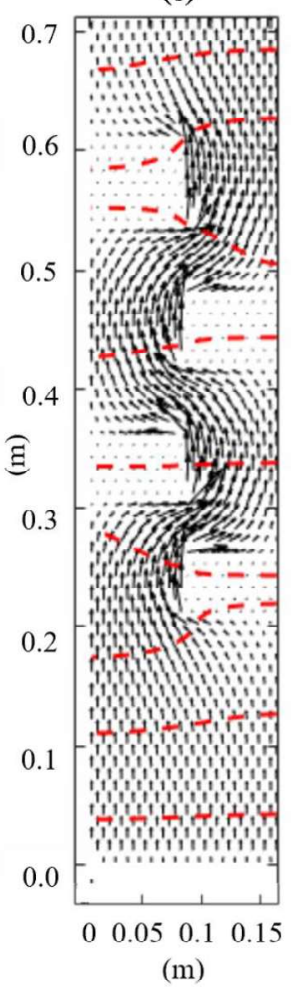

Figure F-1: Air and pressure distributions (red dash-line) in Parallel 1-3 and two Complex cases: (a) Parallel 1, (b) Parallel 2, (c) Parallel 3, (d) Complex: Disconnected Coarse and (e) Complex: Connected Coarse. Each arrow is a velocity vector, with its magnitude expressed by length and direction expressed by orientation. Red dash lines represent pressure contours. 


\section{Appendix G: Comparison of ISSM and Experimental Results}

Results from ISSM, Parallel 1-3 and Complex: Disconnected Coarse tests are compared at different time, in which experimental results were plotted based on the linear interpolation by Python with contours of $600^{\circ} \mathrm{C}$; in ISSM, the pink area represents the presence of contaminants and the yellow area is shown as clean. This work is the first study to validate model simulations to experiments in certain cases. As shown in Figure G-1, arrows in the simulation map the distribution of air flux in the domain (the bigger size, the higher air flux $)$, including a bottom layer (0 - $0.205 \mathrm{~m}$ height), a top clean layer (0.605 - $0.700 \mathrm{~m}$ height) and a contaminated layer (0.205 - $0.605 \mathrm{~m}$ height). The invasion of the yellow area into the pink area represents the smouldering reaction removing the contaminants. The animation of temperature data from experiments starts from the bottom of the contaminated area since there are no temperature data in the bottom clean layer in the experiments, and the area with warm colours within the contour (temperature $>600{ }^{\circ} \mathrm{C}$ ) is the treatment zone, removing the contaminants at this area. 


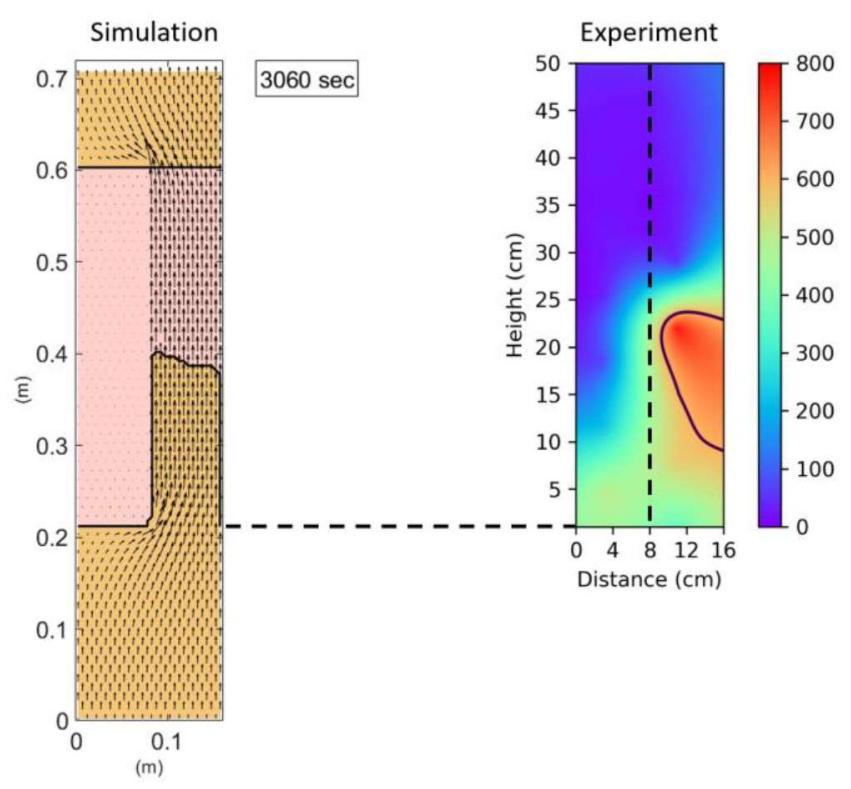

Figure G-1: Comparison of ISSM and Experimental results for Parallel 1 at 3060 seconds.

As shown in the following graphs, ISSM correctly predicted the smouldering behaviour in Parallel 1-2; and Complex: Disconnected Coarse had a similar smouldering behaviour in model and experiment; whereas the experimental results for Parallel 3 were different from what models showed since it did not consider the temperature effect and heat losses. 

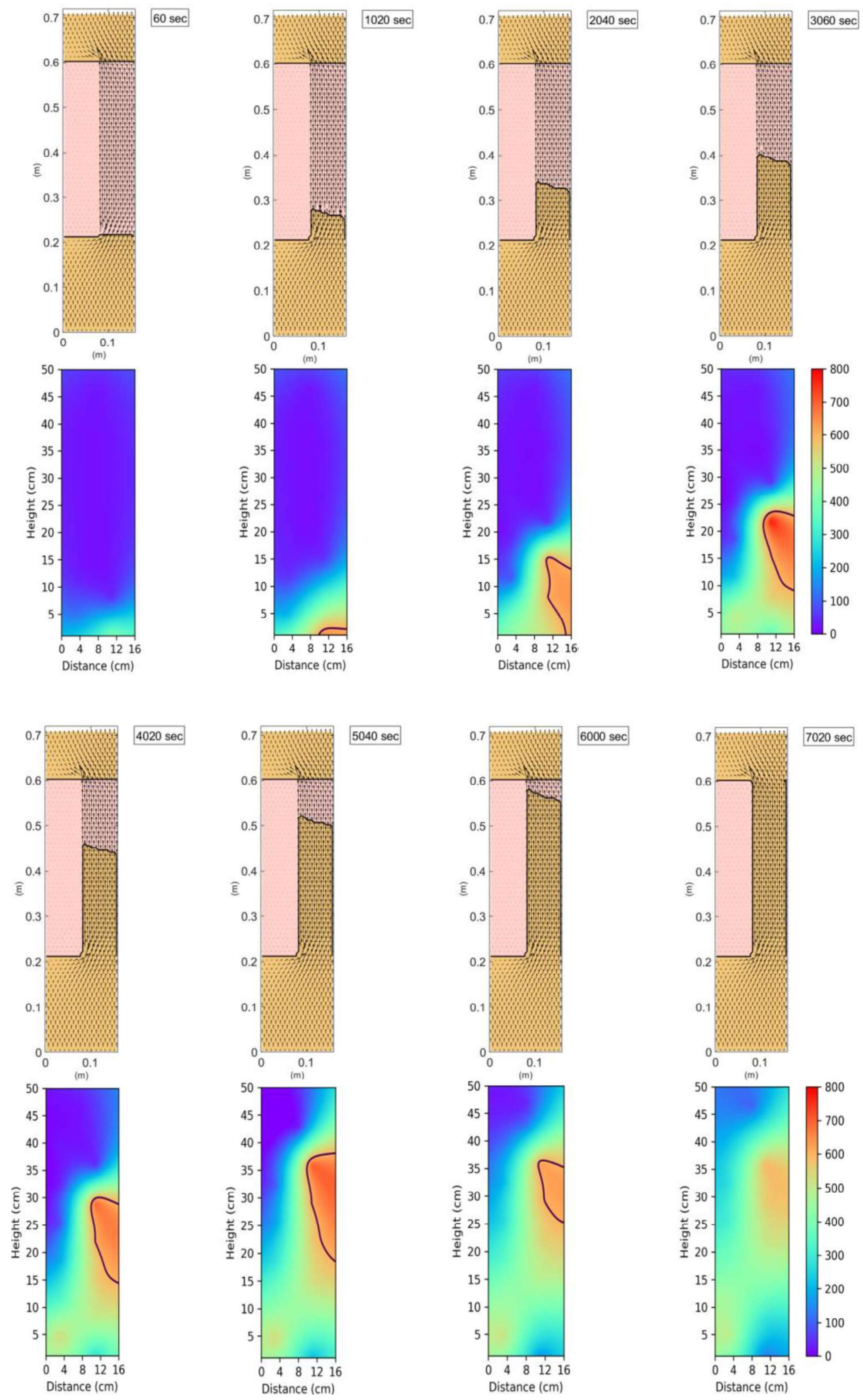

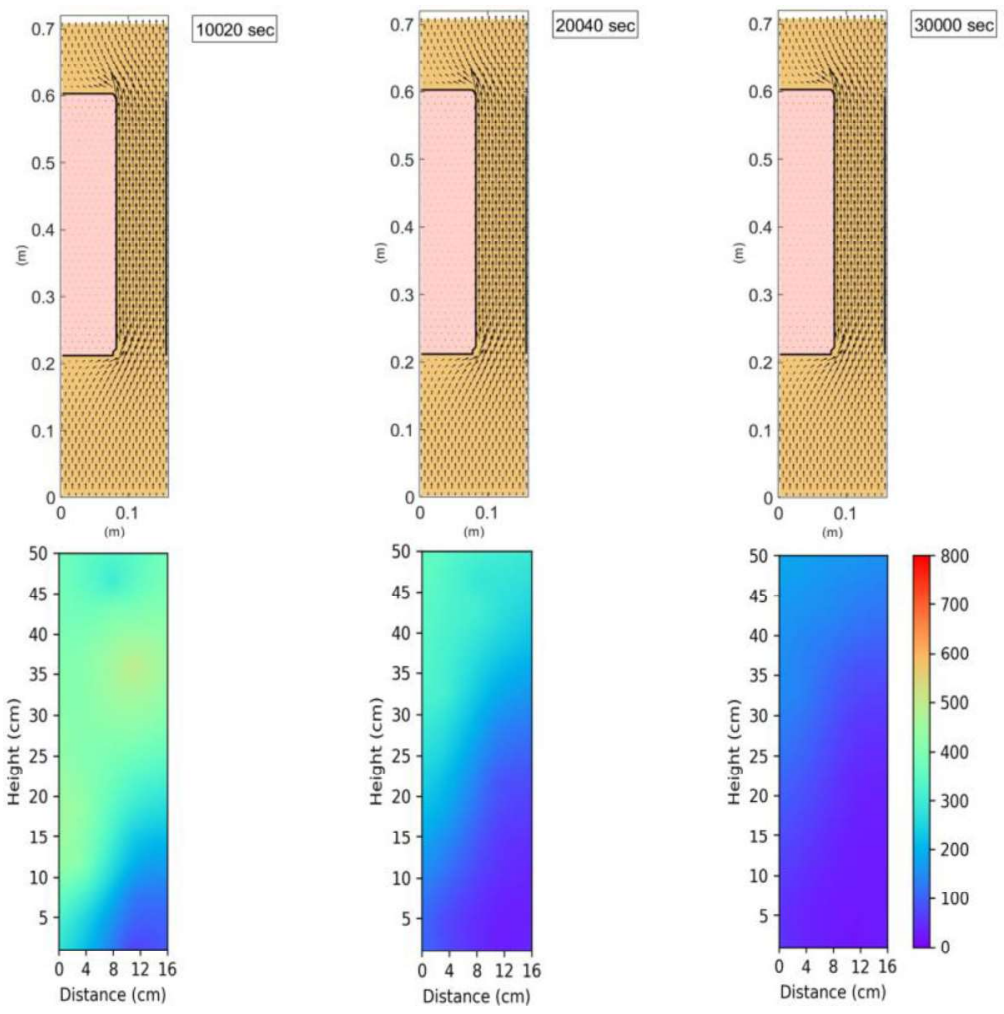

Figure G-2: Comparison of ISSM and Experimental results for Parallel 1 between 6030000 seconds. 

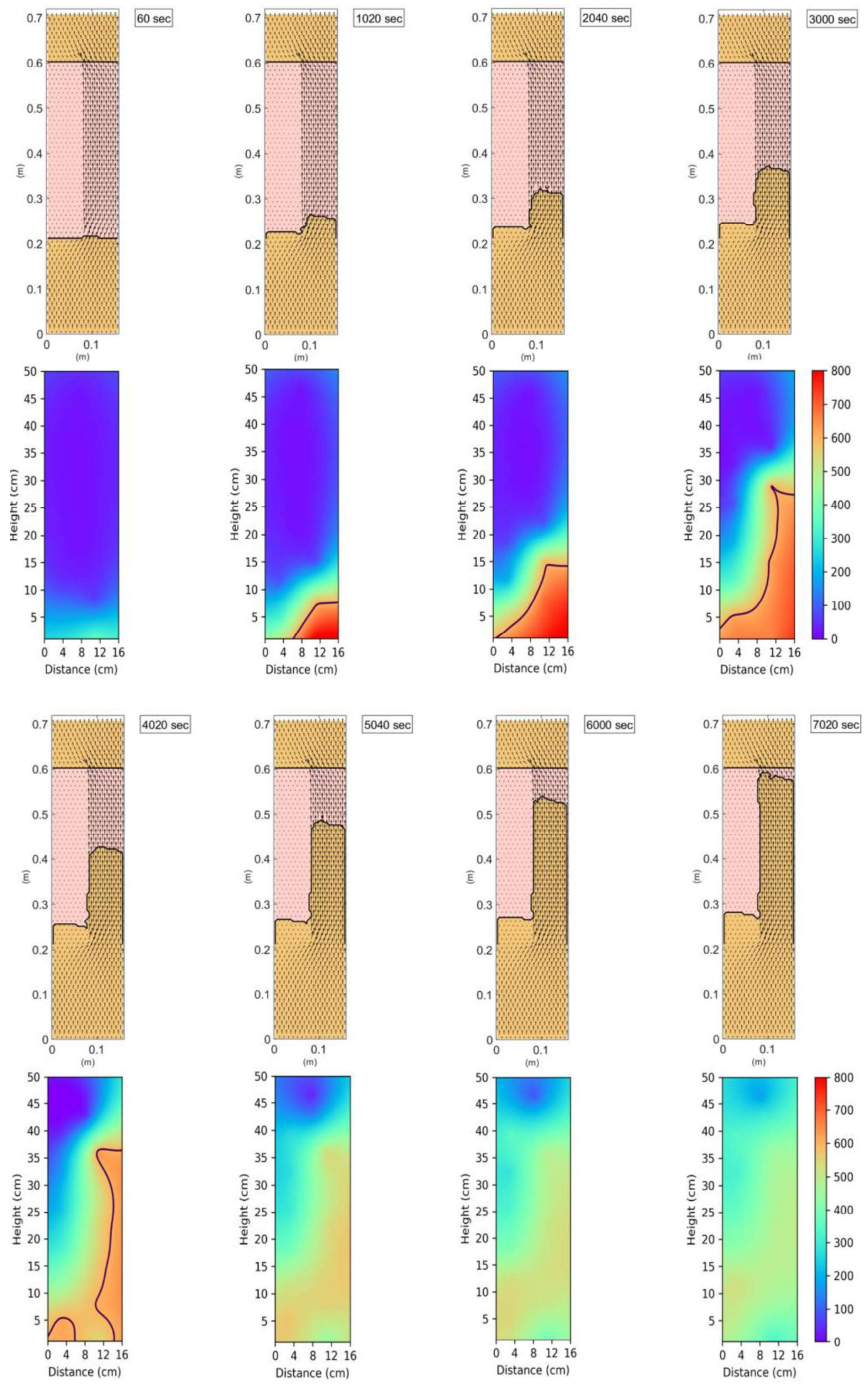

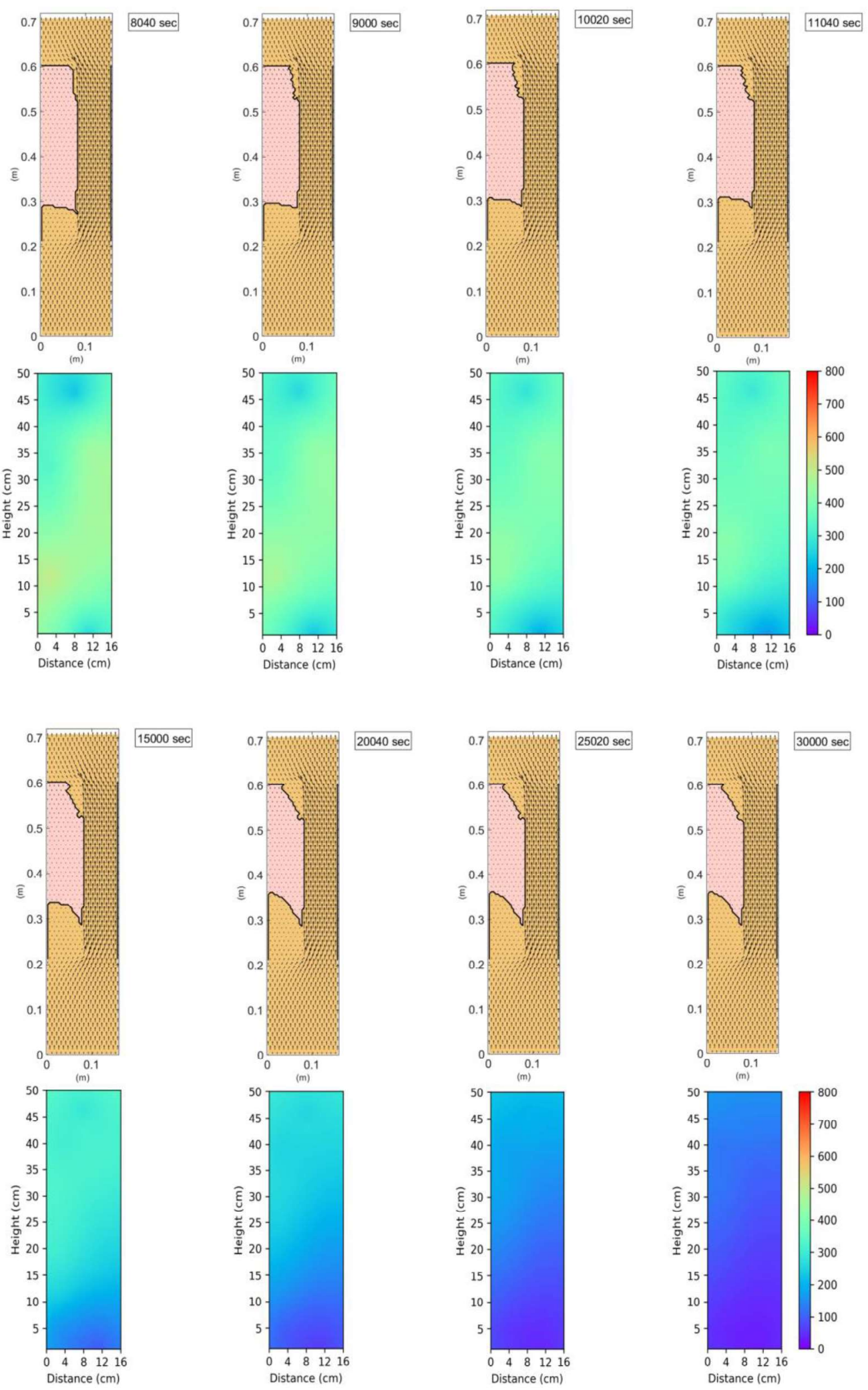

Figure G-3: Comparison of ISSM and Experimental results for Parallel 2 between 6030000 seconds. 

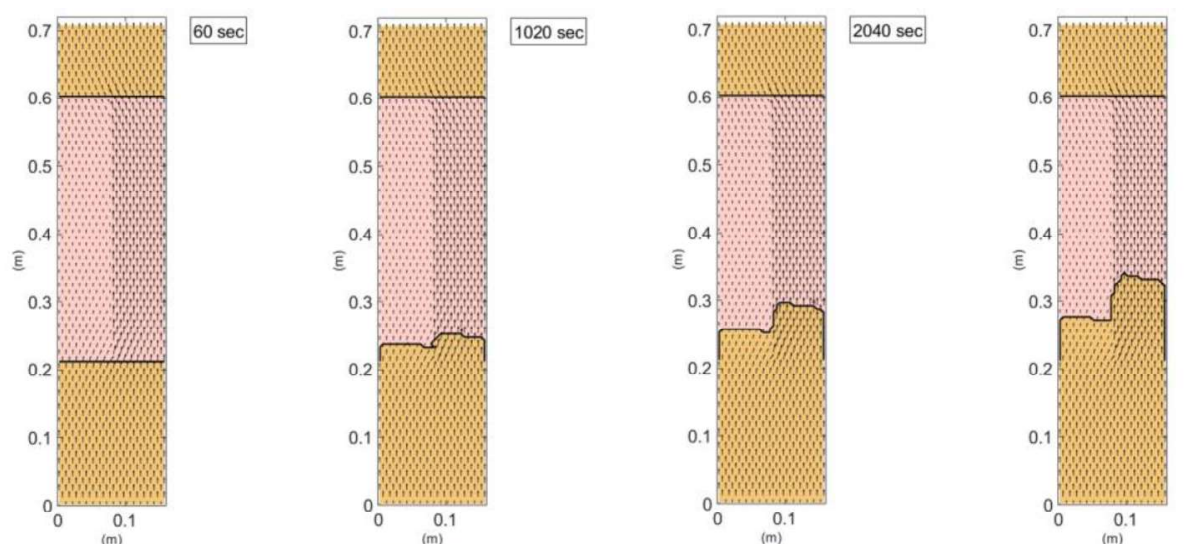

$3000 \mathrm{sec}$
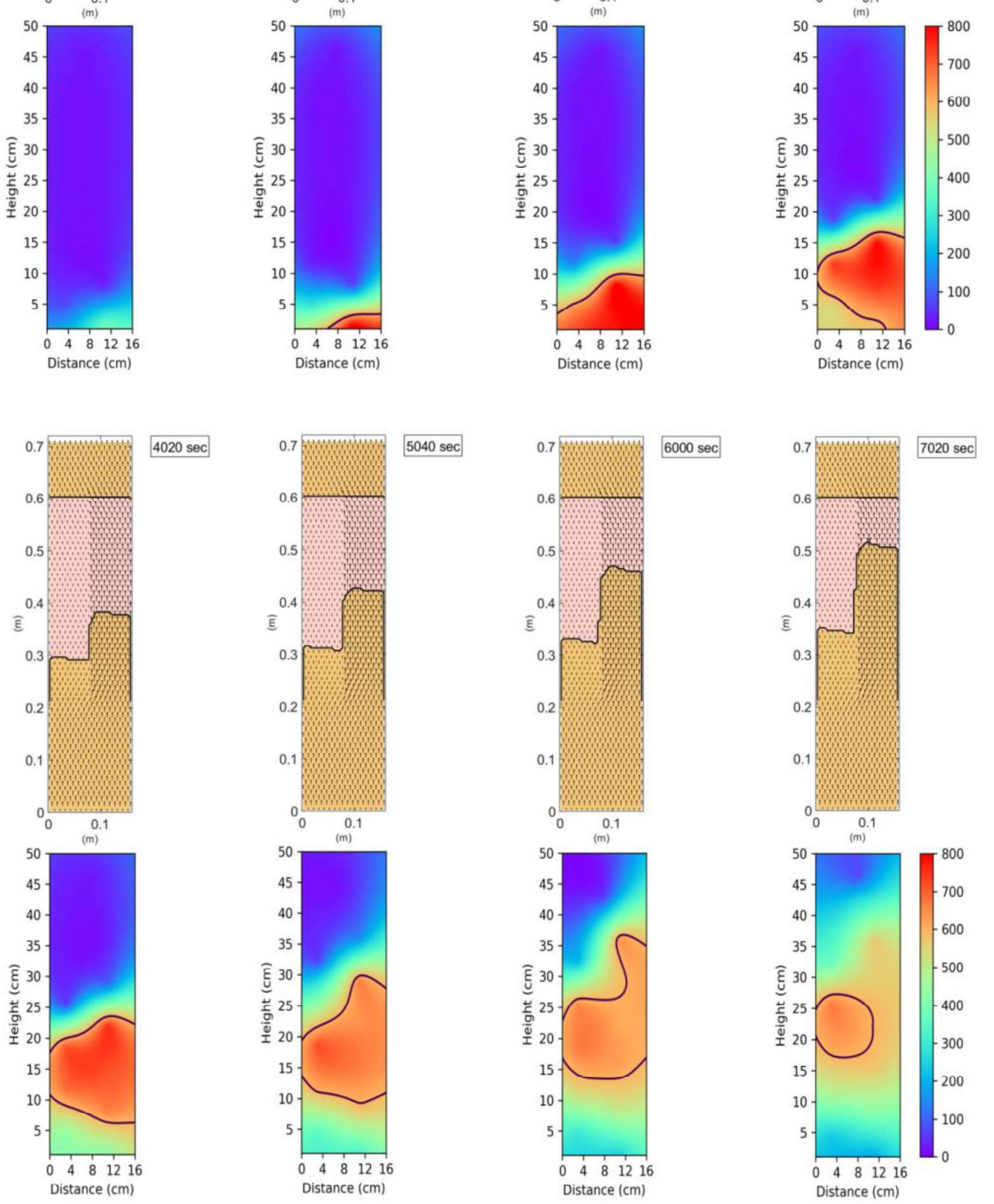

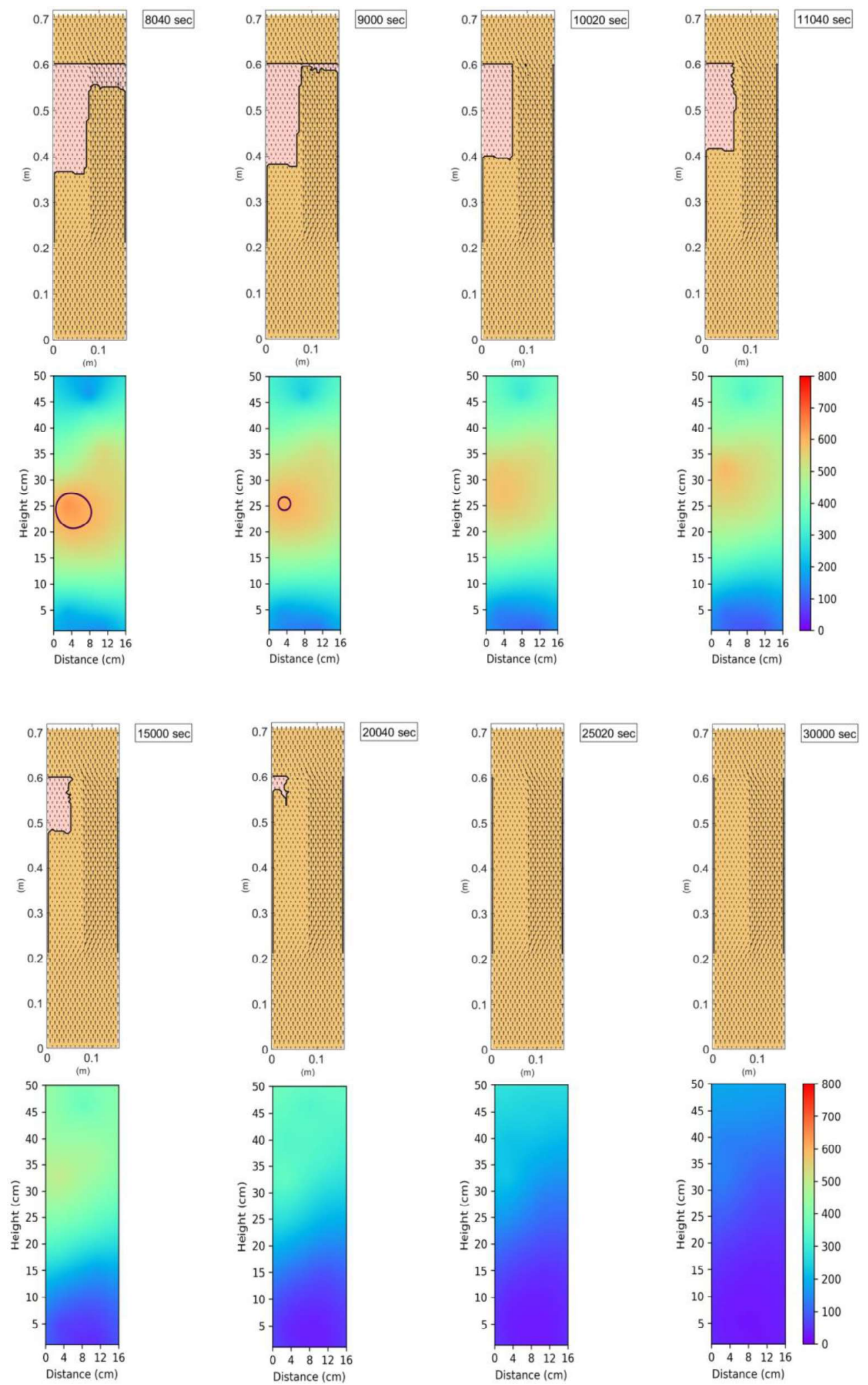

Figure G-4: Comparison of ISSM and Experimental results for Parallel 3 between 6030000 seconds. 

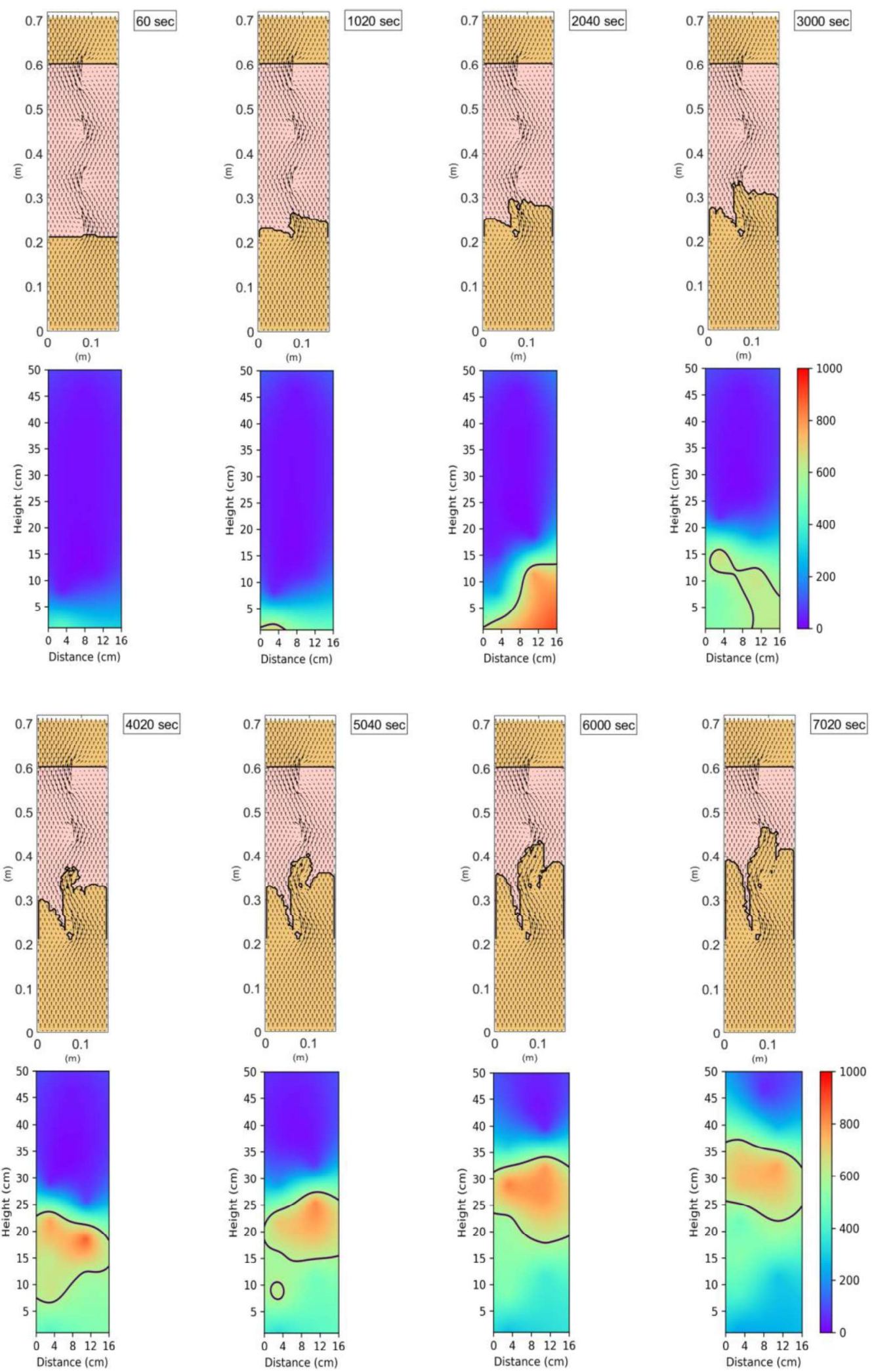

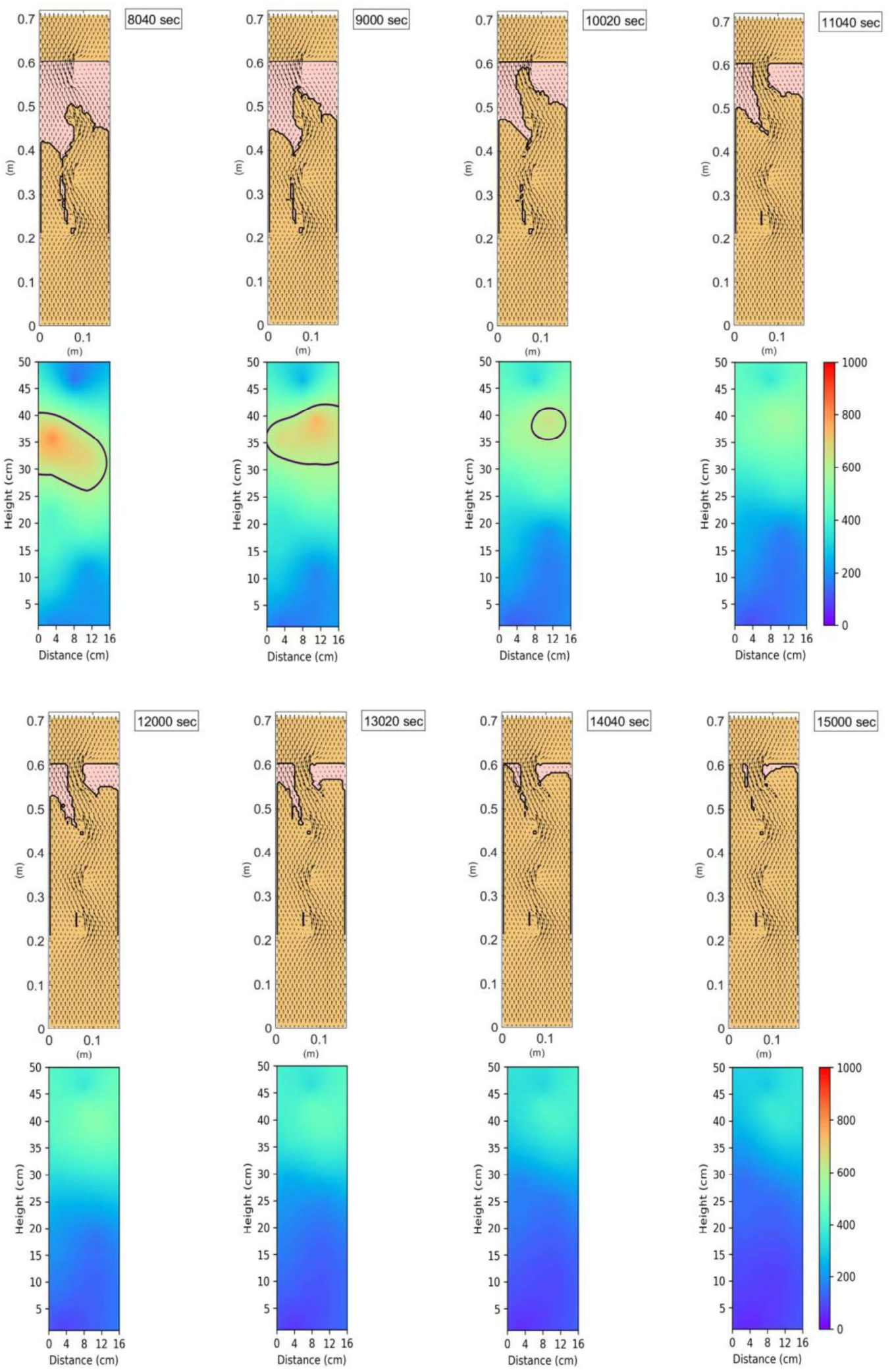

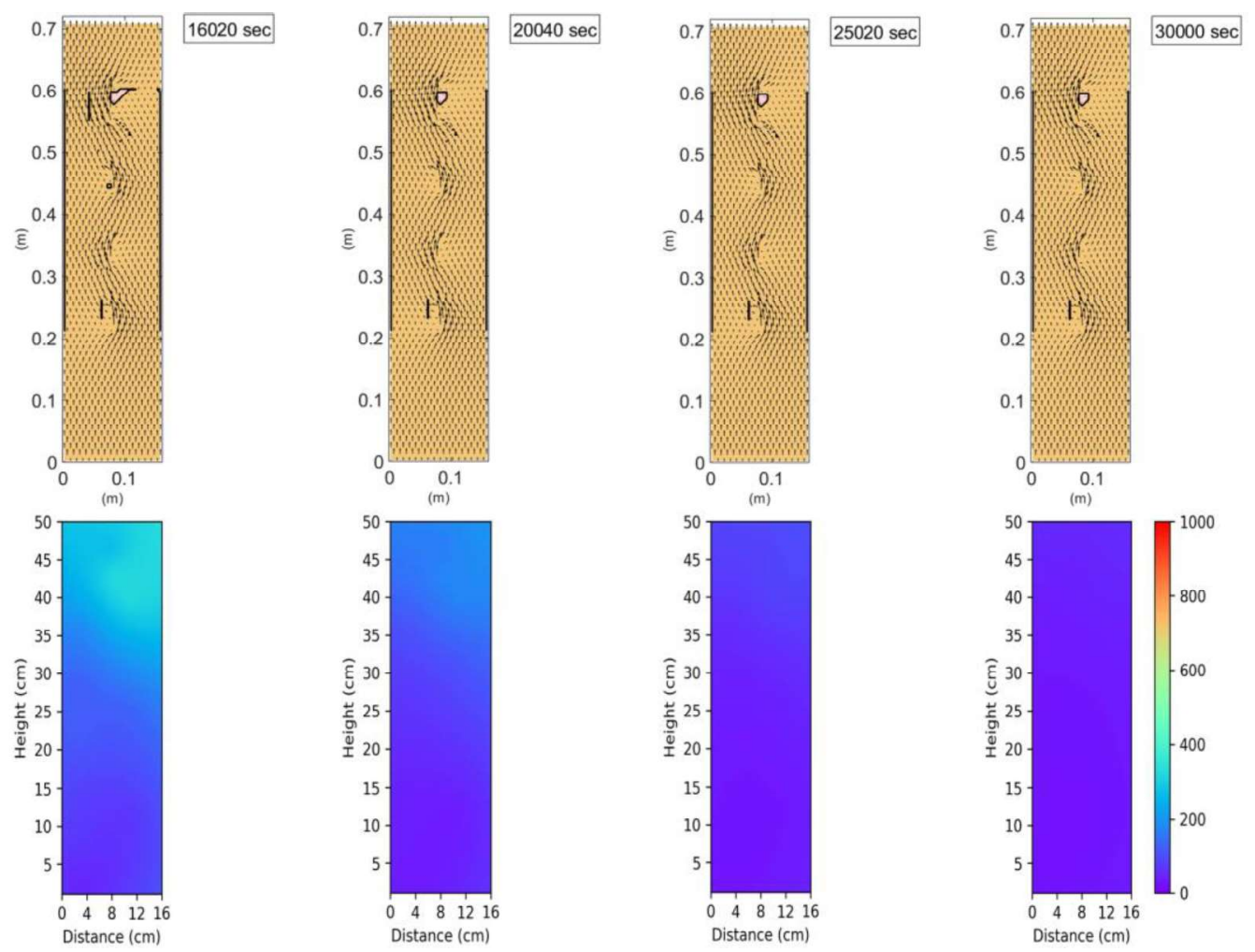

Figure G-5: Comparison of ISSM and Experimental results for Complex: Disconnected Coarse between 60-30000 seconds. 


\title{
Curriculum Vitae
}

\author{
Name: \\ Jiahao Wang \\ Post-secondary Beijing Forestry University \\ Education and Beijing, China \\ Degrees: $\quad$ 2013-2017 Bachelor of Engineering \\ The University of Western Ontario \\ London, Ontario, Canada \\ 2018-2020 Master of Engineering Science \\ Related Work Teaching Assistant \\ Experience The University of Western Ontario \\ 2018-2019 \\ Part-time Industrial Internship \\ Savron (a subdivision of Geosyntec Consultants) \\ 2018-2019 \\ Presenter \\ Symposium for New Research in Groundwater Science \\ 2019
}

